This work was supported by the US Department of Energy, Division of Geothermal and Hydropower Technologies.

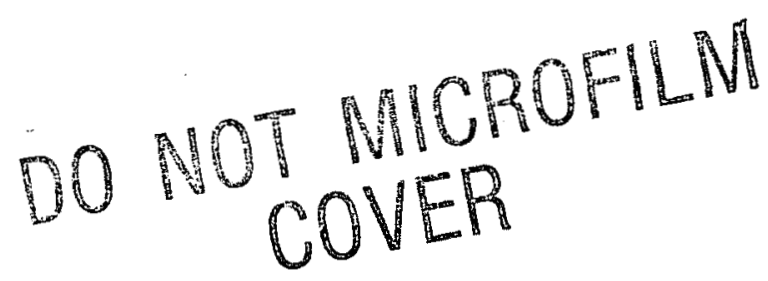

DISCLAIMER

This report was prepared as an account of work sponsored by an agency of the United States Government. Neither the United States Government nor any agency thereof, nor any of their employees, makes any warranty, express or implied, or assumes any legal liability or responsibility for the accuracy, completeness, or usefulness of any information, apparatus, product, or process disclosed, or represents that its use would not infringe privately owned rights. References herein to any specific commercial product, process, or service by trade name, trademark, manufacturer, or otherwise, does not necessarily constitute or imply its endorsement, recommendation, or favoring by the United States Government or any agency thereof. The views and opinions of authors expressed herein do not necessarily state or reflect those of the United States Government or any agency thereof. 


\section{DISCLAIMER}

This report was prepared as an account of work sponsored by an agency of the United States Government. Neither the United States Government nor any agency Thereof, nor any of their employees, makes any warranty, express or implied, or assumes any legal liability or responsibility for the accuracy, completeness, or usefulness of any information, apparatus, product, or process disclosed, or represents that its use would not infringe privately owned rights. Reference herein to any specific commercial product, process, or service by trade name, trademark, manufacturer, or otherwise does not necessarily constitute or imply its endorsement, recommendation, or favoring by the United States Government or any agency thereof. The views and opinions of authors expressed herein do not necessarily state or reflect those of the United States Government or any agency thereof. 


\section{DISCLAIMER}

Portions of this document may be illegible in electronic image products. Images are produced from the best available original document. 
LA - -9558-HDR

DE83 004480

\title{
Geothermal Investigations in West Virginia
}

\author{
Robert Hendry* \\ Kenneth Hilfiker* \\ Dennis Hodge* \\ Paul Morgan** \\ Chandler Swanberg*** \\ Spencer S. Shannon, Jr.
}

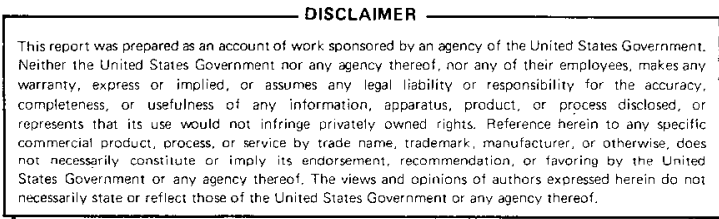

*Department of Geology, State University of New York/Buffalo, Amherst, NY 14226.

**Lunar and Planetary Institute, National Aeronautics and Space Administration, Houston,

TX 77058.

***Department of Geology, New Mexico State University, Las Cruces, NM 88003.

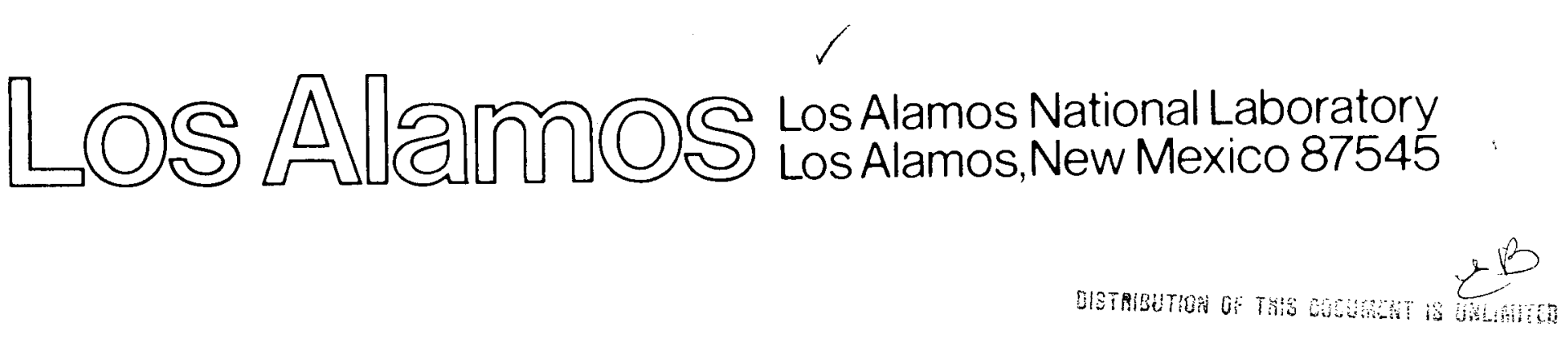




\title{
GEOTHERMAL INVESTIGATIONS IN WEST VIRGINIA
}

\author{
by \\ Robert Hendry, Kenneth Hilfiker, Dennis Hodge, Paul Morgan, Chandler \\ Swanberg, and Spencer S. Shannon, Jr.
}

\section{ABSTRACT}

Deep sedimentary basins and warm-spring systems in West Virginia are potential geothermal resources. A temperature gradient map based on 800 bottom-hole temperatures for West Virginia shows that variations of temperature gradient trend northeasterly, parallel to regional structure. Highest temperature gradient values of about $28^{\circ} \mathrm{C} / \mathrm{km}$ occur in east-central West virginia, and the lowest gradients $\left(18^{\circ} \mathrm{C} / \mathrm{km}\right)$ are found over the Rome Trough. Results from ground-water geochemistry indicate that the warm waters circulate in very shallow aquifers and are subject to seasonal temperature fluctuations. Silica heat-flow data in West Virginia vary from about 0.89 to $1.4 \mathrm{HFU}$ and generally increase towards the west. Bouguer, magnetic, and temperature gradient profiles suggest that an ancient rift transects the state and is the site of several deep sedimentary basins.

\section{INTRODUCTION}

Geothermal resources in eastern United States include (1) warm-spring systems, (2) radioactive granite plutons beneath thick sedimentary cover, and (3) deep sedimentary basins having normal temperature gradients. The Appalachian basin in West Virginia contains sedimentary rocks that are greater than $20000 \mathrm{ft}$ deep; thick sections of shale and sandstone occur in these regions. These deep basins are potentially attractive geothermal resources if higher-than-normal temperature gradients are identified. Numerous warm springs in eastern West virginia suggest that deep circulation of ground waters along faults may locally elevate wall rock temperatures in the Appalachian basin. This is a preliminary study of the potential geothermal 
resources and provides an analysis of the subsurface temperatures and heat flow of West Virginia.

II. REGIONAL GEOLOGY

For the most part, central West Virginia is located in the plateau region and is part of the Allegheny synclinorium. The rocks in the plateau region are not folded as much as the sedimentary rocks in the Valley and Ridge province to the east. Folding and thrust faulting are generaity restricted to the "thin skinned" sedimentary rocks. Geothermal signatures from basement rocks indicate that some structures may be rejuvenated older faults.

King and Zietz (1978) describe a prominent northeast-trending magnetic lineament nearly $1300 \mathrm{~km}$ long that is marked by a series of linear magnetic gradients that separate areas of contrasting magnetic rocks in the basement. The trend of this anomaly, called the New York-Alabama lineament, is subparallel to a major basement discontinuity which forms the eastern boundary of the Rome Trough in West Virginia. This magnetic lineament intersects a structural lineament, the 38th parallel lineament, in West Virginia near the Rome Trough. The New York-Alabama lineament delineates a major discontinuity in the crystalline basement that may be the result of intra-continental collision between the stable cratons of the continental interior and the more mobile Appalachian block of the continental margin during Grenville time (King and Zietz 1978). Sedimentary rocks in the Rome Trough thicken from approximately $7500 \mathrm{ft}(2300 \mathrm{~m})$ on the flanks to $20000 \mathrm{ft}(6000 \mathrm{~m})$ in the trough of this feature (Kulander and Dean 1978). Ammerman and Keller (1979) suggest that, the trough in Kentucky may have formed during periods of rifting in Avalonian time.

Major Appalachian deformation that is evident at the surface occurred from the middle Permian through the Triassic period and produced faulting and low-angle thrusting throughout the central plateau province and eastern west Virginia. Kulander and Dean (1978) suggest that reactivation of a major displacement in the crystalline basement took place during the Early Paleozoic era and continued into the Appalachian orogeny. Kulander and Dean (1978) noted that sedimentary lithologic facies change over short distances toward the margin of the Rome Trough and that normal faulting may have developed during Early Cambrian time.

The basement rocks throughout central West Virginia vary in lithology and are thought to be of Grenville age. Sample compositions from well cores range 
from granodiorite to anorthosite. Bass (1959) found granodiorite, tonalite, and bands of syenite, monzonite, and granite in basement core from Wood County, West Virginia. The basement rock from a well in Mingo County, West Virginia is anorthosite.

III. TEMPERATURE GRADIENT MAP OF WEST VIRGINIA

Hodge et a1. (1979) demonstrated in New York that bottom-hole temperatures from electric logs can describe lateral variation in geothermal gradients if statistical smoothing procedures are applied. To eliminate bottom-hole temperatures that may have been disturbed by pleistocene and annual variations in temperature or by shallow circulation of ground water, 792 wells (Appendix A) having depths greater than $500 \mathrm{~m}$ were used in geothermal-gradient calculations. Average surface temperatures at the National Oceanic and Atmospheric Administration (NOAA) recording stations for the surrounding eight states were used to develop the mathematical estimate of surface temperatures at sea level throughout West Virginia. Temperatures at elevations of each well were calculated using a lapse rate of $9.8^{\circ} \mathrm{C} / \mathrm{km}$. Geothermal gradients were then calculated by subtracting surface temperatures from bottom-hole temperatures and dividing by the depths. No corrections for drilling disturbance have been applied to these data. In this statistical contouring approach, randomly scattered data having geothermal gradients of 6.7 to $61.2^{\circ} \mathrm{C} / \mathrm{km}$ are replaced by a regularly spaced grid matrix having gradients of 11.9 to $37.1^{\circ} \mathrm{C} / \mathrm{km}$. Subsequently, these data were smoothed resulting in gradients between 16.4 and $33.3^{\circ} \mathrm{C} / \mathrm{km}$.

The geothermal gradient map of West Virginia (Fig. 1) has a relatively smooth pattern with the temperature gradients trending northeasterly. The highest values are in the east-central part of the state, and a prominent low coincides with the Rome Trough in the south-central area.

\section{GEOCHEMISTRY}

Ground-water geochemistry, which is the primary source of information for this phase of the investigation, can be used to determine regional heat flow and to delineate specific areas having high-geothermal potential. Similar studies in New York have been useful in delineating potential hot, dry rock geothermal resources (Hilfiker et al. 1980). This report emphasizes three aspects of geochemical reconnaissance: 


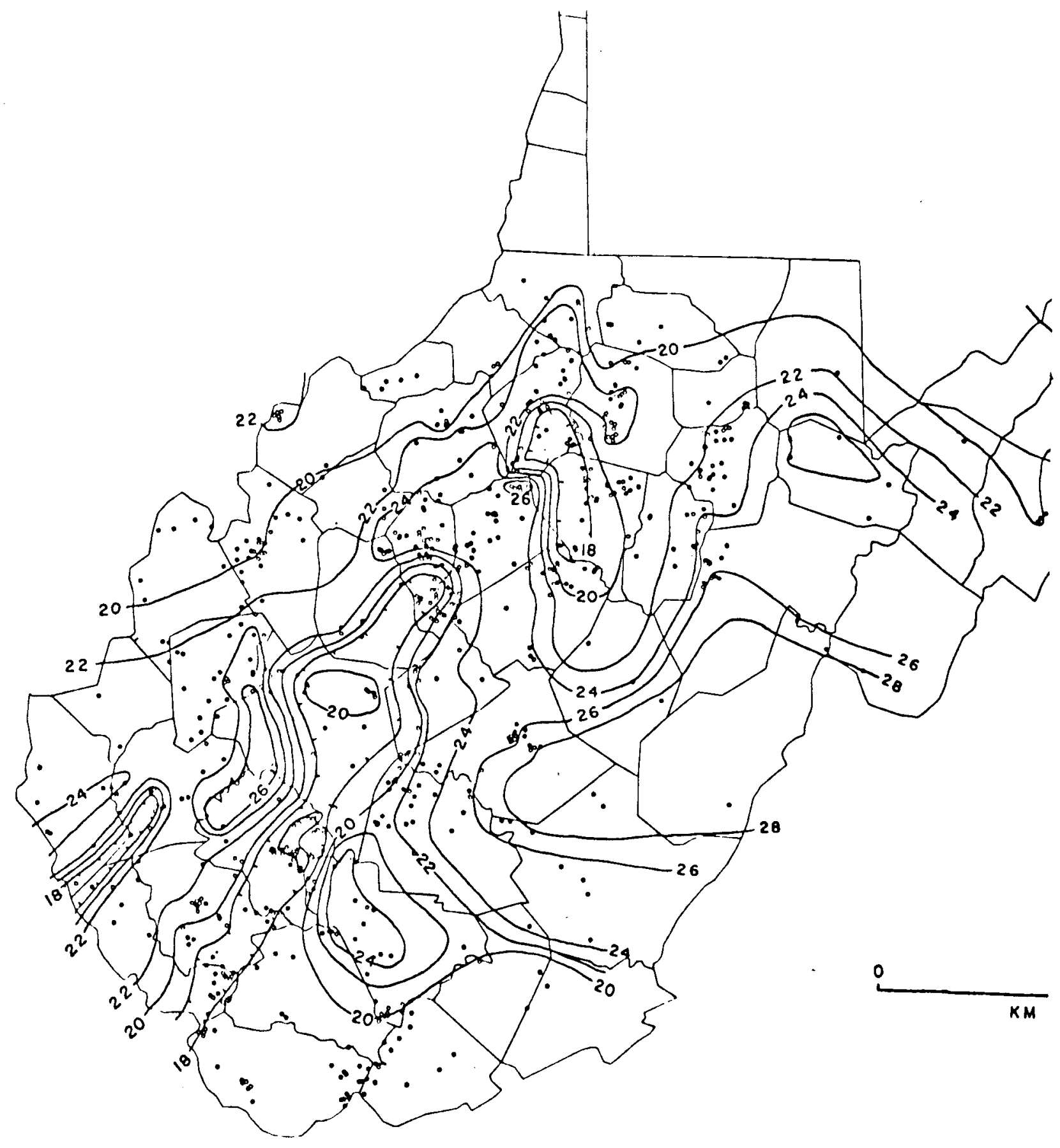

Fig. 1.

Geothermal gradient map of West Virginia. The small circles are bottom-hole temperature locations, and the contour interval is $2^{\circ} \mathrm{C} / \mathrm{km}$. The gradients have not been corrected for drilling disturbance. 
1. By using routine chemical analyses of hot-spring waters and by applying the silica and NaKCa geothermometers (Fournier and Rowe 1966; Fournier and Truesdell 1973), one can determine if these waters originated within an active geothermal system and can estimate the temperatures within the geothermal reservoir.

2. The same geothermometers can be applied to chemical analyses of nonthermal ground water (Swanberg and Alexander 1979). Although waters which originated in geothermal reservoirs may cool during circulation before arriving at a surface discharge point, they maintain chemical characteristics compatible with their thermal origin. Thus waters of thermal origin can be detected even though they discharge as cold springs or are collected from cold wells.

3. Based on the observed empirical relation between temperatures estimated by the silica geothermometer and regional heat flow (Swanberg and Morgan 1978/1979; 1980), the silica content of ground waters can be used quantitatively to estimate regional heat flow and thereby predict the thermal regime for any geologic environment for which ground-water silica data are available.

The evaluation of approximately 500 chemical analyses is described in the following sections.

Hot Springs Study. Most of the 30 hot springs in West Virginia (Waring 1965) are in the Valley and Ridge province in the eastern part of the state. Perry and Costain (1979) report the following mechanism for the origin of nearby hot springs in the Valley and Ridge province in Virginia: Meteoric waters enter near-vertical sedimentary beds at relatively high topographic elevations and move downward along permeable bedding planes and fractures. This moderately deep circulation intersects vertical, transverse linear fracture zones through which the water rises to the surface. Furthermore, warm springs are adjacent to topographic gaps which have developed along zones of weakness associated with the fracture zones. A similar mechanism could explain the origin of hot springs in the Valley and Ridge province in West Virginia. Geochemical data support this model for the Virginia hot springs, but not for the West Virginia ones.

About two-thirds of the hot springs catalogued by waring (1965) were sampled in this study. In addition, data from Berkeley and Minnehaha Springs were taken from Hobba et al. (1977). Most temperatures we measured ( 7 to 
$15^{\circ} \mathrm{C}$, Table I) are significantly lower than the temperatures reported by Waring $\left(1965 ; 16\right.$ to $\left.23^{\circ} \mathrm{C}\right)$. Either we failed to locate the true spring, or we may not have tested the actual discharge point. However, a few reliable temperatures were obtained from exactly the same discharge points reported by Waring (1965); these springs simply are not thermal. Thus many "hot" springs in West Virginia seem to circulate in shallow aquifers, possibly reaching the surface where they undergo large seasonal temperature fluctuations. This conclusion is supported by the chemical geothermometers. The average temperature estimated by the silica geothermometer for the "hot" springs is $30.8^{\circ} \mathrm{C}$, which is lower than the average temperature for West Virginia ground waters $\left(41.5^{\circ} \mathrm{C}\right)$ and is, in fact, closer to the mean value for surface waters $\left(23.4^{\circ} \mathrm{C}\right)$. Using the NaKCa geothermometer, most "hot" springs have negative temperatures which indicate that the waters' residence time in the ground has been too short to reach chemical equilibrium with the host rock.

The above conclusions do not apply to the Berkeley or Minnehaha Springs in West Virginia, which are hotter and yield realistic temperature estimates from use of the silica and NaKCa geothermometers. Thus the deep circulation model of Perry and Costain (1979) may well apply to these few springs.

Heat Flow. The silica heat-flow technique of Swanberg and Morgan $(1978 / 1979 ; 1980)$ is designed to convert ground-water silica data to regional heat flow. This method can be used to supplement traditional heat-flow data by more precisely determining heat-flow trends, specific heat-flow anomalies, and other features that relate to the location and evaluation of hot, dry rock geothermal resources. In this study, we have used about 500 ground-water silica analyses from the US Geological Survey WATSTORE file and the published literature. Also, 44 additional samples were collected and analyzed for silica to fill gaps in the published data. The analyses are listed in Tables II and III. The ground-water data have been converted to heat-flow values by using the equations of Swanberg and Morgan (1980).

The silica heat-flow data have been grouped geographically in several ways to correlate silica heat flow with other geological and geophysical data. Silica heat flow tends to increase westward. The lowest values are to the east in the Valley and Ridge province where the mean silica heat flow is 0.95 heat-flow units (HFU). Four traditional heat-flow values reported by Perry and Costain (1979) for the Valley and Ridge-Blue Ridge province of nearby Virginia average $1.06 \mathrm{HFU}$, which is in good agreement with the silica data. 
TABLE I

WEST VIRGINIA DATA

SIT, CHT, and CCHT are temperatures estimated by the silica, NaKCa, and $\mathrm{MaKCa} \mathrm{Mg}$ geothermometers, respectively.

\begin{tabular}{|c|c|c|c|c|c|c|c|}
\hline \multirow{2}{*}{$\begin{array}{l}\text { Sample } \\
\text { No. }\end{array}$} & \multirow[b]{2}{*}{ Name } & \multicolumn{2}{|c|}{ Location } & \multirow{2}{*}{$\begin{array}{l}\text { Temp. } \\
{ }^{\circ} \mathrm{C}\end{array}$} & \multirow{2}{*}{$\begin{array}{l}\text { SIT } \\
{ }^{\circ} \mathrm{C}\end{array}$} & \multirow{2}{*}{$\begin{array}{l}\mathrm{CHT} \\
{ }^{\circ} \mathrm{C}\end{array}$} & \multirow{2}{*}{${ }^{\mathrm{C}} \mathrm{CHT}$} \\
\hline & & Lat. & Long. & & & & \\
\hline 1 & Piercy's Cave Spring & $37^{\circ} 50.8^{\prime}$ & $80^{\circ} 34.8^{\prime}$ & 9.0 & 28.5 & -5.8 & -5.8 \\
\hline 2 & Piercy's Cave Spring & $37^{\circ} 51.0^{\prime}$ & $80^{\circ} 34.5^{\prime}$ & 6.0 & 28.9 & 18.1 & 18.1 \\
\hline 3 & Piercy's Cave Spring & $37^{\circ} 50.0^{\prime}$ & $80^{\circ} 34.9^{\prime}$ & 10.0 & 28.9 & -5.5 & -5.5 \\
\hline 4 & Sweet Calybeate Spring & $37^{\circ} 38.8^{\prime}$ & $80^{\circ} 14.2^{\prime}$ & 22.0 & 51.3 & 53.1 & 53.1 \\
\hline 5 & old Sweet Springs & $37^{\circ} 37.6^{\prime}$ & $80^{\circ} 14.0^{\prime}$ & 7.0 & 27.6 & -19.2 & -19.2 \\
\hline 6 & Boiling Spring & $37^{\circ} 41.8^{\prime}$ & $80^{\circ} 06.1^{\prime}$ & 11.0 & 28.5 & -8.8 & -8.8 \\
\hline 7 & Salt Sulphur Spring & $37^{\circ} 33.9^{\prime}$ & $80^{\circ} 34.1^{\prime}$ & - & 56.0 & 3.1 & 3.1 \\
\hline 8 & Iodine Spring & $37^{\circ} 34.1^{\prime}$ & $80^{\circ} 34.0^{\prime}$ & - & 32.5 & 4.3 & 4.3 \\
\hline 9 & Ewing Run Cold Spring & $37^{\circ} 31.4^{\prime}$ & $80^{\circ} 18.1^{\prime}$ & 7.0 & 22.4 & -22.2 & -22.2 \\
\hline 10 & Hot Springs & $38^{\circ} 00.1^{\prime}$ & $79^{\circ} 50.1^{\prime}$ & 32.0 & 56.0 & 56.2 & 56.2 \\
\hline 11 & Warm Spring & $38^{\circ} 03.0^{\prime}$ & $79^{\circ} 47.8^{\prime}$ & 36.0 & 68.7 & 37.6 & 37.6 \\
\hline 12 & Ruckman Run & $38^{\circ} 12.3^{\prime}$ & $79^{\circ} 57.1^{\prime}$ & 10.0 & 19.8 & -18.6 & -18.6 \\
\hline 13 & Guy Run Springs & $38^{\circ} 13.3^{\prime}$ & $79^{\circ} 55.9^{\prime}$ & - & 13.9 & -13.4 & -13.4 \\
\hline 14 & Mill Run Spring & $38^{\circ} 14.3^{\prime}$ & $79^{\circ} 55.0^{\prime}$ & 13.0 & 30.6 & -12.4 & -12.4 \\
\hline 15 & Dunmore Drinking Spring & $38^{\circ} 21.5^{\prime}$ & $79^{\circ} 52.5^{\prime}$ & 15.0 & 36.2 & -5.5 & -5.5 \\
\hline 16 & S.P. Curry Spring & $38^{\circ} 11.1^{\prime}$ & $80^{\circ} 01.0^{\prime}$ & 8.0 & 22.9 & -29.7 & -29.7 \\
\hline 17 & White Sulphur Spring & $37^{\circ} 47.8^{\prime}$ & $80^{\circ} 17.9^{\prime}$ & 12.0 & 52.8 & -1.6 & -1.6 \\
\hline 18 & Cress Spring & $38^{\circ} 37.5^{\prime}$ & $79^{\circ} 19.9^{\prime}$ & 8.0 & 19.8 & -15.7 & -15.7 \\
\hline 19 & Roaring Spring at Circleville & $38^{\circ} 40.8^{\prime}$ & $79^{\circ} 28.9^{\prime}$ & 4.0 & 15.1 & 7.3 & 7.3 \\
\hline 20 & Big Spring & $38^{\circ} 55.6^{\prime}$ & $79^{\circ} 31.2^{\prime}$ & 8.0 & 19.8 & -17.0 & -17.0 \\
\hline 21 & & $38^{\circ} 56.9^{\prime}$ & $79^{\circ} 17.5^{\prime}$ & 10.0 & 18.1 & -7.3 & -7.3 \\
\hline 22 & Shannondale Spring & $39^{\circ} 13.0^{\prime}$ & $77^{\circ} 48.8^{\prime}$ & - & 50.3 & 37.2 & 37.2 \\
\hline 23 & Mountain Mission & $39^{\circ} 17.3^{\prime}$ & $77^{\circ} 46.2^{\prime}$ & 11.0 & 42.7 & 22.1 & 22.1 \\
\hline 24 & Swan Pond Spring & $39^{\circ} 28.0^{\prime}$ & $77^{\circ} 58.2^{\prime}$ & - & 49.3 & -4.1 & $-4 \cdot 1$ \\
\hline 25 & Cold Stream Run & $39^{\circ} 20.0^{\prime}$ & $78^{\circ} 27.1^{\prime}$ & 11.0 & 30.1 & -16.2 & -16.2 \\
\hline 26 & Capon Springs & $39^{\circ} 08.2^{\prime}$ & $78^{\circ} 28.9^{\prime}$ & - & 36.9 & -16.0 & -16.0 \\
\hline 28 & Warm (Boiling) Spring & $39^{\circ} 01.7^{\prime}$ & $78^{\circ} 36.5$ & 14.0 & 36.9 & -12.3 & -12.3 \\
\hline 29 & Terra Alta & $39^{\circ} 25.1^{\prime}$ & $79^{\circ} 33.1^{\prime}$ & 11.0 & 22.4 & 28.2 & 28.2 \\
\hline 145 & & $38^{\circ} 40.8^{\prime}$ & $80^{\circ} 52.9^{\prime}$ & - & 40.2 & 33.6 & 33.6 \\
\hline 146 & & $38^{\circ} 34.8^{\prime}$ & $80^{\circ} 55.9^{\prime}$ & - & 47.8 & 29.2 & 29.2 \\
\hline 147 & · & $38^{\circ} 50.3^{\prime}$ & $80^{\circ} 59.0^{\prime}$ & - & 33.3 & 62.6 & 62.6 \\
\hline 148 & & $38^{\circ} 46.1^{\prime}$ & $81^{\circ} 06.4^{\prime}$ & - & 44.7 & 15.4 & 15.4 \\
\hline 149 & & $38^{\circ} 40.2^{\prime}$ & $81^{\circ} 05.0^{\prime}$ & - & 41.5 & 48.8 & 48.8 \\
\hline 150 & & $38^{\circ} 33.1^{\prime}$ & $81^{\circ} 01.5^{\prime}$ & - & 33.7 & 72.8 & 47.0 \\
\hline 151 & & $38^{\circ} 29.1^{\prime}$ & $81^{\circ} 09.1^{\prime}$ & - & 36.6 & 31.8 & 31.8 \\
\hline 152 & & $38^{\circ} 29.1^{\prime}$ & $81^{\circ} 09.1^{\prime}$ & - & 43.3 & 44.5 & 44.5 \\
\hline 153 & & $38^{\circ} 32.5^{\prime}$ & $81^{\circ} 20.8^{\prime}$ & - & 43.6 & 38.8 & 38.8 \\
\hline 154 & & $38^{\prime} 37.9^{\prime}$ & $81^{\circ} 23.6^{\prime}$ & - & 49.3 & 7.3 & 7.3 \\
\hline 155 & & $38^{\circ} 38.5^{\prime}$ & $81^{\circ} 21.2^{\prime}$ & 13.0 & 37.2 & 51.1 & 51.1 \\
\hline
\end{tabular}


TABLE I (cont.)

\begin{tabular}{|c|c|c|c|c|c|c|c|}
\hline \multirow{2}{*}{$\begin{array}{l}\text { Sample } \\
\text { No. }\end{array}$} & \multirow[b]{2}{*}{ Name } & \multicolumn{2}{|c|}{ Location } & \multirow{2}{*}{$\begin{array}{l}\text { Temp. } \\
{ }^{\circ} \mathrm{C}\end{array}$} & \multirow{2}{*}{${ }^{\circ} \mathrm{CIT}$} & \multirow{2}{*}{$\begin{array}{l}\mathrm{CHT} \\
{ }^{\circ} \mathrm{C}\end{array}$} & \multirow{2}{*}{$\begin{array}{l}\mathrm{CCHT} \\
{ }^{\circ} \mathrm{C}\end{array}$} \\
\hline & & Lat. & Long. & & & & \\
\hline 156 & & $38^{\circ} 43.4^{\prime}$ & $81^{\circ} 23.9^{\prime}$ & 14.0 & 35.1 & 10.9 & 10.9 \\
\hline 157 & & $38^{\circ} 48.0^{\prime}$ & $81^{\circ} 24.5^{\prime}$ & - & 41.2 & 53.0 & 53.0 \\
\hline 158 & & $38^{\circ} 44.0^{\prime}$ & $81^{\circ} 17.1^{\prime}$ & - & 63.1 & -11.4 & -11.4 \\
\hline 159 & & $38^{\circ} 37.3^{\prime}$ & $81^{\circ} 13.1^{\prime}$ & - & 36.9 & 60.7 & 60.7 \\
\hline 160 & & $38^{\circ} 24.5^{\prime}$ & $81^{\circ} 06.9^{\prime}$ & - & 48.3 & 26.8 & 26.8 \\
\hline 161 & & $38^{\circ} 19.3^{\prime}$ & $81^{\circ} 10.2^{\prime}$ & - & 32.5 & 32.0 & 32.0 \\
\hline 162 & & $39^{\circ} 03.0^{\prime}$ & $80^{\circ} 32.0^{\prime}$ & - & 34.4 & 48.7 & 48.7 \\
\hline 163 & & $39^{\circ} 02.6^{\prime}$ & $80^{\circ} 37.3^{\prime}$ & - & 37.2 & 61.5 & 61.5 \\
\hline 164 & & $39^{\circ} 02.6^{\prime}$ & $80^{\circ} 37.3^{\prime}$ & 19.0 & 32.9 & 10.3 & 10.3 \\
\hline 165 & & $39^{\circ} 00.8^{\prime}$ & $80^{\circ} 43.6^{\prime}$ & - & 44.4 & 42.4 & 42.4 \\
\hline 166 & & $39^{\circ} 02.8^{\prime}$ & $80^{\circ} 49.5^{\prime}$ & - & 46.1 & 21.6 & 21.6 \\
\hline 167 & & $39^{\circ} 02.8^{\prime}$ & $80^{\circ} 57.5^{\prime}$ & - & 56.6 & 12.0 & 12.0 \\
\hline 168 & & $39^{\circ} 05.0^{\prime}$ & $81^{\circ} 08.2^{\prime}$ & - & 51.3 & 26.8 & 26.8 \\
\hline 169 & & $39^{\circ} 08.6^{\prime}$ & $81^{\circ} 02.9^{\prime}$ & - & 36.6 & 73.5 & 73.5 \\
\hline 170 & & $39^{\circ} 15.2^{\prime}$ & $81^{\circ} 02.9^{\prime}$ & - & 38.9 & 20.9 & 20.9 \\
\hline 171 & & $39^{\circ} 16.5^{\prime}$ & $80^{\circ} 55.2^{\prime}$ & - & 50.8 & 47.1 & 47.1 \\
\hline 172 & & $39^{\circ} 17.5^{\prime}$ & $80^{\circ} 47.6^{\prime}$ & - & 56.6 & 17.6 & 17.6 \\
\hline 173 & & $39^{\circ} 16.9^{\prime}$ & $80^{\circ} 48.3^{\prime}$ & - & 37.2 & 50.6 & 50.6 \\
\hline 174 & & $39^{\circ} 16.8^{\prime}$ & $80^{\circ} 41.5^{\prime}$ & - & 53.7 & - & - \\
\hline 175 & & $39^{\circ} 16.1^{\prime}$ & $80^{\circ} 32.4^{\prime}$ & - & 45.0 & 51.3 & 51.3 \\
\hline 176 & & $39^{\circ} 11.9^{\prime}$ & $80^{\circ} 32.4^{\prime}$ & - & 45.0 & 51.3 & 51.3 \\
\hline 177 & & $39^{\circ} 11.2^{\prime}$ & $80^{\circ} 38.6^{\prime}$ & - & 55.3 & 9.5 & 9.5 \\
\hline 178 & & $39^{\circ} 11.9^{\prime}$ & $80^{\circ} 43.1^{\prime}$ & - & 52.3 & 31.4 & 31.4 \\
\hline 179 & & $39^{\circ} 22.7^{\prime}$ & $80^{\circ} 52.5^{\prime}$ & - & 40.2 & 65.5 & 65.5 \\
\hline 180 & & $39^{\circ} 27.5^{\prime}$ & $80^{\circ} 49.8^{\prime}$ & - & 68.0 & 1.5 & 1.5 \\
\hline 181 & & $39^{\circ} 31.3^{\prime}$ & $80^{\circ} 53.7^{\prime}$ & - & 39.2 & 22.8 & 22.8 \\
\hline 182 & & $39^{\circ} 34.7^{\prime}$ & $80^{\circ} 44.2^{\prime}$ & - & 55.3 & 9.6 & 9.6 \\
\hline 183 & & $39^{\circ} 30.4^{\prime}$ & $80^{\circ} 34.8^{\prime}$ & - & 38.3 & 2.3 & 2.3 \\
\hline 184 & & $39^{\circ} 23.8^{\prime}$ & $80^{\circ} 29.6^{\prime}$ & - & 22.9 & 22.0 & 22.0 \\
\hline 185 & & $39^{\circ} 35.5^{\prime}$ & $80^{\circ} 22.4^{\prime}$ & - & 55.1 & 25.3 & 25.3 \\
\hline 186 & & $39^{\circ} 38.0^{\prime}$ & $80^{\circ} 33.6^{\prime}$ & - & 34.8 & 24.4 & 24.4 \\
\hline 187 & & $39^{\circ} 40.5^{\prime}$ & $80^{\circ} 27.8^{\prime}$ & - & 46.1 & 21.0 & 21.0 \\
\hline 188 & & $39^{\circ} 36.5^{\prime}$ & $80^{\circ} 39.6^{\prime}$ & - & 40.5 & 31.1 & 31.1 \\
\hline 189 & & $39^{\circ} 43.0^{\prime}$ & $80^{\circ} 32.1$ & - & 45.0 & 18.5 & 18.5 \\
\hline 190 & & $39^{\circ} 48.0^{\prime}$ & $80^{\circ} 33.3^{\prime}$ & - & 42.1 & 18.6 & 18.6 \\
\hline 301 & Berkeley Springs & $39^{\circ} 37.6^{\prime}$ & $78^{\circ} 13.8^{\prime}$ & 22.2 & 37.9 & -3.9 & -3.9 \\
\hline 401 & Minnehaha Spring & $38^{\circ}-09.8^{\prime}$ & $79^{\circ} 58.5^{\prime}$ & 20.1 & 34.0 & -10.1 & -10.1 \\
\hline
\end{tabular}


TABLE II

CHEMICAL ANALYSIS OF SPRINGS AND WELLS IN WEST VIRGINIA, mg/ $/$

\begin{tabular}{|c|c|c|c|c|c|c|c|c|c|c|c|c|}
\hline $\begin{array}{c}\text { Sample } \\
\text { No. }\end{array}$ & $\mathrm{Ca}$ & $\mathrm{Mg}$ & $\mathrm{Na}$ & $k$ & $\mathrm{Cl}$ & $\mathrm{CO}_{3}$ & $\mathrm{HCO}_{3}$ & $\mathrm{SO}_{4}$ & $\mathrm{Fe}$ & B & SiO & $\mathrm{F}$ \\
\hline 1 & 40.3 & 0.2 & 1.4 & 1.2 & 5.7 & 0 & 125.7 & 5.8 & 0.10 & 0.09 & 7.00 & 0.20 \\
\hline 2 & 9.4 & 1.7 & 2.5 & 1.2 & 3.5 & 0 & 35.4 & 6.7 & 0.10 & 0.01 & 7.10 & 0.20 \\
\hline 3 & 39.3 & 0.2 & 1.4 & 1.2 & 5.7 & 0 & 124.5 & 7.2 & 0.10 & 0.02 & 7.10 & 0.20 \\
\hline 4 & 313.6 & 1.2 & 19.8 & 25.4 & 20.6 & 0 & 306.3 & 527.8 & 0.32 & 0.11 & 14.20 & 0.83 \\
\hline 5 & 47.5 & 0.1 & 0.7 & 0.8 & 3.5 & 0 & 137.9 & 9.1 & 0.10 & 0.01 & 6.80 & 0.20 \\
\hline 6 & 22.4 & 2.7 & 0.9 & 0.8 & 8.5 & 0 & 64.7 & 10.1 & 0.10 & 0.01 & 7.00 & 0.20 \\
\hline 7 & 260.3 & 1.3 & 96.3 & 1.6 & 37.9 & 0 & 288.0 & 575.9 & 0.45 & 0.28 & 16.25 & 0.56 \\
\hline 8 & 132.9 & 0.5 & 71.9 & 1.2 & 27.6 & 0 & 331.3 & 109.0 & 0.10 & 0.08 & 8.00 & 0.24 \\
\hline 9 & 18.6 & 0.1 & 0.5 & 0.4 & 6.7 & 0 & 45.8 & 5.8 & 0.10 & 0.01 & 5.70 & 0.20 \\
\hline 10 & 138.5 & 0.7 & 30.6 & 14.1 & 5.3 & 0 & 363.7 & 122.0 & 0.10 & 0.05 & 16.25 & 0.98 \\
\hline 11 & 115.8 & 0.5 & 22.1 & 7.0 & 5.3 & 0 & 213.6 & 164.7 & 0.10 & 0.02 & 22.75 & 1.30 \\
\hline 12 & 38.3 & 0.1 & 0.5 & 0.8 & 3.5 & 0 & 114.7 & 5.8 & 0.10 & 0.02 & 5.25 & 0.20 \\
\hline 13 & 28.8 & 0.1 & $0 . \ddot{1}$ & 0.8 & 3.5 & 0 & 103.1 & 4.8 & 0.10 & 0.01 & 4.20 & 0.20 \\
\hline 14 & 30.3 & 0.6 & 7.3 & 0.4 & 3.5 & 0 & 109.8 & 3.8 & 0.10 & 0.01 & 7.50 & 0.20 \\
\hline 15 & 42.1 & 0.4 & 1.6 & 1.2 & 3.5 & 0 & 89.1 & 50.0 & 0.10 & 0.01 & 9.05 & 0.20 \\
\hline 16 & 37.3 & 0.1 & 0.5 & 0.4 & 3.5 & 0 & 124.5 & 5.8 & 0.10 & 0.01 & 5.75 & 0.20 \\
\hline 17 & 427.1 & 2.5 & 17.7 & 3.1 & 27.6 & 0 & 245.3 & 891.9 & 0.10 & 0.17 & 14.85 & 1.75 \\
\hline 18 & 35.3 & 0.2 & 0.7 & 0.8 & 3.5 & 0 & 104.3 & 10.1 & 0.10 & 0.01 & 5.15 & 0.20 \\
\hline 19 & 16.0 & 0.1 & 1.6 & 1.2 & 3.5 & 0 & 48.2 & 10.1 & 0.10 & 0.01 & 4.35 & 0.20 \\
\hline 20 & 59.5 & 0.2 & 1.6 & 0.8 & 3.5 & 0 & 183.0 & 10.1 & 0.10 & 0.01 & 5.15 & 0.20 \\
\hline 21 & 24.8 & 0.2 & 1.4 & 0.8 & 3.5 & 0 & 73.2 & 10.1 & 0.10 & 0.01 & 4.90 & 0.20 \\
\hline 22 & 8.2 & 3.3 & 3.0 & 2.3 & 5.3 & 0 & 46.4 & 2.9 & 0.10 & 0.05 & 13.80 & 0.20 \\
\hline 23 & 3.6 & 1.2 & 2.1 & 0.8 & 3.5 & 0 & 17.1 & 4.8 & 0.10 & 0.01 & 11.00 & 0.20 \\
\hline 24 & 101.2 & 0.2 & 2.5 & 2.0 & 6.4 & 0 & 308.7 & 12.0 & 0.10 & 0.01 & 13.45 & 0.20 \\
\hline 25 & 55.7 & 5.3 & 1.6 & 0.8 & 5.3 & 0 & 194.0 & 5.3 & 0.10 & 0.01 & 7.35 & 0.20 \\
\hline 27 & 45.3 & 3.9 & 1.1 & 0.8 & 3.5 & 0 & 153.8 & 8.1 & 0.10 & 0.01 & 9.15 & 0.20 \\
\hline 28 & 29.8 & 2.8 & 0.9 & 0.8 & 3.5 & 0 & 97.6 & 8.1 & 0.10 & 0.02 & 9.20 & 0.20 \\
\hline 29 & 1.6 & 1.1 & 0.9 & 0.8 & 3.5 & 0 & 15.2 & 2.9 & 0.10 & 0.01 & 5.70 & 0.20 \\
\hline 145 & 35.5 & 9.7 & 22.5 & 2.7 & 0.7 & 0 & 229.4 & 2.4 & - & - & 10.24 & - \\
\hline 146 & 27.0 & 2.2 & 36.3 & 1.6 & 7.4 & 0 & 159.9 & 13.9 & - & - & 12.78 & - \\
\hline 147 & 2.8 & 0.5 & 132.9 & 0.8 & 5.0 & 0 & 339.2 & 16.8 & - & - & 8.20 & - \\
\hline 148 & 41.5 & 4.9 & 14.2 & 1.6 & 2.8 & 0 & 181.8 & 13.9 & - & - & 11.71 & - \\
\hline 149 & 26.6 & 4.9 & 72.9 & 2.7 & 38.3 & 0 & 233.1 & 2.4 & - & - & 10.63 & - \\
\hline 150 & 9.4 & 5.1 & 48.5 & 3.5 & 87.9 & 0 & 17.7 & 5.3 & - & - & 8.29 & - \\
\hline 151 & 3.6 & 1.4 & 0.9 & 1.6 & 5.0 & 0 & 11.0 & 5.3 & - & - & 9.07 & \\
\hline 152 & 21.0 & 5.5 & 12.9 & 3.5 & 9.9 & 0 & 115.9 & & - & - & 11.32 & - \\
\hline 153 & 30.4 & 6.2 & 30.6 & 2.7 & 7.8 & 0 & 183.0 & 9.1 & - & - & 11.32 & - \\
\hline 154 & 63.1 & 9.2 & 10.6 & 1.6 & 2.1 & 0 & 191.6 & 63.4 & - & - & 13.37 & - \\
\hline 155 & 12.6 & 1.7 & 239.8 & 1.2 & 169.5 & 9.6 & 342.9 & 13.9 & - & - & 9.27 & - \\
\hline 156 & 33.7 & 9.7 & 12.0 & 1.2 & 2.1 & 0 & 148.9 & 20.2 & - & - & 8.68 & - \\
\hline 157 & 1.6 & 0.2 & 128.0 & 0.4 & 6.7 & 19.2 & 259.9 & 11.5 & - & - & 10.54 & - \\
\hline
\end{tabular}


TABLE II (cont.)

\begin{tabular}{|c|c|c|c|c|c|c|c|c|c|c|c|c|}
\hline $\begin{array}{c}\text { Sample } \\
\text { No. }\end{array}$ & $\mathrm{Ca}$ & $M g$ & $\mathrm{Na}$ & $K$ & $\mathrm{Cl}$ & $\mathrm{CO}_{3}$ & $\mathrm{HCO}_{3}$ & $\mathrm{SO}_{4}$ & $\mathrm{Fe}$ & B & SiO & $\mathrm{F}$ \\
\hline 158 & 106.4 & 17.5 & 13.3 & 0.8 & 148.2 & 0 & 147.7 & 28.8 & - & - & 19.71 & - \\
\hline 159 & 5.2 & 1.4 & 112.0 & 1.2 & 11.0 & 28.8 & 219.7 & 16.8 & - & - & 9.17 & - \\
\hline 160 & 13.6 & 4.2 & 6.9 & 1.6 & 1.4 & 0 & 81.8 & 2.4 & - & - & 12.98 & - \\
\hline 161 & 9.4 & 5.1 & 2.1 & 2.3 & 9.9 & 0 & 40.3 & 5.3 & - & - & 8.00 & - \\
\hline 162 & 1.2 & 0.2 & 210.3 & 0.4 & 21.6 & 40.8 & 430.8 & 9.1 & - & - & 8.49 & - \\
\hline 163 & 6.0 & 1.2 & 162.1 & 1.2 & 57.4 & 0 & 361.2 & 5.3 & - & - & 9.27 & - \\
\hline 164 & 15.4 & 4.0 & 2.3 & 1.2 & 1.8 & 0 & 45.1 & 26.9 & - & - & 8.10 & - \\
\hline 165 & 11.0 & 2.3 & 69.0 & 1.2 & 17.7 & 0 & 173.3 & 9.1 & - & - & 11.61 & - \\
\hline 166 & 51.3 & 10.2 & 25.5 & 2.0 & 29.4 & 0 & 220.9 & 16.8 & - & - & 12.20 & - \\
\hline 167 & 70.9 & 23.8 & 13.3 & 2.0 & 26.2 & 0 & 324.6 & 11.5 & - & - & 16.50 & - \\
\hline 168 & 13.6 & 3.0 & 54.9 & 3.0 & 0.8 & 0 & 155.0 & 9.1 & - & - & 14.50 & - \\
\hline 169 & 1.2 & 1.4 & 167.8 & 0.8 & 72.7 & 0 & 289.2 & 23.0 & - & - & 9.07 & - \\
\hline 170 & 3.2 & 0.5 & 91.0 & 0.2 & 30.1 & 0 & 168.4 & 23.0 & - & - & 9.76 & - \\
\hline 171 & 11.0 & 1.8 & 118.2 & 1.2 & 35.8 & 0 & 279.4 & 23.0 & - & - & 13.95 & - \\
\hline 172 & 47.3 & 7.5 & 25.0 & 1.6 & 36.5 & 0 & 184.3 & 13.9 & - & - & 16.50 & - \\
\hline 173 & 2.0 & 0.2 & 154.7 & 0.4 & 14.5 & 34.8 & 286.8 & 16.8 & - & - & 9.27 & - \\
\hline 174 & 64.3 & 27.3 & 30.1 & 2.0 & 7.1 & 0 & 384.4 & 33.6 & - & - & 15.20 & - \\
\hline 175 & 68.5 & 17.5 & 7.8 & 1.2 & 7.1 & 0 & 273.3 & 25.0 & - & - & 11.00 & - \\
\hline 176 & 7.2 & 1.8 & 80.2 & 1.2 & 3.9 & 0 & 217.2 & 11.5 & - & - & 11.80 & - \\
\hline 177 & 76.0 & 9.5 & 21.1 & 1.6 & 7.1 & 0 & 272.1 & 46.6 & - & - & 15.90 & - \\
\hline 178 & 29.7 & 4.5 & 58.2 & 1.6 & 3.2 & 0 & 244.1 & 23.0 & - & - & 14.60 & - \\
\hline 179 & 15.2 & 4.1 & 20.2 & 5.1 & 2.5 & 0 & 59.8 & 48.5 & - & - & 10.25 & - \\
\hline 180 & 26.2 & 5.2 & 6.2 & 0.8 & 1.8 & 0 & 103.7 & 13.9 & - & - & 22.45 & - \\
\hline 181 & 16.0 & 6.9 & 5.7 & 1.6 & 8.5 & 0 & 64.7 & 13.9 & - & - & 9.90 & - \\
\hline 182 & 77.7 & 21.5 & 53.1 & 1.2 & 12.0 & 30.0 & 350.2 & 44.2 & - & - & 15.90 & - \\
\hline 183 & 6.4 & 1.0 & 213.3 & 0.1 & 181.5 & 0 & 238.0 & 16.8 & - & - & 9.61 & - \\
\hline 184 & 33.9 & 9.1 & 23.0 & 1.6 & 2.5 & 0 & 191.6 & 13.9 & - & - & 5.80 & - \\
\hline 185 & 32.5 & 4.6 & 32.2 & 1.6 & 6.7 & 0 & 184.3 & 16.8 & - & - & 15.80 & \\
\hline 186 & 28.0 & 4.9 & 11.0 & 2.0 & 3.2 & 0 & 89.1 & 39.9 & - & - & 8.59 & - \\
\hline 187 & 48.7 & 8.0 & 41.4 & 1.6 & 38.6 & 0 & 208.7 & 28.8 & - & - & 12.20 & - \\
\hline 188 & 26.8 & 6.0 & 45.5 & 1.6 & 31.2 & 0 & 150.1 & 23.0 & - & - & 10.30 & - \\
\hline 189 & 69.3 & 20.2 & 30.8 & 2.0 & 11.7 & 0 & 351.4 & 37.5 & - & - & 11.85 & - \\
\hline 190 & 93.2 & 32.0 & 37.2 & 2.3 & 152.4 & 0 & 235.5 & 50.4 & - & - & 10.80 & - \\
\hline
\end{tabular}


TABLE II I

CHEMICAL ANALYSES OF SPRINGS AND WELLS IN WEST VIRGINIA, meg/ $\ell$

\begin{tabular}{|c|c|c|c|c|c|c|c|c|c|c|c|c|}
\hline $\begin{array}{l}\text { Sample } \\
\text { No. }\end{array}$ & $\begin{array}{c}\text { monhos } / \mathrm{cm} \\
\mathrm{EC}\end{array}$ & $\mathrm{pH}$ & $\mathrm{Ca}$ & $\mathrm{Na}$ & K & $\mathrm{Mg}$ & $\begin{array}{l}\text { Total } \\
\text { Cation } \\
\end{array}$ & $\mathrm{Cl}$ & $\mathrm{CO}_{3}$ & $\mathrm{HCO}_{3}$ & $\mathrm{SO}_{4}$ & $\begin{array}{l}\text { Total } \\
\text { Anion }\end{array}$ \\
\hline 1 & 0.236 & 7.41 & 2.01 & 0.06 & 0.03 & 0.02 & 2.12 & 0.16 & 0 & 2.06 & 0.12 & 2.34 \\
\hline 2 & 0.083 & 7.41 & 0.47 & 0.11 & 0.03 & 0.14 & 0.75 & 0.10 & 0 & 0.58 & 0.14 & 0.72 \\
\hline 3 & 0.244 & 7.27 & 1.96 & 0.06 & 0.03 & 0.02 & 2.07 & 0.16 & 0 & 2.04 & 0.15 & 2.35 \\
\hline 4 & 1.590 & 6.66 & 15.65 & 0.86 & 0.65 & 0.10 & 17.26 & 0.58 & 0 & 5.02 & 10.99 & 16.81 \\
\hline 5 & 0.247 & 7.33 & 2.37 & 0.03 & 0.02 & 0.01 & 2.42 & 0.10 & 0 & 2.26 & 0.19 & 2.45 \\
\hline 6 & 0.144 & 7.51 & 1.12 & 0.04 & 0.02 & 0.22 & 1.40 & 0.24 & 0 & 1.06 & 0.21 & 1.51 \\
\hline 7 & 1.590 & 7.09 & 12.99 & 4.10 & 0.04 & 0.11 & 17.33 & 1.07 & 0 & 4.72 & 11.99 & 17.78 \\
\hline 8 & 0.898 & 7.28 & 6.63 & 3.13 & 0.03 & 0.04 & 9.83 & 0.78 & 0 & 5.43 & 2.27 & 9.48 \\
\hline 9 & 0.102 & 7.35 & 0.93 & 0.02 & 0.01 & 0.01 & 0.97 & 0.19 & 0 & 0.75 & 0.12 & 1.06 \\
\hline 10 & 0.846 & 7.65 & 6.91 & 1.33 & 0.36 & 0.06 & 8.66 & 0.15 & 0 & 5.96 & 2.54 & 8.65 \\
\hline 11 & 0.698 & 7.57 & 5.78 & 0.96 & 0.18 & 0.04 & 6.96 & 0.15 & 0 & 3.50 & 3.43 & 7.18 \\
\hline 12 & 0.198 & 7.69 & 1.91 & 0.02 & 0.02 & 0.01 & 1.96 & 0.10 & 0 & 1.88 & 0.12 & 2.00 \\
\hline 13 & 0.150 & 7.58 & 1.44 & 0.03 & 0.02 & 0.01 & 1.50 & 0.10 & 0 & 1.69 & 0.10 & 1.79 \\
\hline 14 & 0.194 & 7.85 & 1.51 & 0.32 & 0.01 & 0.05 & 1.89 & 0.10 & 0 & 1.80 & 0.08 & 1.88 \\
\hline 15 & 0.263 & 7.75 & 2.10 & 0.07 & 0.03 & 0.03 & 2.23 & 0.10 & 0 & 1.46 & 1.04 & 2.50 \\
\hline 16 & 0.189 & 7.22 & 1.86 & 0.02 & 0.01 & 0.01 & 1.90 & 0.10 & 0 & 2.04 & 0.12 & 2.16 \\
\hline 17 & 2.030 & 7.50 & 21.31 & 0.77 & 0.08 & 0.21 & 22.37 & 0.78 & 0 & 4.02 & 18.57 & 23.37 \\
\hline 18 & 0.203 & 7.59 & 1.76 & 0.03 & 0.02 & 0.02 & 1.83 & 0.10 & 0 & 1.71 & 0.21 & 1.92 \\
\hline 19 & 0.110 & 7.10 & 0.80 & 0.07 & 0.03 & 0.01 & 0.91 & 0.10 & 0 & 0.79 & 0.21 & 1.00 \\
\hline 20 & 0.310 & 7.84 & 2.97 & 0.07 & 0.02 & 0.02 & 3.08 & 0.10 & 0 & 3.00 & 0.21 & 3.21 \\
\hline 21 & 0.157 & 7.35 & 1.24 & 0.06 & 0.02 & 0.02 & 1.34 & 0.10 & 0 & 1.20 & 0.21 & 1.41 \\
\hline 22 & 0.101 & 6.04 & 0.41 & 0.13 & 0.06 & 0.27 & 0.87 & 0.15 & 0 & 0.76 & 0.06 & 0.97 \\
\hline 23 & 0.037 & 5.48 & 0.18 & 0.09 & 0.02 & 0.10 & 0.39 & 0.10 & 0 & 0.28 & 0.10 & 0.38 \\
\hline 24 & 0.511 & 7.30 & 5.05 & 0.11 & 0.05 & 0.02 & 5.23 & 0.18 & 0 & 5.06 & 0.25 & 5.49 \\
\hline 25 & 0.336 & 7.47 & 2.78 & 0.07 & 0.02 & 0.44 & 3.31 & 0.15 & 0 & 3.18 & 0.11 & 3.44 \\
\hline 27 & 0.259 & 7.43 & 2.26 & 0.05 & 0.02 & 0.32 & 2.55 & 0.10 & 0 & 2.52 & 0.17 & 2.69 \\
\hline 28 & 0.179 & 7.38 & 1.49 & 0.04 & 0.02 & 0.09 & 1.78 & 0.10 & 0 & 1.60 & 0.17 & 1.77 \\
\hline 29 & 0.028 & 5.60 & 0.08 & 0.04 & 0.02 & 0.09 & 0.23 & 0.10 & 0 & 0.25 & 0.06 & 0.25 \\
\hline 145 & 0.314 & 8.12 & 1.77 & 0.98 & 0.07 & 0.80 & 3.62 & 0.02 & 0 & 3.76 & 0.05 & 3.83 \\
\hline 146 & 0.285 & 8.00 & 1.35 & 1.58 & 0.04 & 0.18 & 3.15 & 0.21 & 0 & 2.62 & 0.29 & 3.18 \\
\hline 147 & 0.533 & 8.33 & 0.14 & 5.78 & 0.02 & 0.04 & 5.98 & 0.14 & 0 & 5.56 & 0.35 & 6.05 \\
\hline 148 & 0.284 & 8.18 & 2.07 & 0.62 & 0.04 & 0.40 & 3.13 & 0.08 & 0 & 2.98 & 0.29 & 3.35 \\
\hline 149 & 0.446 & 7.96 & 1.33 & 3.17 & 0.07 & 0.40 & 4.97 & 1.08 & 0 & 3.82 & 0.05 & 4.95 \\
\hline 150 & 0.344 & 6.73 & 0.47 & 2.11 & 0.09 & 0.42 & 3.09 & 2.48 & 0 & 0.29 & 0.11 & 2.88 \\
\hline 151 & 0.045 & 6.72 & 0.18 & 0.04 & 0.04 & 0.12 & 0.38 & 0.14 & 0 & 0.18 & 0.11 & 0.43 \\
\hline 152 & 0.207 & 8.12 & 1.05 & 0.56 & 0.09 & 0.45 & 2.15 & 0.28 & 0 & 1.90 & 0.11 & 2.29 \\
\hline 153 & 0.307 & 8.32 & 1.54 & 1.33 & 0.07 & 0.51 & 3.45 & 0.22 & 0 & 3.00 & 0.19 & 3.41 \\
\hline 154 & 0.404 & 8.34 & 3.15 & 0.46 & 0.04 & 0.76 & 4.41 & 0.06 & 0 & 3.14 & 1.32 & 4.52 \\
\hline 155 & 1.030 & 8.46 & 0.63 & 10.43 & 0.03 & 0.14 & 11.23 & 4.78 & 0.32 & 5.62 & 0.29 & 11.01 \\
\hline 156 & 0.274 & 8.33 & 1.68 & 0.52 & 0.03 & 0.80 & 3.03 & 0.06 & 0 & 2.44 & 0.42 & 2.93 \\
\hline 157 & 0.461 & 8.50 & 0.08 & 5.57 & 0.01 & 0.02 & 5.68 & 0.19 & 0.64 & 4.26 & 0.24 & 5.33 \\
\hline
\end{tabular}


TABLE II (cont.)

\begin{tabular}{|c|c|c|c|c|c|c|c|c|c|c|c|c|}
\hline $\begin{array}{l}\text { Sample } \\
\text { No. }\end{array}$ & $\begin{array}{c}\text { manhos } / \mathrm{cm} \\
E C\end{array}$ & $\mathrm{pH}$ & $\mathrm{Ca}$ & $\mathrm{Na}$ & $k$ & $\mathrm{Mg}$ & $\begin{array}{l}\text { Total } \\
\text { Cation }\end{array}$ & $\mathrm{Cl}$ & $\mathrm{CO}_{3}$ & $\mathrm{HCO}_{3}$ & $\mathrm{SO}_{4}$ & $\begin{array}{l}\text { Total } \\
\text { Anion }\end{array}$ \\
\hline 158 & 0.788 & 7.92 & 5.31 & 0.58 & 0.02 & 1.44 & 7.35 & 4.18 & 0 & 2.42 & 0.60 & 7.20 \\
\hline 159 & 0.469 & 8.87 & 0.26 & 4.87 & 0.03 & 0.12 & 5.28 & 0.31 & 0.96 & 3.60 & 0.35 & 5.22 \\
\hline 160 & 0.135 & 7.66 & 0.68 & 0.30 & 0.04 & 0.35 & 1.37 & 0.04 & 0 & 1.34 & 0.05 & 1.43 \\
\hline 161 & 0.124 & 7.09 & 0.47 & 0.09 & 0.06 & 0.42 & 1.04 & 0.28 & 0 & 0.66 & 0.11 & 1.05 \\
\hline 162 & 0.772 & 8.93 & 0.06 & 9.15 & 0.01 & 0.02 & 9.24 & 0.61 & 1.36 & 7.06 & 0.19 & 9.22 \\
\hline 163 & 0.695 & 8.27 & 0.30 & 7.05 & 0.03 & 0.10 & 7.48 & 1.62 & 0 & 5.92 & 0.11 & 7.68 \\
\hline 164 & 0.132 & 7.59 & 0.77 & 0.10 & 0.03 & 0.33 & 1.23 & 0.05 & 0 & 0.74 & 0.56 & 1.35 \\
\hline 165 & 0.327 & 8.12 & 0.55 & 3.00 & 0.03 & 0.19 & 3.77 & 0.50 & 0 & 2.84 & 0.19 & 3.53 \\
\hline 166 & 0.440 & 8.25 & 2.56 & 1.11 & 0.05 & 0.84 & 4.56 & 0.83 & 0 & 3.62 & 0.35 & 4.80 \\
\hline 167 & 0.573 & 7.93 & 3.54 & 0.58 & 0.05 & 1.96 & 6.13 & 0.74 & 0 & 5.32 & 0.24 & 6.30 \\
\hline 168 & 0.289 & 8.30 & 0.68 & 2.39 & 0.02 & 0.25 & 3.34 & 0.46 & 0 & 2.54 & 0.19 & 3.19 \\
\hline 169 & 0.656 & 8.27 & 0.06 & 7.30 & 0.02 & 0.12 & 7.50 & 2.05 & 0 & 4.74 & 0.48 & 7.27 \\
\hline 170 & 0.378 & 8.24 & 0.16 & 3.96 & 0.01 & 0.04 & 4.17 & 0.85 & 0 & 2.76 & 0.48 & 4.09 \\
\hline 171 & 0.520 & 8.23 & 0.55 & 5.14 & 0.03 & 0.15 & 5.87 & 1.01 & 0 & 4.58 & 0.48 & 6.07 \\
\hline 172 & 0.407 & 8.08 & 2.36 & 1.09 & 0.04 & 0.62 & 4.11 & 1.03 & 0 & 3.02 & 0.29 & 4.34 \\
\hline 173 & 0.569 & 8.78 & 0.10 & 6.73 & 0.01 & 0.02 & 6.86 & 0.41 & 1.16 & 4.70 & 0.35 & 6.62 \\
\hline 174 & 0.578 & 8.13 & 3.21 & 1.31 & 0.05 & 2.25 & 6.82 & 0.20 & 0 & 6.30 & 0.70 & 7.20 \\
\hline 175 & 0.489 & 8.36 & 3.42 & 0.34 & 0.03 & 1.44 & 5.23 & 0.20 & 0 & 4.48 & 0.52 & 5.20 \\
\hline 176 & 0.342 & 8.33 & 0.36 & 3.49 & 0.03 & 0.15 & 4.03 & 0.11 & 0 & 3.56 & 0.24 & 3.91 \\
\hline 177 & 0.498 & 8.08 & 3.79 & 0.92 & 0.04 & 0.78 & 5.53 & 0.20 & 0 & 4.4 & 0.97 & 5.63 \\
\hline 178 & 0.370 & 8.30 & 1.48 & 2.53 & 0.04 & 0.37 & 4.59 & 0.09 & 0 & 4.00 & 0.48 & 4.57 \\
\hline 179 & 0.221 & 7.55 & 0.76 & 0.88 & 0.13 & 0.34 & 2.11 & 0.07 & 0 & 0.98 & 1.01 & 2.06 \\
\hline 180 & 0.190 & 8.24 & 1.31 & 0.27 & 0.02 & 0.43 & 2.03 & 0.05 & 0 & 1.70 & 0.29 & 2.04 \\
\hline 181 & 0.180 & 7.50 & 0.80 & 0.25 & 0.04 & 0.57 & 1.66 & 0.24 & 0 & 1.06 & 0.29 & 1.54 \\
\hline 182 & 0.647 & 8.53 & 3.88 & 2.31 & 0.03 & 1.77 & 7.99 & 0.34 & 1.00 & 5.74 & 0.92 & 8.00 \\
\hline 183 & 0.921 & 8.29 & 0.32 & 9.28 & 0.03 & 0.08 & 9.71 & 5.21 & 0 & 3.90 & 0.35 & 9.37 \\
\hline 184 & 0.310 & 8.26 & 1.69 & 1.00 & 0.04 & 0.75 & 3.48 & 0.07 & 0 & 3.14 & 0.29 & 3.50 \\
\hline 185 & 0.310 & 8.32 & 1.62 & 1.40 & 0.04 & 0.38 & 3.44 & 0.19 & 0 & 3.02 & 0.35 & 3.56 \\
\hline 186 & 0.231 & 7.92 & 1.40 & 0.48 & 0.05 & 0.40 & 2.33 & 0.09 & 0 & 1.46 & 0.83 & 2.30 \\
\hline 187 & 0.472 & 7.93 & 2.43 & 1.80 & 0.04 & 0.66 & 4.93 & 1.09 & 0 & 3.42 & 0.60 & 5.11 \\
\hline 188 & 0.359 & 8.12 & 1.34 & 1.98 & 0.04 & 0.49 & 3.85 & 0.88 & 0 & 2.46 & 0.48 & 3.82 \\
\hline 189 & 0.573 & 8.07 & 3.46 & 1.34 & 0.05 & 1.66 & 6.51 & 0.33 & 0 & 5.76 & 0.78 & 6.87 \\
\hline 190 & 0.882 & 8.18 & 4.65 & 1.62 & 0.06 & 2.63 & 8.96 & 4.30 & 0 & 3.86 & 1.05 & 9.21 \\
\hline
\end{tabular}


In addition, Sass et al. (1976) show a zone of low heat flow (<1.0 HFU) in extreme eastern West Virginia and adjacent portions of Virginia. Both the silica heat flow and the traditional heat flow (Perry and Costain 1979) have higher values to the west of the Valley and Ridge province in the Allegheny Plateau. The excellent correlation between silica heat flow and traditional heat flow for the Allegheny Plateau in northern West Virginia is noteworthy.

Silica heat-flow values were averaged over the regions of hightemperature gradients as determined by analyses of bottom-hole temperatures from oil and gas wells (AAPG 1976). Areas of high silica heat flow and areas of high bottom-hole temperature gradients do not correlate in West Virginia.

The temperature gradient map of Guffanti and Natheson (1980), updated from the AAPG (1976) map, shows a more extensive area of elevated temperature gradients including an area in western West Virginia which has high silica heat flow. However, the high-temperature gradients in central West Virginia are not shown by the silica geothermometric data.

The silica heat flow has been studied within the main ground-water basins of West Virginia for which abundant geochemical data are available. The same persistent trend is observed in this grouping whereby the lowest heat-flow values are in the east and the higher ones are in the west.

Site Specific Anomalies. Geothermal evaluation of the geochemical data can be used in the search for specific geothermal anomalies as described by Swanberg and Alexander (1979). Histograms of the silica and NaKCa geothermometers were prepared and the mean and standard deviation for each geothermometer were calculated. Values in excess of one standard deviation above the mean were assumed to be of potential geothermal origin. These potential geothermal waters were plotted against the remainder of the data to determine trends. If the anomalously high values for both geothermometers tended to cluster in a particular area, a true geothermal prospect was indicated and further geophysical studies were warranted. Also, the anomalously high values may have reflected geologic structures such as faults, ground-water discharge points, basin constrictions, or other features which control the flow of geothermal fluids. In the present data set, the anomalously high values tend to be dispersed with the more normal values. Further, the two geothermometers do not correlate well. Thus the geochemical data do not indicate any major geothermal anomalies in West Virginia. 


\section{BOUGUER ANOMALIES OF WEST VIRGINIA}

The Bouguer anomalies throughout West Virginia, tabulated in Appendix $B$ and shown in Fig. 2, are caused by crustal thickness variations, basement relief, and lateral density contrasts in the Paleozoic strata (Kulander and Dean 1978). Along a west-east profile, Bouguer anomaly values are generally about $-50 \mathrm{mgals}$ at the Ohio border, rise over the west-central area, and decrease to $-80 \mathrm{mgals}$ near the Virginia border. The central gravity ridge which is shown as the pattern in Fig. 2 reaches values as high as -46 mgals and seems asymmetric. The crest of this positive anomaly centers over the Rome Trough, trends $\mathrm{N} 30-35^{\circ} \mathrm{E}$, and parallels structural axes. This positive gravity feature is positioned over the deepest sedimentary basin in West Virginia. Other positive Bouguer anomalies throughout the state trend northeast to north.

A two-dimensional gravity model indicates a high-density body occurs near the basement interface on the eastern flank of the Rome Trough. The model also indicates that a positive density contrast near the crust-mantle interface may explain the broad gravity high in central West Virginia. Kulander and Dean (1978) suggest that the eastern flank of the Rome Trough is bounded by a major displacement in the basement, where the basement is downthrown approximately $6000 \mathrm{ft}(1800 \mathrm{~m})$. This region shows a correlation of gravity, magnetic, and temperature gradients. The Rome Trough may be the site of an incipient rift in Early Cambrian (Avalonian) time that subsequently was filled with sedimentary rocks to form the thick sedimentary basins.

\section{ACKNOWLEDGMENTS}

A. C. Waters critically reviewed the manuscript. 


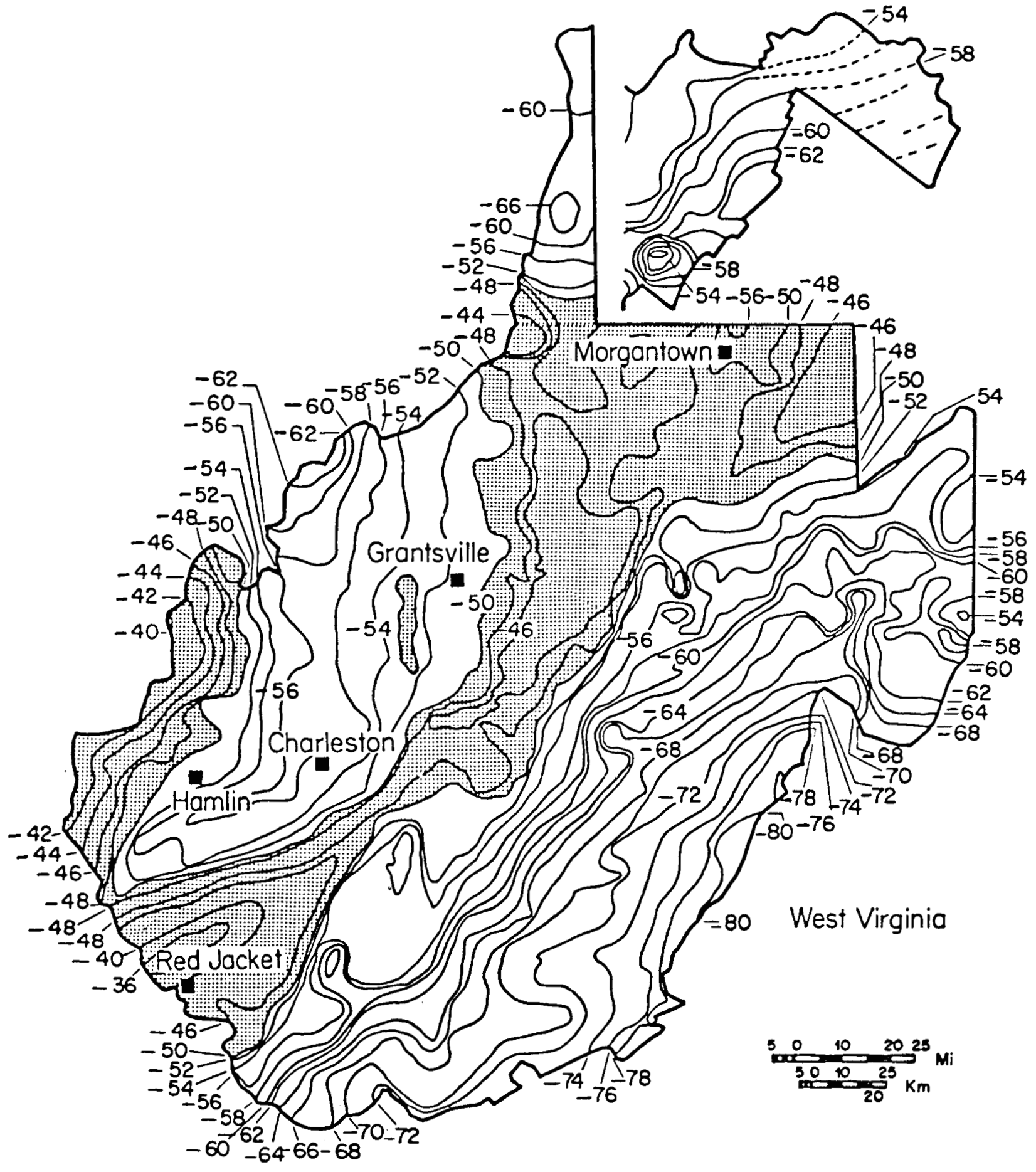

Fig. 2.

Bouguer anomaly map (2 mgal contour) of West Virginia. The shaded area encloses values greater than $-50 \mathrm{mgal}$. 


\section{REFERENCES}

AAPG-USGS Subsurface temperature of North America, 1976, U.S. Geol. Survey.

Ammerman, M. L. and Keller, G. R., 1979, Delineation of Rome Trough in Eastern Kentucky by gravity and deep drilling data, AAPG, v. 63, 341-353.

Bass, M. N., 1959, Basement rocks from the Sandhill well, Wood County, West Virginia, West Virginia Geol. Surv. Rpt. Invest. 18, p. 145-168.

Fournier, R. 0. and Rowe, J. J., 1966, Estimation of underground temperature from the silica content of water from hot springs and wet steam wells, Am. Jour. Sci., v. 264, no. 9, 685-697.

Fournier, R. 0. and Truesdel1, A. N., 1973, An empirical Na-K-Ca geothermometer for natural waters, Geochim. et Cosmoch. Acta, v. 37, no. 5, p. $1255-1275$.

Guffanti, M. and Nathenson, M., 1980, Preliminary map of temperature gradients in the conterminous United States, Geotherm. Resour. Coun. Trans., v. 4, 53-56.

Hilifiker, K., Hanley, C., Hodge, D., Morgan, P., Maxwell, J., and Swanberg, C. A., 1980, Geothermal Resources Council, Transactions, v. 4, p. 209-212.

Hobba, W. A. Jr., Chemerys, J. C., Fisher, D. W., and Pearson, F. J. Jr., 1977, Geochemical and hydrological data for wells and springs in thermal spring areas of the Appalachians, U.S. Geol. Survey, Water-Resources Invest. $77-25$, p. 1-36.

Hodge, D. S., Hilfiker, K., Morgan, P., and Swanberg, C. A., 1979, Preliminary geothermal investigations in New York State, Geotherm. Resour. Coun. Trans., v. 3, p. 317-320.

King, E. R. and I. Zietz, 1978, The New York-Alabama lineament: Geophysical evidence for a major crustal break in the basement beneath the Appalachian Basin, Geology, v. 6, p. 312-318.

Kulander, B. R. and S. L. Dean, 1978, Gravity magnetics, and structure, Allegheny Plateau/Western Valley and Ridge in West Virginia and adjacent states: Rpt. Invest. 27, West Virginia Geological and Economic Survey, 91 p.

Perry, L. D. and Costain, J. K., 1979, Heat flow in western Virginia and a model for the origin of thermal springs in the folded Appalachians, $J$. Geophys. Res., v. 84, p. 6875-6883.

Sass, J. H., Diment, W. H., Lachenbruch, A. H., Marshall, B. V., Munroe, R. J., Moses, T. H. Jr., and Urban, T. C., 1976, A new heat flow contour map of the conterminous United States, U.S. Geol. Survey, Open-File Rpt. $76-756,24 \mathrm{p}$. 
Swanberg, C. A. and Alexander, S., 1979, The use of the water quality file WATSTORE in geothermal exploration: An example from the Imperial Valley, California, Geology, v. 7, 108-11l.

Swanberg, C. A. and Morgan, P., 1978/1979, The linear relation between temperatures based on the silica content of groundwater and regional heat flow. A new heat flow map of the U.S., Pure and Applied Geophysics, v. $117(1 / 2), 227-241$.

Swanberg, C. A. and Morgan, P., 1980, The silica heat flow interpretation technique: Assumptions and applications, J. Geophys. Res., v. 85, p. $7206-7214$.

Waring, G. A., 1965, Thermal springs of the United States and other countries of the world--a summary, U.S. Geol. Survey Prof. Paper 492, 383 p. 
APPENDIX A

BOTTOM-HOLE TEMPERATURE DATA FOR WELLS IN WEST VIRGINIA

D. S. Hodge

State University of New York at Buffalo Geological Sciences

\section{Barbour County}

\begin{tabular}{|c|c|c|c|c|c|c|c|c|c|c|c|c|c|}
\hline $\begin{array}{c}\text { WELLL } \\
\text { NO }\end{array}$ & & $\begin{array}{l}\mathrm{DA} \\
1,0\end{array}$ & & $\begin{array}{l}\text { L } \Lambda \text { TITUDE } \\
\text { DFGREES }\end{array}$ & $\begin{array}{l}\text { LONGITUDE } \\
\text { DEGREFS }\end{array}$ & $\begin{array}{c}\text { ELEV } \\
M\end{array}$ & $\begin{array}{c}\text { KBELV } \\
M\end{array}$ & $\begin{array}{c}\text { DEPTH } \\
\mathbf{M}\end{array}$ & $\begin{array}{c}\mathrm{BHT} \\
\mathrm{C}\end{array}$ & $\begin{array}{c}\text { STEMP } \\
\mathrm{C}\end{array}$ & $\begin{array}{c}\text { GRADI ENT } \\
\text { C/KM }\end{array}$ & $\begin{array}{c}\text { BHTCOR } \\
\text { C }\end{array}$ & $\begin{array}{l}\text { AAPGRAD } \\
\text { C/RM }\end{array}$ \\
\hline $\begin{array}{l}646 \\
647 \\
648 \\
650 \\
651 \\
655 \\
657 \\
658 \\
659 \\
660 \\
673 \\
681 \\
801 \\
802 \\
804 \\
814 \\
822 \\
826 \\
862 \\
865 \\
866 \\
900 \\
956 \\
958 \\
967\end{array}$ & $\begin{array}{r}5 \\
6 \\
6 \\
7 \\
7 \\
12 \\
11 \\
11 \\
12 \\
11 \\
5 \\
8 \\
3 \\
10 \\
11 \\
12 \\
12 \\
2 \\
6 \\
12 \\
7 \\
11 \\
6 \\
4 \\
7\end{array}$ & $\begin{array}{r}27 \\
16 \\
7 \\
16 \\
7 \\
2 \\
20 \\
10 \\
11 \\
20 \\
2 \\
6 \\
15 \\
28 \\
17 \\
13 \\
30 \\
6 \\
30 \\
1 \\
17 \\
20 \\
27 \\
1 \\
16\end{array}$ & $\begin{array}{l}73 \\
73 \\
73 \\
73 \\
73 \\
73 \\
73 \\
73 \\
73 \\
73 \\
74 \\
74 \\
77 \\
76 \\
76 \\
76 \\
76 \\
77 \\
77 \\
77 \\
77 \\
77 \\
78 \\
78 \\
78\end{array}$ & $\begin{array}{l}39.1048 \\
39.2434 \\
39.2440 \\
39.0691 \\
39.1442 \\
39.1241 \\
39.1911 \\
39.1835 \\
39.1035 \\
39.1200 \\
39.0751 \\
39.1368 \\
39.2361 \\
39.1123 \\
39.1881 \\
39.0262 \\
39.1417 \\
39.2322 \\
39.2096 \\
39.1059 \\
39.0987 \\
39.2114 \\
39.2066 \\
39.2292 \\
39.2231\end{array}$ & $\begin{array}{l}80.1044 \\
79.9939 \\
80.0067 \\
80.0576 \\
80.0248 \\
80.1023 \\
80.0886 \\
80.0894 \\
80.0690 \\
80.1062 \\
80.1459 \\
80.0587 \\
80.0163 \\
80.0564 \\
80.0831 \\
79.9799 \\
80.1370 \\
80.0443 \\
80.0135 \\
80.0140 \\
80.0722 \\
79.9870 \\
80.0445 \\
80.0897 \\
80.0736\end{array}$ & $\begin{array}{l}511.5 \\
465.7 \\
456.3 \\
549.6 \\
550.5 \\
473.4 \\
454.8 \\
438.9 \\
452.6 \\
430.4 \\
464.8 \\
406.6 \\
449.6 \\
506.0 \\
503.2 \\
656.2 \\
437.7 \\
385.3 \\
498.3 \\
480.1 \\
460.9 \\
393.8 \\
427.6 \\
394.7 \\
448.1\end{array}$ & $\begin{array}{l}514.8 \\
469.1 \\
460.2 \\
553.8 \\
553.8 \\
476.4 \\
458.1 \\
442.3 \\
455.7 \\
433.1 \\
469.4 \\
409.7 \\
453.2 \\
509.6 \\
506.3 \\
659.0 \\
440.1 \\
387.7 \\
500.8 \\
483.1 \\
463.3 \\
396.8 \\
432.2 \\
397.8 \\
451.1\end{array}$ & $\begin{array}{r}1325.6 \\
1271.6 \\
1318.6 \\
1201.2 \\
1288.7 \\
1362.2 \\
1403.9 \\
1385.3 \\
1177.1 \\
1300.3 \\
1316.4 \\
1183.8 \\
1317.7 \\
1274.4 \\
1432.0 \\
1283.5 \\
1387.8 \\
1277.7 \\
1278.9 \\
1479.2 \\
1176.2 \\
1162.8 \\
1327.1 \\
616.6 \\
1381.0\end{array}$ & $\begin{array}{l}39.4 \\
37.2 \\
47.8 \\
41.7 \\
38.9 \\
40.6 \\
38.3 \\
38.3 \\
36.7 \\
37.8 \\
40.6 \\
36.1 \\
40.0 \\
36.1 \\
35.0 \\
38.9 \\
40.0 \\
39.4 \\
38.9 \\
43.3 \\
37.8 \\
38.9 \\
37.2 \\
29.4 \\
41.1\end{array}$ & $\begin{array}{r}9.7 \\
10.0 \\
10.1 \\
9.4 \\
9.3 \\
10.1 \\
10.2 \\
10.3 \\
10.3 \\
10.5 \\
10.2 \\
10.7 \\
10.2 \\
9.7 \\
9.7 \\
8.4 \\
10.4 \\
10.8 \\
9.7 \\
10.0 \\
10.2 \\
10.7 \\
10.4 \\
10.7 \\
10.2\end{array}$ & $\begin{array}{l}22.4 \\
21.4 \\
28.6 \\
26.9 \\
23.0 \\
22.4 \\
20.1 \\
20.2 \\
22.4 \\
21.0 \\
23.1 \\
21.5 \\
22.6 \\
20.7 \\
17.7 \\
23.8 \\
21.3 \\
22.4 \\
22.8 \\
22.5 \\
23.4 \\
24.2 \\
20.2 \\
30.4 \\
22.4\end{array}$ & $\begin{array}{r}9.6 \\
9.3 \\
9.6 \\
8.8 \\
9.4 \\
9.9 \\
10.2 \\
10.0 \\
8.6 \\
9.5 \\
9.6 \\
8.6 \\
9.6 \\
9.3 \\
10.4 \\
9.3 \\
10.1 \\
9.3 \\
9.3 \\
10.7 \\
8.6 \\
8.5 \\
9.6 \\
4.4 \\
10.0\end{array}$ & $\begin{array}{l}29.7 \\
28.7 \\
35.8 \\
34.2 \\
30.2 \\
29.6 \\
27.3 \\
27.5 \\
29.7 \\
28.3 \\
30.3 \\
28.8 \\
29.9 \\
28.0 \\
24.9 \\
31.1 \\
28.6 \\
29.7 \\
30.1 \\
29.7 \\
30.7 \\
31.5 \\
27.5 \\
37.4 \\
29.6\end{array}$ \\
\hline
\end{tabular}

Boone County

\begin{tabular}{|c|c|c|c|c|c|c|c|c|c|c|c|}
\hline $\begin{array}{l}913 \\
915 \\
920 \\
928 \\
929 \\
932 \\
933 \\
936 \\
950 \\
955 \\
956 \\
977 \\
986 \\
992 \\
996\end{array}$ & $\begin{array}{r}8 \\
5 \\
8 \\
8 \\
1 \\
4 \\
7 \\
1 \\
2 \\
4 \\
5 \\
4 \\
11 \\
3 \\
6\end{array}$ & $\begin{array}{r}31 \\
13 \\
28 \\
29 \\
25 \\
10 \\
28 \\
16 \\
16 \\
1 \\
21 \\
19 \\
16 \\
20 \\
6\end{array}$ & $\begin{array}{l}60 \\
69 \\
61 \\
61 \\
62 \\
62 \\
69 \\
63 \\
63 \\
63 \\
63 \\
65 \\
65 \\
66 \\
66\end{array}$ & $\begin{array}{l}37.9863 \\
37.9819 \\
38.0000 \\
38.0677 \\
37.9491 \\
37.9703 \\
37.9861 \\
38.2047 \\
37.9863 \\
37.9437 \\
37.9813 \\
37.9945 \\
38.0581 \\
38.0498 \\
38.0575\end{array}$ & $\begin{array}{l}81.6754 \\
81.5888 \\
81.6667 \\
81.6036 \\
81.5783 \\
81.6060 \\
81.6460 \\
81.6644 \\
81.6182 \\
81.5820 \\
81.5368 \\
81.6874 \\
81.5171 \\
81.5321 \\
81.4965\end{array}$ & $\begin{array}{l}327.4 \\
489.8 \\
356.3 \\
273.1 \\
495.3 \\
503.2 \\
494.1 \\
253.0 \\
500.2 \\
593.8 \\
314.9 \\
338.3 \\
309.4 \\
274.3 \\
375\end{array}$ & $\begin{array}{l}327.4 \\
489.8 \\
356.3 \\
273.1 \\
495.9 \\
503.8 \\
497.1 \\
254.8 \\
501.4 \\
595.3 \\
317.3 \\
338.9 \\
311.8 \\
278.0\end{array}$ & $\begin{array}{r}903.7 \\
999.7 \\
935.4 \\
654.1 \\
1018.0 \\
736.7 \\
1026.0 \\
1545.3 \\
1030.2 \\
1103.7 \\
839.7 \\
922.0 \\
815.0 \\
792.8 \\
949.8\end{array}$ & $\begin{array}{l}34.4 \\
37.8 \\
29.4 \\
28.9 \\
27.8 \\
24.4\end{array}$ & $\begin{array}{l}12 . \\
11 . \\
12 . \\
13 .\end{array}$ & $\begin{array}{l}28.7 \\
24.9 \\
31.9\end{array}$ \\
\hline
\end{tabular}


Cabe11 County

\begin{tabular}{|c|c|c|c|c|c|c|c|c|c|c|c|}
\hline $\begin{array}{l}\text { Whit, } \\
\text { No }\end{array}$ & $\begin{array}{l}\text { DATF } \\
\text { LOG }\end{array}$ & $\begin{array}{l}\text { LATITUDE } \\
\text { DEGREES }\end{array}$ & $\begin{array}{l}\text { LONG I TUDE } \\
\text { DEGREES }\end{array}$ & $\begin{array}{c}\text { ELEV } \\
M\end{array}$ & $\underset{M}{\text { KBELV }}$ & $\begin{array}{c}\text { DEPTH } \\
M\end{array}$ & $\begin{array}{c}\text { BHT } \\
\text { C }\end{array}$ & $\begin{array}{c}\text { STEMP } \\
\mathrm{C}\end{array}$ & $\begin{array}{l}\text { GRADIENT } \\
\text { C/KM }\end{array}$ & $\begin{array}{c}\text { BHTCOR } \\
\text { C }\end{array}$ & $\begin{array}{l}\text { AAPGRAD } \\
\mathrm{C} / \mathrm{KM}\end{array}$ \\
\hline $\begin{array}{rr}532 & 11 \\
543 & 1\end{array}$ & $\begin{array}{rr}24 & 68 \\
5 & 69\end{array}$ & $\begin{array}{l}38.4337 \\
38.2878\end{array}$ & $\begin{array}{l}82.3236 \\
82.2443\end{array}$ & & $\begin{array}{l}221.9 \\
270.4\end{array}$ & 1480.1 & 38.9 & 13.0 & $\begin{array}{l}23.7 \\
17.5\end{array}$ & $\begin{array}{l}10.3 \\
10.7\end{array}$ & 24.7 \\
\hline
\end{tabular}

Calhoun County

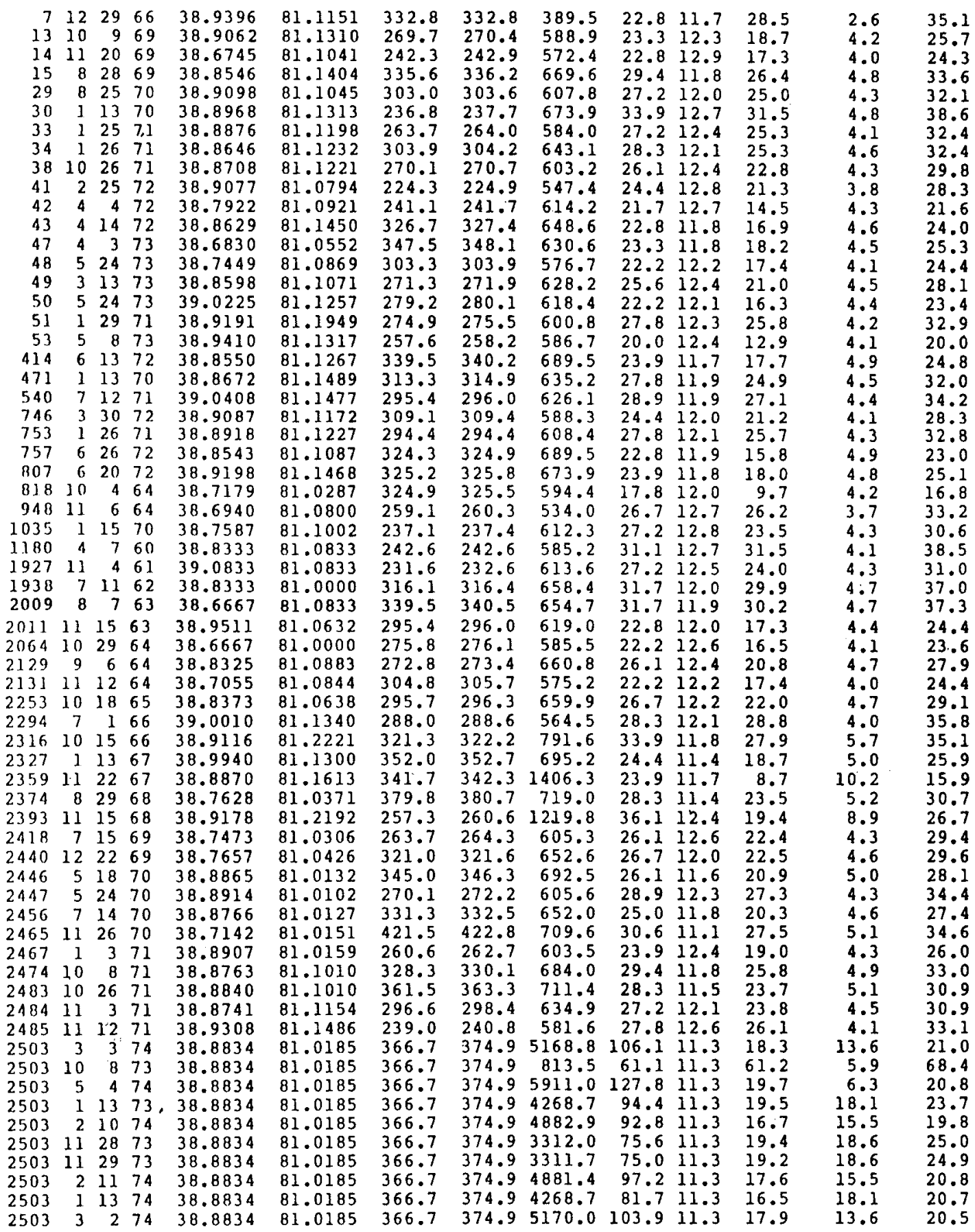


Calhoun County (cont)

\begin{tabular}{|c|c|c|c|c|c|c|c|c|c|c|c|c|c|}
\hline $\begin{array}{c}\text { WFLI. } \\
\text { NO }\end{array}$ & & $\begin{array}{l}\text { DAT } \\
\text { LOC }\end{array}$ & & $\begin{array}{l}\text { LAT ITUDE } \\
\text { DEGREES }\end{array}$ & $\begin{array}{l}\text { LONG I TUDE } \\
\text { DEGREES }\end{array}$ & $\begin{array}{c}\text { ELEV } \\
\text { M }\end{array}$ & $\underset{M}{\text { KBELV }}$ & $\begin{array}{c}\text { DEPTH } \\
M\end{array}$ & $\begin{array}{c}\text { BHT } \\
\text { C }\end{array}$ & $\begin{array}{c}\text { STEMP } \\
\mathrm{C}\end{array}$ & $\begin{array}{c}\text { GRADI ENT } \\
\text { C/RM }\end{array}$ & $\begin{array}{c}\text { BHTCOR } \\
\text { C }\end{array}$ & $\begin{array}{c}\text { AAPGRAR } \\
\text { C/RM }\end{array}$ \\
\hline $\begin{array}{l}2505 \\
2524 \\
2537 \\
2544 \\
2548 \\
2560 \\
2562 \\
2577 \\
2625 \\
2636\end{array}$ & $\begin{array}{r}6 \\
8 \\
8 \\
12 \\
12 \\
9 \\
9 \\
6 \\
4 \\
7\end{array}$ & $\begin{array}{r}21 \\
13 \\
3 \\
7 \\
14 \\
8 \\
16 \\
21 \\
13 \\
23\end{array}$ & $\begin{array}{l}73 \\
74 \\
75 \\
75 \\
75 \\
76 \\
75 \\
78 \\
78 \\
78\end{array}$ & $\begin{array}{l}38.9275 \\
38.7598 \\
38.7308 \\
38.7335 \\
38.7291 \\
38.7177 \\
38.7348 \\
38.7197 \\
38.7397 \\
38.6846\end{array}$ & $\begin{array}{l}81.2365 \\
81.0831 \\
81.1449 \\
81.0851 \\
81.1433 \\
81.1523 \\
81.1416 \\
81.1072 \\
81.1569 \\
81.0648\end{array}$ & $\begin{array}{l}306.3 \\
368.5 \\
287.1 \\
330.1 \\
281.3 \\
302.7 \\
286.5 \\
283.2 \\
262.1 \\
323.7\end{array}$ & $\begin{array}{l}308.5 \\
371.9 \\
290.5 \\
331.6 \\
284.7 \\
306.0 \\
289.9 \\
285.0 \\
265.2 \\
325.2\end{array}$ & $\begin{array}{r}651.1 \\
1904.1 \\
777.8 \\
684.6 \\
766.6 \\
676.0 \\
674.2 \\
614.5 \\
918.1 \\
602.9\end{array}$ & $\begin{array}{l}28.3 \\
51.1 \\
24.4 \\
24.4 \\
22.2 \\
23.9 \\
25.6 \\
26.1 \\
29.4 \\
26.7\end{array}$ & $\begin{array}{l}11.9 \\
11.5 \\
12.3 \\
11.9 \\
12.4 \\
12.2 \\
12.3 \\
12.4 \\
12.6 \\
12.1\end{array}$ & $\begin{array}{l}25.2 \\
20.8 \\
15.6 \\
18.3 \\
12.8 \\
17.3 \\
19.6 \\
22.3 \\
18.4 \\
24.2\end{array}$ & $\begin{array}{r}4.6 \\
13.3 \\
5.6 \\
4.9 \\
5.5 \\
4.8 \\
4.8 \\
4.4 \\
6.7 \\
4.3\end{array}$ & $\begin{array}{l}32.3 \\
27.8 \\
22.8 \\
25.4 \\
20.0 \\
24.4 \\
26.7 \\
29.4 \\
25.7 \\
31.3\end{array}$ \\
\hline
\end{tabular}

\section{Clay County}

\begin{tabular}{|c|c|c|c|c|c|c|c|c|c|c|c|c|c|}
\hline $\begin{array}{l}607 \\
622 \\
629 \\
630 \\
712 \\
810 \\
1320 \\
976 \\
1059 \\
1093 \\
1107 \\
1109 \\
1110 \\
1126 \\
1130\end{array}$ & $\begin{array}{l}4 \\
6 \\
6 \\
9 \\
3 \\
1 \\
8 \\
3 \\
5 \\
8 \\
10 \\
11 \\
10 \\
11 \\
12\end{array}$ & $\begin{array}{r}4 \\
6 \\
29 \\
5 \\
11 \\
3 \\
6 \\
30 \\
14 \\
30 \\
11 \\
1 \\
22 \\
12 \\
3\end{array}$ & $\begin{array}{l}61 \\
61 \\
61 \\
61 \\
63 \\
66 \\
66 \\
73 \\
75 \\
75 \\
75 \\
75 \\
75 \\
75 \\
75\end{array}$ & $\begin{array}{l}38.5000 \\
38.5000 \\
38.5833 \\
38.6667 \\
38.5000 \\
38.4772 \\
38.4940 \\
38.2791 \\
38.5166 \\
38.3556 \\
38.5349 \\
38.5319 \\
38.5312 \\
38.5185 \\
38.5269\end{array}$ & $\begin{array}{l}81.0833 \\
81.0833 \\
81.0000 \\
81.0000 \\
80.9167 \\
81.1842 \\
81.1479 \\
81.2153 \\
81.1630 \\
81.1518 \\
81.1504 \\
81.1583 \\
81.1648 \\
81.1632 \\
81.1532\end{array}$ & $\begin{array}{l}372.2 \\
317.0 \\
396.5 \\
258.2 \\
312.1 \\
317.6 \\
324.3 \\
406.9 \\
345.0 \\
317.0 \\
375.8 \\
308.5 \\
317.9 \\
283.8 \\
299.6\end{array}$ & $\begin{array}{l}372.8 \\
317.6 \\
397.5 \\
258.8 \\
313.0 \\
318.2 \\
324.6 \\
409.0 \\
347.5 \\
319.1 \\
377.6 \\
310.3 \\
319.7 \\
285.9 \\
301.8\end{array}$ & $\begin{array}{l}631.9 \\
623.3 \\
780.9 \\
667.8 \\
604.4 \\
591.9 \\
588.9 \\
776.6 \\
640.1 \\
674.5 \\
677.9 \\
608.1\end{array}$ & $\begin{array}{l}31.7 \\
21.1 \\
30.0 \\
35.0 \\
25.6 \\
21.7 \\
25.6 \\
32.2 \\
26.7 \\
22.2 \\
27.2 \\
26.7 \\
26.1 \\
26.1 \\
28.3\end{array}$ & $\begin{array}{l}11.8 \\
12.3 \\
11.5 \\
12.7\end{array}$ & $\begin{array}{l}31.4 \\
14.1 \\
23.7 \\
33.4 \\
21.8 \\
15.7 \\
22.5 \\
26.4 \\
22.9 \\
14.4 \\
22.9 \\
23.5 \\
22.7 \\
23.1 \\
26.3\end{array}$ & $\begin{array}{l}4.5 \\
4.4 \\
5.6 \\
4.8 \\
4.3 \\
4.2 \\
4.2 \\
5.6 \\
4.6 \\
4.8 \\
4.8 \\
4.3 \\
4.3 \\
4.1 \\
4.3\end{array}$ & $\begin{array}{l}38.5 \\
21.2 \\
31.0 \\
40.5 \\
28.9 \\
22.8 \\
29.6 \\
33.6 \\
30.0 \\
21.6 \\
30.0 \\
30.6 \\
29.8 \\
30.2 \\
33.4\end{array}$ \\
\hline
\end{tabular}

\section{Doddridge County}

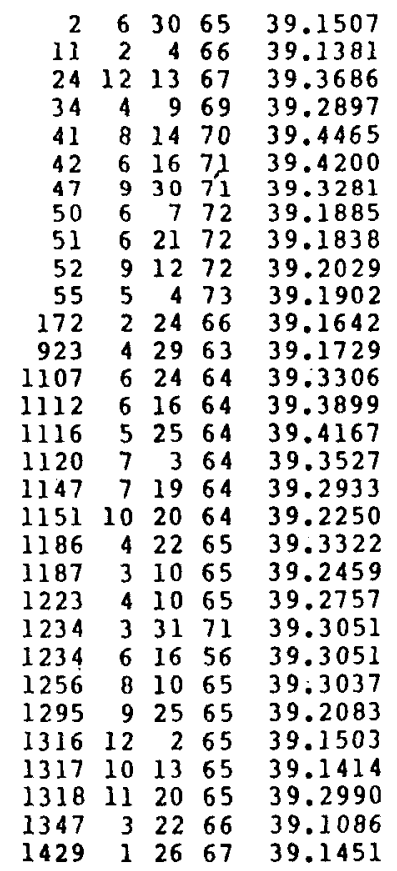

80.7819 80.7854 80.6089 80.6729 80.5951 80.5824 80.5929 80.5747 80.5825 80.5894 80.5996 80.7851 80.7697 80.7114 80.6135 80.5833 80.6236 80.8418 80.6773 80.7146 80.5791 80.6989 80.7350 80.7350 80.6933 80.7046 80.7819 80.7842 80.7433 80.7889 80.7868 $\begin{array}{lll}325.5 & 325.5 & 637.6\end{array}$ $\begin{array}{lll}301.8 & 301.8 & 762.9\end{array}$ $\begin{array}{lll}284.4 & 285.0 & 618.7\end{array}$

$\begin{array}{lll}358.1 & 358.7 & 642.8\end{array}$

$380.4 \quad 381.0 \quad 916.2$

$\begin{array}{llll}318.8 & 319.4 & 670.6\end{array}$

$\begin{array}{lll}311.2 & 311.8 & 716.3\end{array}$

$\begin{array}{lll}321.3 & 321.9 & 701.0\end{array}$

$\begin{array}{lll}352.7 & 353.3 & 731.5\end{array}$

$274.6 \quad 275.2 \quad 760.2$

$291.1 \quad 291.1 \quad 457.2$

$302.4 \quad 303.0 \quad 580.0$

$290.5 \quad 291.1 \quad 613.9$

$284.4 \quad 285.0 \quad 621.8$

$\begin{array}{llll}305.7 & 324.6 & 652.6\end{array}$

$\begin{array}{lll}307.8 & 308.5 & 629.7\end{array}$

$344.1 \quad 344.4 \quad 691.3$

$\begin{array}{llll}341.7 & 342.3 & 698.6\end{array}$

$\begin{array}{lll}316.4 & 317.3 & 649.5\end{array}$

$280.1 \quad 280.1 \quad 835.8$

$324.9 \quad 324.9 \quad 571.2$

$\begin{array}{lll}364.5 & 364.8 & 671.5\end{array}$

$298.4 \quad 299.0 \quad 542.5$

$\begin{array}{llll}338.3 & 338.9 & 639.5\end{array}$

$\begin{array}{llll}279.8 & 280.7 & 658.4\end{array}$

$\begin{array}{llll}344.7 & 344.7 \quad 1485.6\end{array}$

$\begin{array}{llll}322.2 & 322.2 & 609.9\end{array}$

$275.8 \quad 276.5 \quad 585.2$

$\begin{array}{lll}320.3 & 321.3 & 655.3 \\ 266.1 & 266.1 & 553.5\end{array}$ $\begin{array}{lll}22.8 & 11.5 & 17.6\end{array}$ $\begin{array}{lll}23.9 & 11.8 & 15.9\end{array}$ $26.7 \quad 11.7 \quad 24.2$ $30.011 .1 \quad 29.5$ $29.410 .7 \quad 20.5$ $25.6 \quad 11.3 \quad 21.2$ $\begin{array}{lll}28.9 & 11.5 & 24.3\end{array}$ $\begin{array}{lll}25.6 & 11.3 & 20.3\end{array}$ $23.3 \quad 11.5 \quad 16.8$ $21.711 .2 \quad 14.3$ $21.7 \quad 12.0 \quad 12.7$ $21.7 \quad 11.9 \quad 21.4$ $27.8 \quad 11.7 \quad 27.7$ $22.211 .7 \quad 17.2$ $\begin{array}{lll}30.6 & 11.7 & 30.4\end{array}$ $26.711 .3 \quad 23.6$ $26.711 .5 \quad 24.1$ $23.911 .2 \quad 28.4$ $23.3 \quad 11.3 \quad 17.2$ $26.711 .4 \cdot 23.5$ 31.2 .11 .923 .0 19.411 .414 .1 $24.411 .0 \quad 20.0$ $27.8 \quad 11.6 \quad 29.8$ $27.211 .2 \quad 25.0$ $22.8 \quad 11.9 \quad 16.5$ $23.311 .4 \quad 8.1$ $18.911 .6 \quad 12.0$ $22.811 .9 \quad 18.7$ $\begin{array}{llll}23.3 & 11.6 & 17.9\end{array}$ $22.812 .3 \quad 19.2$

$\begin{array}{rr}4.5 & 24.7 \\ 5.5 & 23.1 \\ 4.4 & 31.3 \\ 4.6 & 36.6 \\ 6.7 & 27.8 \\ 4.8 & 28.4 \\ 5.1 & 31.5 \\ 5.0 & 27.5 \\ 5.0 & 24.0 \\ 5.3 & 21.5 \\ 5.5 & 19.9 \\ 3.1 & 28.2 \\ 4.1 & 34.7 \\ 4.3 & 24.2 \\ 4.4 & 37.4 \\ 4.6 & 30.7 \\ 4.5 & 31.2 \\ 5.0 & 25.5 \\ 5.0 & 24.4 \\ 4.6 & 30.6 \\ 6.1 & 30.3 \\ 4.0 & 21.1 \\ 4.8 & 27.2 \\ 3.8 & 36.7 \\ 4.5 & 32.1 \\ 4.7 & 23.6 \\ 10.7 & 15.3 \\ 4.3 & 19.1 \\ 4.1 & 25.7 \\ 4.7 & 25.0 \\ 3.9 & 26.2 \\ 4 & \end{array}$


Doddridge County (cont)

\begin{tabular}{|c|c|c|c|c|c|c|c|c|c|c|c|c|c|}
\hline $\begin{array}{c}\text { Wlili, } \\
\text { III) }\end{array}$ & & $\begin{array}{ll}\mathrm{Mn} \\
1,0\end{array}$ & & $\begin{array}{l}\text { LAT ITUDE } \\
\text { DEGREES }\end{array}$ & $\begin{array}{l}\text { LONGITUDE } \\
\text { DEGREES }\end{array}$ & $\begin{array}{c}\text { FI.EV } \\
M\end{array}$ & $\underset{M}{\text { KBELV }}$ & $\underset{M}{\text { DEPTH }}$ & $\begin{array}{c}\text { BHT } \\
\text { C }\end{array}$ & $\underset{\mathrm{C}}{\text { STEMP }}$ & $\begin{array}{c}\text { GRADI ENT } \\
\text { C/KM }\end{array}$ & $\begin{array}{c}\text { BHTCOR } \\
\text { C }\end{array}$ & $\begin{array}{l}\text { AAPGRAD } \\
\text { C/KM }\end{array}$ \\
\hline $\begin{array}{l}1443 \\
1477 \\
1507 \\
1522 \\
1532 \\
1532 \\
1533 \\
1534 \\
1535 \\
1544 \\
1547 \\
1548 \\
1549 \\
1550\end{array}$ & $\begin{array}{r}3 \\
2 \\
7 \\
10 \\
6 \\
3 \\
11 \\
12 \\
12 \\
2 \\
5 \\
3 \\
6 \\
7\end{array}$ & $\begin{array}{r}17 \\
14 \\
1 \\
12 \\
17 \\
17 \\
12 \\
10 \\
6 \\
11 \\
2 \\
3 \\
12 \\
24\end{array}$ & $\begin{array}{l}67 \\
68 \\
68 \\
68 \\
69 \\
69 \\
68 \\
68 \\
68 \\
69 \\
69 \\
72 \\
69 \\
69\end{array}$ & $\begin{array}{l}39.4007 \\
39.3642 \\
39.2771 \\
39.1428 \\
39.1103 \\
39.1103 \\
39.1101 \\
39.1299 \\
39.1194 \\
39.1071 \\
39.1325 \\
39.2803 \\
39.2957 \\
39.3012\end{array}$ & $\begin{array}{l}80.7146 \\
80.6061 \\
80.7433 \\
80.7882 \\
80.7976 \\
80.7976 \\
80.7979 \\
80.8038 \\
80.8109 \\
80.7847 \\
80.7995 \\
80.7021 \\
80.7461 \\
80.7376\end{array}$ & $\begin{array}{l}262.7 \\
348.1 \\
333.5 \\
350.8 \\
347.5 \\
347.5 \\
294.4 \\
379.5 \\
348.4 \\
260.3 \\
372.2 \\
268.8 \\
273.7 \\
295.4\end{array}$ & $\begin{array}{l}264.6 \\
348.7 \\
333.8 \\
351.4 \\
347.5 \\
348.1 \\
295.4 \\
380.4 \\
349.0 \\
260.9 \\
372.8 \\
269.4 \\
274.3 \\
296.0\end{array}$ & $\begin{array}{l}637.3 \\
690.1 \\
620.6 \\
823.6 \\
608.1 \\
667.8 \\
598.6 \\
652.9 \\
827.8 \\
771.4 \\
611.7 \\
530.7 \\
592.2 \\
623.9\end{array}$ & $\begin{array}{l}26.7 \\
26.7 \\
27.2 \\
29.4 \\
15.6 \\
19.4 \\
18.3 \\
20.0 \\
25.0 \\
23.3 \\
20.0 \\
22.2 \\
25.0 \\
29.4\end{array}$ & $\begin{array}{l}11.9 \\
11.1 \\
11.3 \\
11.3 \\
11.4 \\
11.4 \\
11.9 \\
11.0 \\
11.3 \\
12.2 \\
11.1 \\
12.0 \\
11.9 \\
11.7\end{array}$ & $\begin{array}{r}23.2 \\
22.6 \\
25.6 \\
22.0 \\
6.9 \\
12.1 \\
10.8 \\
13.8 \\
16.5 \\
14.4 \\
14.6 \\
19.4 \\
22.1 \\
28.5\end{array}$ & $\begin{array}{l}4.8 \\
4.2 \\
4.7 \\
6.0 \\
5.6 \\
4.3 \\
3.7 \\
4.2 \\
4.4\end{array}$ & $\begin{array}{l}30.3 \\
29.7 \\
32.7 \\
29.3 \\
14.0 \\
19.2 \\
17.8 \\
20.9 \\
23.7 \\
21.6 \\
21.6 \\
26.3 \\
29.2 \\
35.6\end{array}$ \\
\hline
\end{tabular}

Fayette County

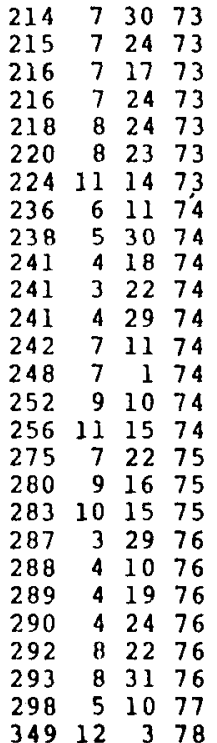

38.1792

38.1647

38.2971

38.1971

38.2006

38.0993

38.1734

38.1970

38.2069

38.1134

38.1134

38.1134

38.1315

38.0836

37.9942

38.1582

38.1761

38.2382

38.1771

38.0822

38.0734

38.0785

38.1595

38.0704

38.0776

38.1894

$\begin{array}{lllll}349 & 12 & 3 & 78 & 38.0605\end{array}$

$667.2 \quad 669.3 \quad 969.3$
81.2037

81.0898

81.2605

B1.2605

81.2400

81.2800

81.1820

81.2341

81.2320

80.9851

80.9851

80.9851

81.1761

81.3695

81.1646

81.1643

81.2559

81.2195

80.9642

81.3069

81.3003

81.2960

80.9612

81.2575

81.1937

81.1482

81.0270
$326.1 \cdot 326.7 \quad 710.5$ $326.2 \quad 330.7 \quad 760.2$ $\begin{array}{llll}326.1 & 330.7 \quad 1879.4\end{array}$

$\begin{array}{llll}442.3 & 447.1 & 836.4\end{array}$

$316.4 \quad 318.5 \quad 662.9$

$240.5 \quad 241.1 \quad 577.6$

$\begin{array}{lll}490.7 & 493.8 & 811.7\end{array}$

$\begin{array}{llll}471.8 & 474.9 & 819.0\end{array}$

$702.6 \quad 707.42416 .5$

$\begin{array}{llll}702.6 & 707.4 & 739.4\end{array}$

$\begin{array}{lll}702.6 & 707.4 & 2642.6\end{array}$

$\begin{array}{lll}315.5 & 316.4 & 638.6\end{array}$

$\begin{array}{llll}478.8 & 482.2 & 789.4\end{array}$

$\begin{array}{llll}634.3 & 637.3 & 1043.9\end{array}$

$338.3 \quad 340.8 \quad 609.0$

$\begin{array}{lll}471.8 & 474.3 & 853.4\end{array}$

$338.6 \quad 341.7 \quad 697.1$

$\begin{array}{lll}555.3 & 557.8 & 817.8\end{array}$

$\begin{array}{llll}470.6 & 472.7 & 803.8\end{array}$

$\begin{array}{llll}470.9 & 473.0 & 801.6\end{array}$

$518.8 \quad 521.8 \quad 645.3$

$\begin{array}{lll}365.8 & 367.9 & 708.4\end{array}$

$539.2 \quad 541.3 \quad 860.5$

435.9

605.9609 .0666 .0 $\begin{array}{lll}420.3 & 420.9 & 847.0\end{array}$ $\begin{array}{lll}27.2 & 12.6 \quad 20.5\end{array}$

$26.7 \quad 11.7 \quad 17.6$ $32.8 \quad 12.6 \quad 26.6$

$32.2 \quad 11.4 \quad 24.9$

$29.412 .8 \quad 25.1$ $29.4 \quad 13.5 \quad 27.6$

$2.211 .0 \quad 26.2$

$32.211 .2 \quad 25.7$

$\begin{array}{lll}74.4 & 9.0 & 27.1\end{array}$

$27.8 \quad 9.0 \quad 25.4$

$\begin{array}{lll}73.3 & 9.0 & 24.3\end{array}$

$\begin{array}{lll}23.9 & 12.8 & 17.4\end{array}$

$31.711 .2 \quad 25.9$

$\begin{array}{lll}30.6 & 9.8 & 19.9\end{array}$

$31.111 .2 \quad 23.3$

$\begin{array}{lll}25.6 & 12.4 & 18.8\end{array}$

$\begin{array}{lll}30.6 & 10.4 & 24.7\end{array}$

$\begin{array}{lll}26.7 & 11.3 & 19.1\end{array}$

$31.7 \quad 9.4 \quad 23.0$

$28.911 .3 \quad 21.9$

$28.9 \quad 10.8 \quad 28.1$

$32.2 \quad 12.4 \quad 28.0$

$29.410 .7 \quad 21.8$

$\begin{array}{lll}23.3 & 11.5 & 15.7\end{array}$

$27.210 .0 \quad 25.8$ $\begin{array}{lll}3.9 & 12.6 & 27.3\end{array}$

$\begin{array}{lll}26.1 & 12.5 & 22.3\end{array}$

$\begin{array}{rr}5.1 & 27.7 \\ 6.1 & 24.9 \\ 5.5 & 33.8 \\ 13.2 & 34.3 \\ 6.1 & 32.1 \\ 4.7 & 32.2 \\ 4.1 & 34.7 \\ 5.9 & 33.4 \\ 5.9 & 33.0 \\ 15.9 & 33.7 \\ 5.3 & 32.6 \\ 16.9 & 30.7 \\ 4.5 & 24.5 \\ 5.7 & 33.1 \\ 7.6 & 27.1 \\ 4.3 & 29.4 \\ 6.2 & 30.6 \\ 5.0 & 26.0 \\ 5.9 & 31.9 \\ 5.8 & 26.3 \\ 7.1 & 30.2 \\ 5.8 & 29.1 \\ 4.6 & 35.2 \\ 5.1 & 35.2 \\ 6.2 & 29.1 \\ 5.4 & 23.0 \\ 4.8 & 33.0\end{array}$

\section{Gilmer County}

$\begin{array}{rrrrr}2010 & 9 & 2 & 69 & 38.8369 \\ 2015 & 8 & 15 & 69 & 39.0658 \\ 2045 & 3 & 14 & 70 & 38.8445 \\ 2049 & 4 & 5 & 70 & 38.9863 \\ 2057 & 4 & 11 & 70 & 38.9300 \\ 2058 & 4 & 25 & 70 & 38.8996 \\ 2062 & 6 & 2 & 70 & 38.9885 \\ 2063 & 8 & 13 & 70 & 38.9846 \\ 2166 & 9 & 9 & 71 & 39.0548 \\ 2223 & 7 & 23 & 72 & 39.0605 \\ 2231 & 8 & 19 & 72 & 38.9865 \\ 2233 & 7 & 13 & 72 & 39.0664 \\ 2238 & 7 & 30 & 72 & 39.0372 \\ 2255 & 9 & 21 & 72 & 38.8594 \\ 2328 & 5 & 31 & 73 & 38.8148 \\ 2333 & 8 & 3 & 73 & 39.0656 \\ 2451 & 6 & 25 & 74 & 38.8205 \\ 2486 & 7 & 21 & 74 & 38.8228\end{array}$

80.9731 80.8059 80.7976

80.8754

80.9477

80.9565

80.8683

80.8641

80.7731

80.7 .933

80.8946

80.7446

80.8556

80.9533

81.0252

80.7763

80.9274
81.0048

$248.7 \quad 249.3 \quad 1269.5$

$\begin{array}{lll}326.4 & 328.3 & 739.7 \\ 322.2 & 323.1 & 715.4\end{array}$

$286.5 \quad 287.1 \quad 624.8$

$215.8 \quad 217.0 \quad 585.2$

$331.3 \quad 333.5 \quad 625.8$

$291.1 \quad 291.7 \quad 642.2$

277.4278 .0

624.2

$\begin{array}{lll}323.1 & 324.9 & 665.4 \\ 338.3 & 339.9 & 688.2\end{array}$

688.2

$\begin{array}{lll}288.6 & 289.3 & 553.5 \\ 261.2 & 263.0 & 589.2\end{array}$

$316.7 \quad 317.9 \quad 630.0$

$\begin{array}{lll}328.9 & 330.7 & 657.1\end{array}$

$\begin{array}{rrr}305.4 & 307.2 & 649.5 \\ 244.1 & 247.5 & 1467.9\end{array}$

$231.6 \quad 233.2 \quad 539.5$

$339.9 \quad 343.2 \quad 753.2$
8.5

5.3

5.1

4.4

4.1

4.4

4.6
4.4

4.7

4.9

3.9

4.2

4.5
4.7

4.6

10.6

3.8

5.4
30.2

35.1

27.1

26.0

26.9

33.3

25.8

38.1

35.7

29.4

33.4

1.7

27.9

25.6

30.7 


\section{Gilmer County (cont)}

\begin{tabular}{|c|c|c|c|c|c|c|c|c|c|c|c|c|c|}
\hline $\begin{array}{c}\text { Wh:I, } \\
\text { N1O }\end{array}$ & & $\begin{array}{l}\mathrm{DN} \\
\mathrm{LO}\end{array}$ & & $\begin{array}{l}\text { LATITUDF: } \\
\text { DEGREES }\end{array}$ & $\begin{array}{l}\text { I,ONG ITUDE } \\
\text { DEGREES }\end{array}$ & $\begin{array}{c}\text { FI.EV } \\
\text { M }\end{array}$ & $\begin{array}{c}\text { KBELV } \\
M\end{array}$ & $\underset{\mathrm{M}}{\text { DEPTH }}$ & $\underset{\mathrm{C}}{\mathrm{BHT}}$ & $\begin{array}{c}\text { STEMP } \\
\mathrm{C}\end{array}$ & $\begin{array}{c}\text { GRADIENT } \\
\mathrm{C} / \mathrm{KM}\end{array}$ & C & $\begin{array}{l}\text { AAPGRAD } \\
\mathrm{C} / \mathrm{KM}\end{array}$ \\
\hline $\begin{array}{l}2486 \\
2539 \\
2546 \\
2559 \\
2670 \\
2685 \\
3024 \\
3029 \\
3211\end{array}$ & $\begin{array}{l}7 \\
7 \\
6 \\
6 \\
7 \\
9 \\
8 \\
6 \\
9 \\
8\end{array}$ & $\begin{array}{r}27 \\
9 \\
3 \\
14 \\
22 \\
30 \\
21 \\
10 \\
13 \\
8\end{array}$ & $\begin{array}{l}74 \\
75 \\
75 \\
67 \\
76 \\
76 \\
78 \\
78 \\
78 \\
78\end{array}$ & $\begin{array}{l}38.8228 \\
38.8990 \\
38.8935 \\
38.9753 \\
38.9023 \\
38.9664 \\
39.0575 \\
38.8808 \\
38.9080 \\
38.9178\end{array}$ & $\begin{array}{l}81.0048 \\
80.6255 \\
80.9709 \\
80.7673 \\
80.6380 \\
80.8641 \\
80.8877 \\
80.9473 \\
80.8866 \\
80.8563\end{array}$ & $\begin{array}{l}339.9 \\
373.1 \\
256.6 \\
291.1 \\
371.2 \\
226.5 \\
361.5 \\
306.6 \\
332.2 \\
272.2\end{array}$ & $\begin{array}{l}343.2 \\
374.6 \\
258.2 \\
291.7 \\
372.8 \\
228.6 \\
364.5 \\
308.2 \\
335.3 \\
275.2\end{array}$ & $\begin{array}{r}1892.2 \\
716.0 \\
564.5 \\
526.1 \\
714.5 \\
506.9 \\
1630.7 \\
623.0 \\
1600.8 \\
1573.4\end{array}$ & $\begin{array}{l}56.7 \\
23.3 \\
22.2 \\
20.6 \\
21.7 \\
27.2 \\
45.0 \\
26.7 \\
43.3 \\
42.2\end{array}$ & $\begin{array}{ll}7 & 11.7 \\
3 & 11.3 \\
2 & 12.5 \\
6 & 12.1 \\
7 & 11.3 \\
2 & 12.7 \\
0 & 11.3 \\
7 & 12.0 \\
3 & 11.7 \\
2 & 12.3\end{array}$ & $\begin{array}{l}23.8 \\
16.8 \\
17.3 \\
16.2 \\
14.5 \\
28.7 \\
20.7 \\
23.5 \\
19.8 \\
19.0\end{array}$ & $\begin{array}{r}13.2 \\
5.1 \\
4.0 \\
3.7 \\
5.1 \\
3.5 \\
11.7 \\
4.4 \\
11.5 \\
11.3\end{array}$ & $\begin{array}{l}.8 \\
.0 \\
.3 \\
.1 \\
.6 \\
.6 \\
.8 \\
.6 \\
.9 \\
.2\end{array}$ \\
\hline
\end{tabular}

\section{Grant County}

$\begin{array}{rrrrrrrrrrrrrr}2 & 10 & 24 & 65 & 39.2239 & 79.1202 & 716.0 & 719.9 & 3960.6 & 87.2 & 7.5 & 20.1 & 18.7 & 24.9 \\ 2 & 10 & 6 & 65 & 39.2239 & 79.1202 & 716.0 & 719.9 & 3054.4 & 70.0 & 7.5 & 20.5 & 18.1 & 26.4\end{array}$

Greenbrier County

$\begin{array}{rrrrrrrrrrrrrr}12 & 6 & 24 & 60 & 38.1412 & 80.4738 & 1125.6 & 1129.9 & 2488.1 & 62.8 & 4.8 & 23.3 & 16.3 & 29.8 \\ 14 & 6 & 20 & 63 & 37.7599 & 80.5222 & 632.8 & 636.1 & 2164.1 & 64.4 & 10.1 & 25.1 & 14.7 & 31.9 \\ 16 & 11 & 22 & 63 & 38.0912 & 80.8142 & 840.6 & 844.3 & 2437.2 & 73.3 & 7.7 & 26.9 & 16.0 & 33.5 \\ 18 & 1 & 30 & 68 & 38.0927 & 80.8448 & 810.8 & 815.3 & 2665.5 & 71.7 & 8.0 & 23.9 & 17.0 & 30.3 \\ 19 & 12 & 10 & 69 & 37.8865 & 80.5310 & 782.7 & 786.4 & 2356.7 & 60.0 & 8.5 & 21.9 & 15.7 & 28.5 \\ 20 & 3 & 5 & 70 & 37.9272 & 80.5473 & 644.3 & 648.0 & 2471.6 & 71.7 & 9.8 & 25.0 & 16.2 & 31.6 \\ 21 & 6 & 20 & 74 & 37.9640 & 80.6269 & 876.3 & 879.3 & 1033.3 & 30.6 & 7.5 & 22.3 & 7.5 & 29.6 \\ 22 & 11 & 16 & 75 & 38.0608 & 80.7328 & 1055.2 & 1060.1 & 909.8 & 32.2 & 5.6 & 29.3 & 6.6 & 36.6 \\ 22 & 12 & 7 & 75 & 38.0608 & 80.7328 & 1055.2 & 1060.1 & 2532.0 & 61.7 & 5.6 & 22.1 & 16.4 & 28.6 \\ 22 & 1 & 6 & 76 & 38.0608 & 80.7328 & 1055.2 & 1060.1 & 3084.6 & 94.4 & 5.6 & 28.8 & 18.2 & 34.7 \\ 23 & 4 & 3 & 77 & 38.1750 & 80.5094 & 1018.9 & 1023.5 & 723.9 & 33.3 & 5.8 & 38.0 & 5.2 & 45.2\end{array}$

Hampshire County

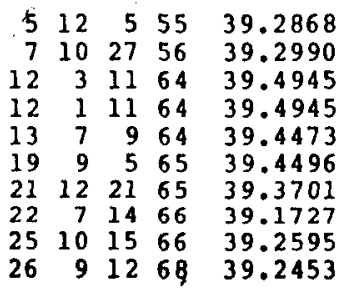

78.6620

78.6790

78.6363

78.6363

78.5467

78.5409

78.6000

78.7711

78.6819

78.6925

\begin{abstract}
$\begin{array}{llll}390.4 & 392.0 & 1637.1 \\ 409.0 & 412.4 & 1857.1\end{array}$
$\begin{array}{llll}409.0 & 412.4 & 1857.1 \\ 285.9 & 292.3 & 4262.9\end{array}$

$285.9 \quad 292.3 \quad 2663.6$

$276.8 \quad 280.41653 .5$

$207.0 \quad 210.6 \quad 1707.2$

$328.3 \quad 331.91710 .5$

$534.9 \quad 539.22073 .6$

$438.0 \quad 438.0 \quad 1626.1$

$477.0 \quad 482.2 \quad 1825.8$
\end{abstract}

$60.010 .5 \quad 30.2$

$51.110 .3 \quad 22.0$

$82.211 .3 \quad 16.6$

$51.7 \quad 11.3 \quad 15.1$

$37.2 \quad 11.5 \quad 15.6$

44.412 .218 .9

$57.211 .0 \quad 27.0$

$58.9 \quad 9.2 \quad 23.9$

$41.1 \quad 10.1 \quad 19.1$

$\begin{array}{lll}41.1 & 9.7 & 17.2\end{array}$
11.7

13.0

18.1

17.0

11.8

12.1

12.2

14.3

11.6

12.9
37.4

29.0

20.9

21.5

22,7

26.0

34.1

30.8

26.2

24.2

\section{Hancock County}

$\begin{array}{rrrrr}80 & 5 & 23 & 65 & 40.5400 \\ 80 & 8 & 9 & 65 & 40.5400 \\ 87 & 3 & 1 & 75 & 40.5514\end{array}$

80.556

0.5332

316.7

$320.6 \quad 1742.2$

$40.6 \quad 10.2 \quad 17.4$ $\begin{array}{llllll}316.7 & 320.6 & 3163.8 & 78.9 & 10.2 & 21.7\end{array}$ $\begin{array}{llllll}394.1 & 397.8 & 2340.6 & 60.0 & 9.4 & 21.6\end{array}$
12.4

$18.4 \quad 24.5$

15.6

\section{Hardy County}

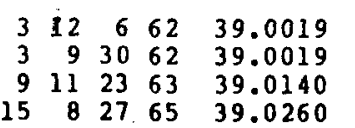

78.8503 78.8503

78.8412

78.8200
598.9
598.9

626.4

626.4
602.33226 .6 602.32304 .0 630.0
607.5
62286.0 $\begin{array}{lll}70.6 & 8.8 & 19.1\end{array}$

$\begin{array}{lll}48.3 \quad 8.8 & 17.2\end{array}$

$51.1 \quad 8.5 \quad 19.9$

$60.0 \quad 8.7 \quad 22.4$ $\begin{array}{ll}18.5 & 24.9 \\ 15.4 & 23.9 \\ 14.6 & 26.7\end{array}$ 
Harrison County

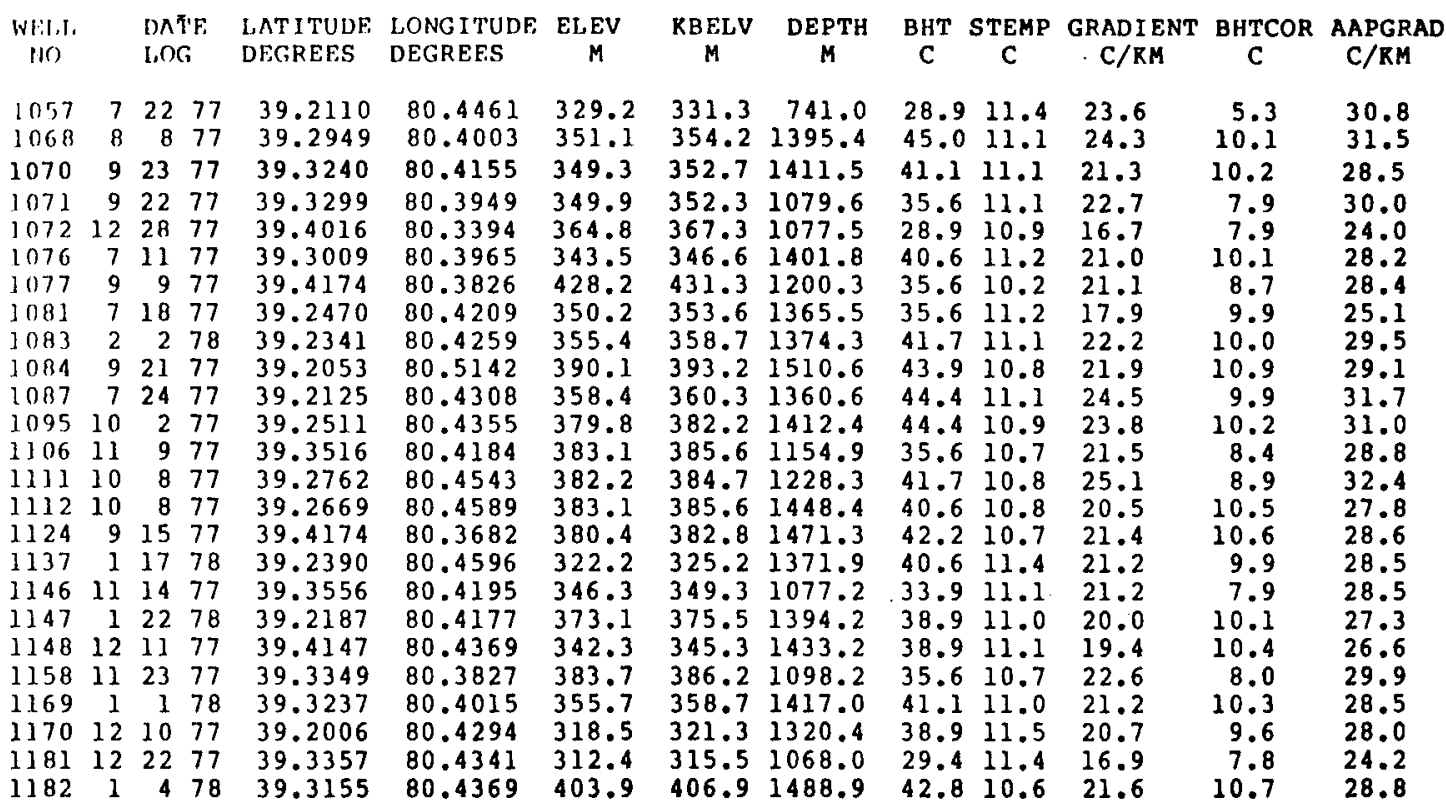

\section{Jackson County}

$\begin{array}{rrrrr}1145 & 7 & 2 & 67 & 38.7740 \\ 1158 & 9 & 11 & 68 & 38.9226 \\ 1182 & 12 & 14 & 69 & 38.8995 \\ 1183 & 11 & 21 & 69 & 38.8254 \\ 1191 & 2 & 19 & 70 & 38.8618 \\ 1201 & 4 & 13 & 70 & 38.8892 \\ 1201 & 4 & 17 & 70 & 38.8892 \\ 1203 & 5 & 12 & 70 & 38.9618 \\ 1212 & 6 & 22 & 70 & 38.9739 \\ 1212 & 8 & 10 & 70 & 38.8629 \\ 1216 & 7 & 14 & 70 & 38.8895 \\ 1219 & 7 & 16 & 70 & 38.8910 \\ 1226 & 8 & 29 & 70 & 38.8456 \\ 1246 & 12 & 19 & 70 & 39.0578 \\ 1247 & 12 & 4 & 70 & 38.9012 \\ 1248 & 12 & 2 & 70 & 38.8659 \\ 1267 & 5 & 2 & 71 & 38.8675 \\ 1282 & 6 & 9 & 71 & 38.8951 \\ 1293 & 7 & 24 & 71 & 38.8475 \\ 1365 & 3 & 22 & 74 & 38.8062 \\ 1386 & 1 & 13 & 77 & 38.7166 \\ 1388 & 12 & 23 & 76 & 38.9643 \\ 1389 & 6 & 3 & 77 & 38.9643 \\ 1391 & 6 & 6 & 77 & 38.8417 \\ 1425 & 1 & 30 & 78 & 38.8715\end{array}$
81.8018 81.6995 81.5787 81.7343 81.7315 81.7196 81.7196 81.5920 81.6354 81.7203 81.7436 81.7342 81.7444 81.7059 81.7425 81.7613 81.7713 81.7892 81.7810 81.8064 81.7279 81.6808 81.6808 81.8237 81.8239
250.5 225.6 $193.5 \quad 715.1$ 210.6214 .91698 .0 $225.6 \quad 230.1 \quad 1699.9$ $\begin{array}{lll}259.1 & 263.7 & 1715.4\end{array}$ $259.1 \quad 263.7 \quad 1729.4$ $318.2 \quad 322.8 \quad 1682.2$ $\begin{array}{lll}189.0 & 193.5 & 1709.6\end{array}$ $265.2 \quad 269.7 \quad 1755.0$ 241.1245 .11692 .9 $\begin{array}{llll}259.1 & 263.7 & 1714.8\end{array}$ $253.6 \quad 257.9 \quad 1737.4$ $224.0 \quad 227.7 \quad 1829.1$ $230.7 \quad 235.0 \quad 1929.4$ $260.6 \quad 265.2 \quad 1680.4$ $261.5 \quad 264.9 \quad 1660.2$ $248.4 \quad 253.6 \quad 1613.3$ $261.5 \quad 266.7 \quad 1666.6$ $\begin{array}{llll}253.9 & 257.6 & 1373.7\end{array}$ 204.8204 .81691 .0 $215.2 \quad 217.6 \quad 1441.1$ $219.8 \quad 222.2 \quad 1413.1$ $234.4 \quad 236.8 \quad 1426.8$ $196.6200 .3 \quad 1606.0$ $\begin{array}{lll}50.0 & 12.6 & 21.2\end{array}$ $\begin{array}{lll}53.3 & 12.7 & 24.3\end{array}$ $21.113 .1 \quad 11.2$ 48.912 .921 .2 $44.412 .8 \quad 18.6$ $\begin{array}{llll}46.7 & 12.4 & 20.0\end{array}$ $\begin{array}{llll}47.8 & 12.4 & 20.5\end{array}$ $47.811 .7 \quad 21.4$ $\begin{array}{lll}51.1 & 13.0 & 22.3\end{array}$ $\begin{array}{lll}53.3 & 12.4 & 23.3\end{array}$ 48.312 .621 .1 $\begin{array}{lll}47.2 & 12.4 \quad 20.3\end{array}$ $\begin{array}{llll}46.7 & 12.5 & 19.7\end{array}$ $\begin{array}{lll}45.6 & 12.6 & 18.0\end{array}$ $60.012 .7 \quad 24.5$ $\begin{array}{llll}43.3 & 12.4 & 18.4\end{array}$ $42.812 .4 \quad 18.3$ $\begin{array}{lll}46.1 & 12.5 & 20.8\end{array}$ $\begin{array}{lll}47.8 & 12.4 & 21.2\end{array}$ $\begin{array}{lll}41.7 & 12.5 & 21.2\end{array}$ $52.213 .2 \quad 23.1$ $\begin{array}{llll}42.8 & 12.8 & 20.8\end{array}$ $\begin{array}{llll}37.8 & 12.7 & 17.7\end{array}$ $51.112 .7 \quad 26.9$

$\begin{array}{ll}12.5 & 28.2 \\ 11.9 & 31.4 \\ 5.1 & 18.4 \\ 12.1 & 28.3 \\ 12.1 & 25.8 \\ 12.2 & 27.1 \\ 12.3 & 27.6 \\ 12.0 & 28.5 \\ 12.1 & 29.4 \\ 12.4 & 30.4 \\ 12.0 & 28.5 \\ 12.2 & 27.4 \\ 12.3 & 26.8 \\ 12.9 & 25.1 \\ 13.5 & 31.5 \\ 12.0 & 25.5 \\ 11.8 & 25.4 \\ 11.5 & 28.0 \\ 11.9 & 28.3 \\ 10.0 & 28.4 \\ 12.0 & 30.2 \\ 10.4 & 28.1 \\ 10.2 & 25.0 \\ 10.3 & 34.1 \\ 11.5 & 27.8\end{array}$


Kanawha County

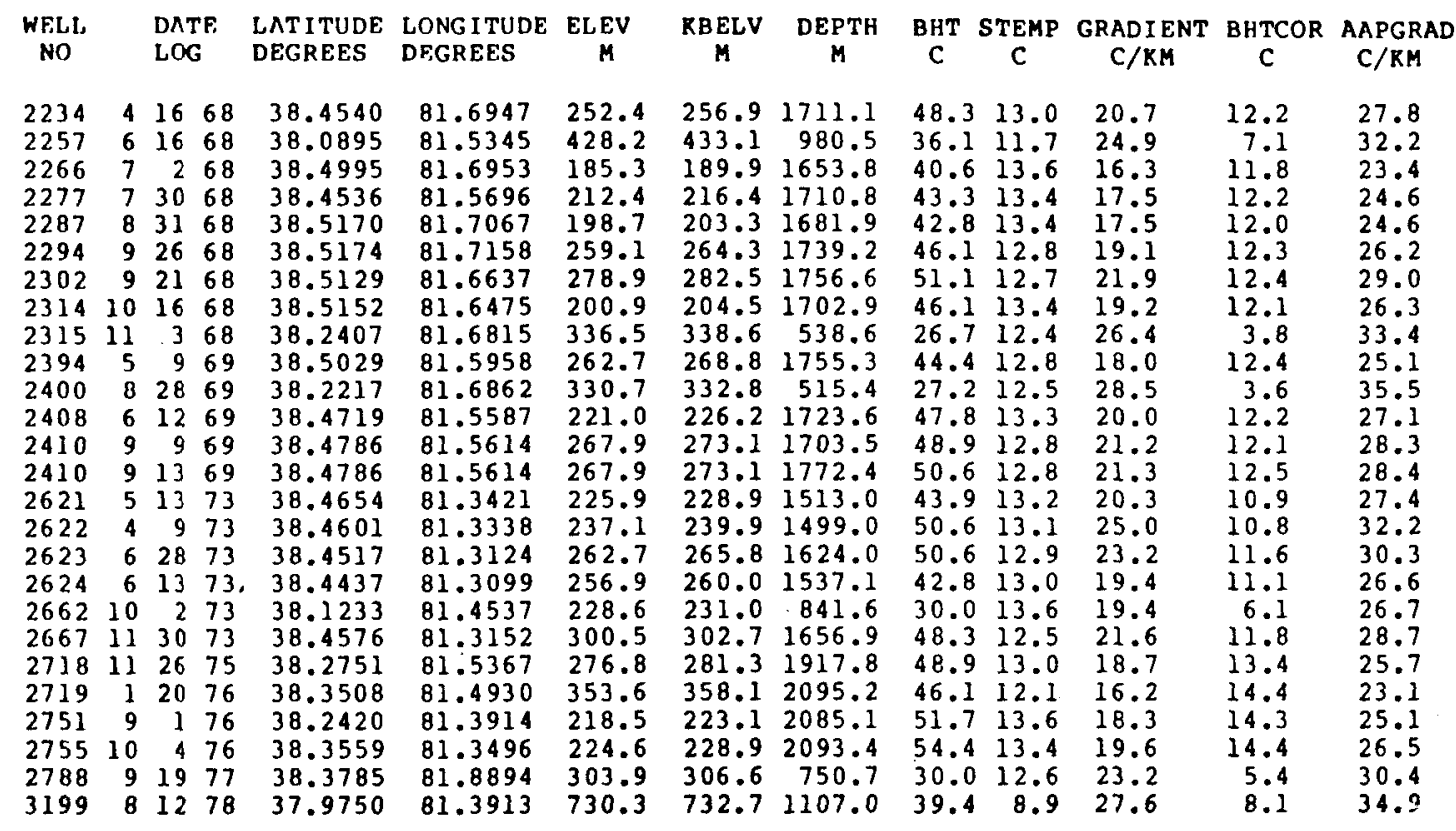

Lewis County

\begin{tabular}{|c|c|c|c|c|c|c|c|c|c|c|c|c|c|}
\hline $\begin{array}{l}1877 \\
1878 \\
1879 \\
1880 \\
1881 \\
1882 \\
1889 \\
1891 \\
1895 \\
1896 \\
1897 \\
1901\end{array}$ & $\begin{array}{r}10 \\
11 \\
11 \\
11 \\
11 \\
11 \\
5 \\
4 \\
3 \\
3 \\
4 \\
6\end{array}$ & $\begin{array}{r}27 \\
5 \\
8 \\
13 \\
12 \\
19 \\
1 \\
24 \\
16 \\
28 \\
6 \\
3\end{array}$ & $\begin{array}{l}72 \\
72 \\
72 \\
72 \\
72 \\
72 \\
73 \\
73 \\
73 \\
73 \\
73 \\
73\end{array}$ & $\begin{array}{l}39.0839 \\
39.0969 \\
39.0465 \\
39.0680 \\
39.0402 \\
39.0221 \\
39.0884 \\
39.0858 \\
38.8953 \\
38.8896 \\
38.9738 \\
39.0807\end{array}$ & $\begin{array}{l}80.4088 \\
80.3645 \\
80.4379 \\
80.3899 \\
80.5103 \\
80.5297 \\
80.5202 \\
80.3917 \\
80.5732 \\
80.5700 \\
80.5459 \\
80.5353\end{array}$ & $\begin{array}{l}356.3 \\
409.3 \\
368.5 \\
352.7 \\
350.8 \\
363.3 \\
349.3 \\
309.1 \\
319.4 \\
280.4 \\
357.5 \\
363.9\end{array}$ & $\begin{array}{l}359.1 \\
412.4 \\
371.6 \\
356.6 \\
353.6 \\
366.4 \\
353.0 \\
309.7 \\
321.3 \\
282.2 \\
359.7 \\
365.8\end{array}$ & $\begin{array}{r}1336.9 \\
1378.6 \\
1385.6 \\
1356.1 \\
1407.9 \\
1500.8 \\
2176.9 \\
1293.0 \\
735.8 \\
705.9 \\
661.4 \\
492.6\end{array}$ & $\begin{array}{l}37.2 \\
38.3 \\
39.4 \\
40.0 \\
41.1 \\
37.8 \\
56.7 \\
40.0 \\
25.0 \\
23.3 \\
21.7 \\
25.6\end{array}$ & $\begin{array}{l}11.3 \\
10.7 \\
11.2 \\
11.3 \\
11.4 \\
11.3 \\
11.3 \\
11.8 \\
11.9 \\
12.2 \\
11.4 \\
11.2\end{array}$ & $\begin{array}{l}19.4 \\
20.0 \\
20.4 \\
21.2 \\
21.1 \\
17.7 \\
20.8 \\
21.8 \\
17.9 \\
15.7 \\
15.5 \\
29.1\end{array}$ & $\begin{array}{r}9.7 \\
10.0 \\
10.0 \\
9.8 \\
10.2 \\
10.8 \\
14.8 \\
9.4 \\
5.3 \\
5.1 \\
4.7 \\
3.4\end{array}$ & $\begin{array}{l}26.7 \\
27.3 \\
27.6 \\
28.4 \\
28.4 \\
24.9 \\
27.6 \\
29.1 \\
25.1 \\
22.9 \\
22.7 \\
36.0\end{array}$ \\
\hline $\begin{array}{l}1904 \\
1907 \\
1909 \\
2101 \\
2105 \\
2120 \\
2148 \\
2173 \\
2176 \\
2184 \\
2490 \\
2540 \\
2548 \\
2554\end{array}$ & $\begin{array}{r}5 \\
5 \\
5 \\
3 \\
9 \\
6 \\
2 \\
4 \\
6 \\
6 \\
8 \\
12 \\
12 \\
1\end{array}$ & $\begin{array}{r}6 \\
28 \\
21 \\
25 \\
24 \\
20 \\
23 \\
14 \\
7 \\
25 \\
30 \\
15 \\
21 \\
9\end{array}$ & $\begin{array}{l}73 \\
73 \\
73 \\
77 \\
77 \\
77 \\
78 \\
78 \\
78 \\
78 \\
78 \\
78 \\
78 \\
79\end{array}$ & $\begin{array}{l}39.0524 \\
39.0638 \\
39.0619 \\
39.0726 \\
38.9305 \\
39.0535 \\
39.0940 \\
39.1220 \\
39.1194 \\
39.1172 \\
39.0357 \\
39.0784 \\
39.0740 \\
39.0284\end{array}$ & $\begin{array}{l}80.3818 \\
80.5283 \\
80.3659 \\
80.3347 \\
80.3743 \\
80.4243 \\
80.5331 \\
80.5433 \\
80.5121 \\
80.5364 \\
80.4897 \\
80.4494 \\
80.4524 \\
80.4925\end{array}$ & $\begin{array}{l}375.2 \\
381.0 \\
339.9 \\
413.6 \\
379.2 \\
403.9 \\
339: 9 \\
378.6 \\
439.8 \\
403.9 \\
406.6 \\
423.4 \\
406.0 \\
354.5\end{array}$ & $\begin{array}{l}378.3 \\
385.3 \\
342.9 \\
416.1 \\
382.2 \\
406.3 \\
342.9 \\
381.9 \\
443.2 \\
407.2 \\
409.7 \\
426.4 \\
409.0 \\
356.9\end{array}$ & $\begin{array}{r}1394.5 \\
2196.1 \\
1332.6 \\
827.2 \\
1326.5 \\
1408.2 \\
1349.0 \\
710.8 \\
1467.0 \\
716.0 \\
1485.0 \\
1407.9 \\
1408.2 \\
1432.9\end{array}$ & $\begin{array}{l}40.0 \\
55.6 \\
39.4 \\
29.4 \\
47.8 \\
43.3 \\
40.0 \\
23.3 \\
37.2 \\
27.8 \\
62.8 \\
38.3 \\
35.0 \\
38.3\end{array}$ & $\begin{array}{l}11.1 \\
11.0 \\
11.5 \\
10.7 \\
11.2 \\
10.8 \\
11.4 \\
11.0 \\
10.4 \\
10.8 \\
10.8 \\
10.6 \\
10.8 \\
11.4\end{array}$ & $\begin{array}{l}20.7 \\
20.3 \\
21.0 \\
22.6 \\
27.6 \\
23.1 \\
21.2 \\
17.3 \\
18.3 \\
23.8 \\
35.0 \\
19.7 \\
17.2 \\
18.8\end{array}$ & $\begin{array}{r}10.1 \\
14.9 \\
9.7 \\
6.0 \\
9.6 \\
10.2 \\
9.8 \\
5.1 \\
10.6 \\
5.1 \\
10.7 \\
10.2 \\
10.2 \\
10.4\end{array}$ & $\begin{array}{l}28.0 \\
27.1 \\
28.3 \\
29.9 \\
34.8 \\
30.3 \\
28.4 \\
24.5 \\
25.5 \\
30.9 \\
42.2 \\
26.9\end{array}$ \\
\hline
\end{tabular}




\section{Lincoln County}

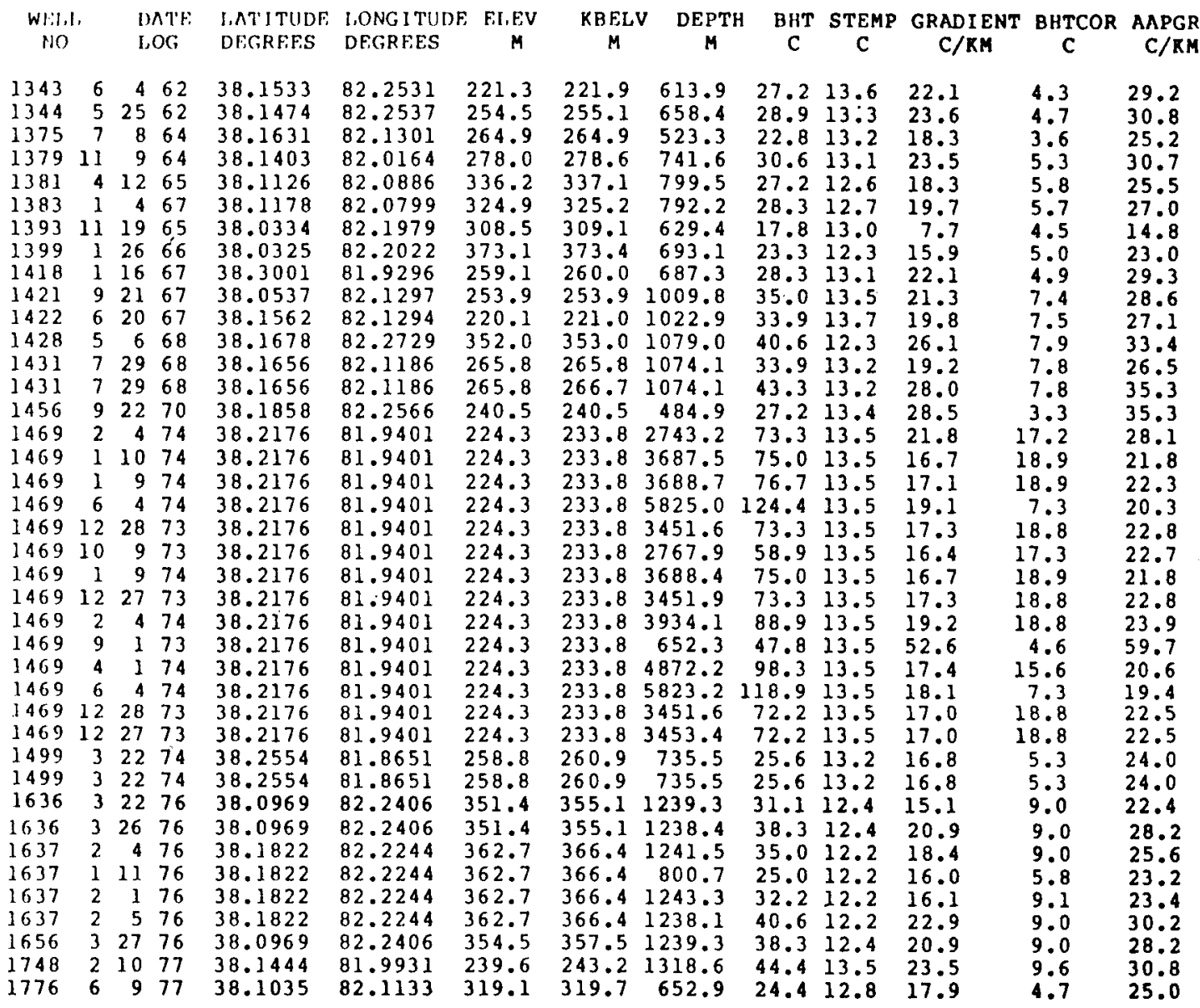

Logan County

$\begin{array}{rrrrr}868 & 7 & 10 & 66 & 37.8247 \\ 871 & 8 & 28 & 67 & 37.9580 \\ 873 & 6 & 18 & 67 & 37.9226 \\ 874 & 7 & 12 & 67 & 37.8072 \\ 881 & 1 & 28 & 69 & 38.0048 \\ 882 & 7 & 9 & 69 & 37.9530 \\ 889 & 9 & 15 & 70 & 37.8007 \\ 892 & 12 & 22 & 70 & 37.7725 \\ 916 & 5 & 16 & 72 & 37.8486 \\ 917 & 8 & 28 & 72 & 37.6927 \\ 919 & 11 & 16 & 72 & 37.7825 \\ 921 & 1 & 3 & 73 & 37.8186 \\ 922 & 4 & 2 & 73 & 37.8368 \\ 924 & 7 & 18 & 73 & 37.8420 \\ 925 & 10 & 30 & 73 & 37.8324 \\ 926 & 10 & 28 & 73 & 37.8409 \\ 927 & 9 & 11 & 73 & 37.7412 \\ 928 & 10 & 20 & 73 & 37.7269 \\ 929 & 10 & 14 & 73 & 37.8425 \\ 930 & 9 & 23 & 73 & 37.7467 \\ 938 & 6 & 20 & 74 & 37.8173 \\ 945 & 4 & 8 & 76 & 37.8173 \\ 946 & 7 & 27 & 76 & 37.7797 \\ 947 & 8 & 10 & 76 & 37.7943 \\ 955 & 4 & 24 & 77 & 37.8164 \\ 956 & 5 & 20 & 77 & 37.8552\end{array}$

$\begin{array}{lllll}81.7799 & 424.0 & 427.0 & 1049.4\end{array}$ $82.1701 \quad 330.7 \quad 331.6 \quad 1101.2$ $\begin{array}{lllr}81.8184 & 294.4 & 296.6 & 892.5\end{array}$ 81.8756 81.9794 81.9369 81.8721 82.0049 81.9560 81.8953 81.8464 81.9518 81.9594 81.9454 81.9535 81.9228 81.9656 81.9832 81.9740 81.9875 81.6535 81.6776 81.6755 81.6995 81.7396 B1. 9393
$540.7 \quad 543.21201 .8$

$\begin{array}{lll}188.4 & 189.3 & 482.5\end{array}$

$284.7 \quad 285.3 \quad 605.3$

$440.1 \quad 440.7 \quad 1089.1$

$\begin{array}{llll}235.0 & 238.0 & 795.8\end{array}$

$293.2 \quad 293.8 \quad 907.4$ $\begin{array}{llll}463.6 & 467.0 & 1154.6\end{array}$

634.9

$274.9275 .5 \quad 901.3$

$261.5 \quad 262.1 \quad 868.1$

247.5

269.4

$\begin{array}{lll}257.3 & 260.3 & 905.6\end{array}$

$\begin{array}{lll}327.4 & 329.8 & 793.7\end{array}$

$\begin{array}{lll}510.5 & 512.7 & 959.2\end{array}$

$\begin{array}{lll}333.8 & 336.8 & 779.7\end{array}$

$292.9 \quad 295.0 \quad 752.2$

578.2

$458.7 \quad 460.91079 .9$

605.9608 .11240 .8

$645.3 \quad 647.4 \quad 1281.4$

$\begin{array}{llll}698.0 & 701.0 & 1323.7\end{array}$

$242.0 \quad 244.4674 .8$
$29.4 \quad 12.1 \quad 16.5$ $\begin{array}{lll}41.7 & 12.8 & 26.2\end{array}$ $\begin{array}{lll}35.6 & 13.3 & 25.0\end{array}$ $\begin{array}{lll}36.1 & 11.0 & 20.9\end{array}$ $18.9 \quad 14.2 \quad 9.8$ $\begin{array}{lll}26.7 & 13.3 & 22.1\end{array}$ $34.412 .0 \quad 20.6$ $28.9 \quad 14.0 \quad 18.7$ $47.2 \quad 13.4 \quad 37.3$ $33.311 .9 \quad 18.6$ $\begin{array}{lll}36.7 & 10.1 & 21.7\end{array}$ $31.1 \quad 13.6 \quad 19.4$ $\begin{array}{llll}46.7 & 13.7 \quad 38.0\end{array}$ $\begin{array}{lll}26.7 & 13.8 & 17.7\end{array}$ $\begin{array}{lll}32.8 & 13.6 & 21.4\end{array}$ $\begin{array}{lll}35.0 & 13.7 & 23.5\end{array}$ $\begin{array}{lll}28.3 & 13.2 & 19.1\end{array}$ $26.7 \quad 11.4 \quad 15.9$ $40.6 \quad 13.0 \quad 35.4$ 27.213 .518 .2 $\begin{array}{llll}34.4 & 10.6 & 19.9\end{array}$ $\begin{array}{lll}34.4 & 11.8 & 21.0\end{array}$ $\begin{array}{lll}36.1 & 10.4 & 20.7\end{array}$ $\begin{array}{llll}29.4 & 10.0 & 15.2\end{array}$ $\begin{array}{lll}32.2 & 9.4 & 17.2\end{array}$ $29.413 .8 \quad 23.1$

$\begin{array}{ll}7.7 & 23.8 \\ 8.0 & 33.5 \\ 6.5 & 32.3 \\ 8.8 & 28.2 \\ 3.3 & 16.6 \\ 4.3 & 29.1 \\ 7.9 & 27.9 \\ 5.8 & 25.9 \\ 6.6 & 44.6 \\ 8.4 & 25.9 \\ 8.9 & 29.0 \\ 6.6 & 26.7 \\ 6.3 & 45.2 \\ 5.2 & 24.9 \\ 6.5 & 28.7 \\ 6.6 & 30.8 \\ 5.7 & 26.3 \\ 7.0 & 23.2 \\ 5.6 & 42.6 \\ 5.4 & 25.5 \\ 8.7 & 27.2 \\ 7.9 & 28.3 \\ 9.0 & 28.0 \\ 9.3 & 22.5 \\ 9.6 & 24.5 \\ 4.8 & 30.3\end{array}$


McDowe11 County

\begin{tabular}{|c|c|c|c|c|c|c|c|c|c|c|c|c|c|}
\hline $\begin{array}{l}\text { IFI, } \\
\text { NO }\end{array}$ & & & & $\begin{array}{l}\text { LATITUDE } \\
\text { DEGREES }\end{array}$ & $\begin{array}{l}\text { NG ITUDE } \\
\text { GREES }\end{array}$ & MV & $\begin{array}{c}3 \mathrm{ELV} \\
\mathrm{H}\end{array}$ & $\begin{array}{c}\text { DEPTH } \\
M\end{array}$ & $\underset{\mathrm{C}}{\mathrm{BHT}}$ & $\begin{array}{c}\text { STEMP } \\
\mathrm{C}\end{array}$ & ENT & $\begin{array}{l}\text { HTCOR } \\
\text { C }\end{array}$ & $\begin{array}{c}\text { AAPGRA } \\
\text { C/KM }\end{array}$ \\
\hline $\begin{array}{l}608 \\
609 \\
609 \\
610 \\
611 \\
613 \\
617 \\
618 \\
619 \\
623 \\
624 \\
629 \\
631 \\
639 \\
648 \\
649 \\
654 \\
666 \\
700 \\
727 \\
728 \\
730 \\
742 \\
744 \\
770\end{array}$ & $\begin{array}{r}9 \\
8 \\
10 \\
9 \\
10 \\
10 \\
1 \\
1 \\
11 \\
5 \\
8 \\
9 \\
10 \\
8 \\
1 \\
11 \\
2 \\
8 \\
7 \\
11 \\
10 \\
10 \\
11 \\
3 \\
7 \\
12\end{array}$ & $\begin{array}{r}2 \\
10 \\
10 \\
16 \\
22 \\
24 \\
29 \\
2 \\
17 \\
30 \\
7 \\
14 \\
26 \\
25 \\
5 \\
3 \\
19 \\
22 \\
17 \\
2 \\
9 \\
21 \\
12 \\
14 \\
17 \\
14\end{array}$ & $\begin{array}{l}73 \\
73 \\
73 \\
73 \\
73 \\
73 \\
74 \\
74 \\
73 \\
74 \\
74 \\
74 \\
74 \\
75 \\
76 \\
75 \\
76 \\
76 \\
78 \\
77 \\
77 \\
77 \\
77 \\
78 \\
78\end{array}$ & $\begin{array}{l}37.4808 \\
37.3122 \\
37.4911 \\
37.3628 \\
37.4992 \\
37.3972 \\
37.3372 \\
37.4912 \\
37.3563 \\
37.3434 \\
37.3521 \\
37.3961 \\
37.4959 \\
37.3604 \\
37.3733 \\
37.4047 \\
37.4046 \\
37.3004 \\
37.3810 \\
37.2938 \\
37.3270 \\
37.3162 \\
37.4558 \\
37.3878 \\
37.3100 \\
37.3617\end{array}$ & $\begin{array}{l}81.9270 \\
81.4162 \\
81.9239 \\
81.3496 \\
81.9187 \\
81.3281 \\
81.7628 \\
81.9444 \\
81.7700 \\
81.7535 \\
81.7842 \\
81.8738 \\
81.8861 \\
81.7863 \\
81.4279 \\
81.4616 \\
81.3591 \\
81.7461 \\
81.4080 \\
81.4119 \\
81.4050 \\
81.3965 \\
81.3999 \\
81.4154 \\
81.4256 \\
81.3620\end{array}$ & $\begin{array}{l}443.2 \\
755.0 \\
494.4 \\
794.9 \\
469.1 \\
890.0 \\
535.8 \\
414.5 \\
634.0 \\
373.4 \\
600.2 \\
402.9 \\
422.5 \\
639.5 \\
749.2 \\
556.3 \\
457.8 \\
473.7 \\
751.0 \\
609.9 \\
751.0\end{array}$ & $\begin{array}{l}446.8 \\
758.3 \\
497.7 \\
798.6 \\
472.1 \\
893.4 \\
538.3 \\
417.6 \\
637.0 \\
375.2 \\
603.8 \\
406.0 \\
425.5 \\
642.2 \\
752.2 \\
558.7 \\
460.2 \\
476.4 \\
753.8 \\
612.3 \\
753.5 \\
656.5\end{array}$ & $\begin{array}{l}1246.9 \\
1342.9 \\
1289.9 \\
1448.4 \\
1246.3 \\
1507.5 \\
1387.4 \\
1788.6 \\
1498.4 \\
1218.6 \\
1467.0 \\
1243.0 \\
1198.8 \\
1495.3 \\
1560.3 \\
1194.5 \\
1348.7 \\
1378.3 \\
1356.4 \\
1204.6 \\
1372.5 \\
1268.0 \\
1261.6 \\
1413.4 \\
1359.7\end{array}$ & $\begin{array}{l}35.0 \\
36.7 \\
38.3 \\
38.3 \\
35.0 \\
39.4 \\
35.0 \\
45.6 \\
36.7 \\
37.2 \\
40.6 \\
32.2 \\
37.2 \\
37.8 \\
40.0 \\
33.9 \\
36.7 \\
42.2 \\
33.3 \\
37.8 \\
33.3 \\
35.6 \\
39.4 \\
45.0 \\
33.3 \\
40.6\end{array}$ & $\begin{array}{r}12.4 \\
9.6 \\
11.9 \\
9.1 \\
12.1 \\
8.1 \\
11.7 \\
12.7 \\
10.7 \\
13.3 \\
11.0 \\
12.9 \\
12.6 \\
10.7 \\
9.6 \\
11.4 \\
12.4 \\
12.4 \\
9.5 \\
11.0 \\
9.6 \\
10.6 \\
10.2 \\
13.9 \\
9.6 \\
10.3\end{array}$ & $\begin{array}{l}18.1 \\
20.2 \\
20.5 \\
20.2 \\
18.4 \\
20.8\end{array}$ & $\begin{array}{r}9.1 \\
8.7 \\
10.8 \\
11.2 \\
8.7 \\
9.8 \\
10.0 \\
9.8 \\
8.8 \\
9.9 \\
9.2 \\
9.2 \\
10.2 \\
9.9 \\
9.6\end{array}$ & $\begin{array}{l}25.4 \\
27.4 \\
27.8 \\
27.4 \\
25.6 \\
28.0\end{array}$ \\
\hline
\end{tabular}

Marion County

$\begin{array}{rrrrrrrrrrrrrr}293 & 5 & 12 & 67 & 39.5244 & 80.4451 & 323.4 & 323.4 & 640.1 & 23.9 & 11.2 & 19.9 & 4.6 & 27.0 \\ 294 & 1 & 31 & 68 & 39.5229 & 80.4327 & 338.9 & 339.5 & 962.9 & 30.0 & 11.0 & 19.7 & 7.0 & 27.0 \\ 297 & 8 & 19 & 68 & 39.6329 & 80.4158 & 366.7 & 367.3 & 1142.7 & 36.1 & 10.6 & 22.3 & 8.3 & 29.6 \\ 308 & 9 & 14 & 73 & 39.4749 & 80.3285 & 417.6 & 420.9 & 1585.6 & 40.0 & 10.3 & 18.8 & 11.4 & 25.9 \\ 310 & 10 & 2 & 73 & 39.5203 & 80.2477 & 356.9 & 360.0 & 1558.1 & 38.9 & 10.8 & 18.0 & 11.2 & 25.2 \\ 312 & 2 & 4 & 74 & 39.4370 & 80.0052 & 535.5 & 541.0 & 2409.1 & 60.6 & 9.1 & 21.4 & 15.9 & 28.0 \\ 321 & 7 & 7 & 77 & 39.4236 & 80.0263 & 469.7 & 474.0 & 2359.5 & 58.3 & 9.8 & 20.6 & 15.7 & 27.2\end{array}$

\begin{tabular}{|c|c|c|c|c|c|c|c|c|c|c|c|c|c|}
\hline 69 & & & \multicolumn{11}{|c|}{ Mason County } \\
\hline $\begin{array}{l}6 \\
7\end{array}$ & $\begin{array}{r}8 \\
2 \\
10 \\
11 \\
6 \\
12 \\
12 \\
7 \\
1 \\
2 \\
3 \\
1\end{array}$ & $\begin{array}{r}7 \\
13 \\
27 \\
25 \\
28 \\
20 \\
19 \\
27 \\
23 \\
16 \\
14 \\
8\end{array}$ & $\begin{array}{l}59 \\
61 \\
64 \\
64 \\
65 \\
67 \\
73 \\
75 \\
76 \\
76 \\
78 \\
78\end{array}$ & $\begin{array}{l}38.7138 \\
38.5345 \\
38.7738 \\
38.5929 \\
38.9209 \\
38.5903 \\
38.6970 \\
38.8223 \\
38.9533 \\
38.9341 \\
38.9267 \\
38.9267\end{array}$ & $\begin{array}{l}82.1601 \\
82.0675 \\
82.1785 \\
82.0925 \\
82.1158 \\
82.0703 \\
81.8049 \\
81.8735 \\
81.9802 \\
81.9197 \\
82.0586 \\
82.0586\end{array}$ & $\begin{array}{l}182.0 \\
265.8 \\
192.9 \\
233.8 \\
173.7 \\
233.5 \\
296.6 \\
280.7 \\
232.0 \\
183.8 \\
202.7 \\
202.7\end{array}$ & $\begin{array}{l}185.6 \\
266.4 \\
193.5 \\
235.0 \\
174.0 \\
233.8 \\
298.7 \\
283.8 \\
235.0 \\
186.8 \\
205.7 \\
205.7\end{array}$ & $\begin{array}{r}2631.3 \\
1280.2 \\
552.0 \\
694.0 \\
502.9 \\
706.2 \\
1765.4 \\
1255.2 \\
1546.3 \\
1585.6 \\
1030.2 \\
1037.5\end{array}$ & $\begin{array}{l}53.9 \\
40.0 \\
30.0 \\
28.3 \\
23.9 \\
23.9 \\
44.4 \\
37.2 \\
45.6 \\
40.6 \\
36.7 \\
35.0\end{array}$ & $\begin{array}{l}13.3 \\
12.8 \\
13.2 \\
13.0 \\
13.2 \\
13.0 \\
12.3 \\
12.3 \\
12.6 \\
13.1 \\
12.9 \\
12.9\end{array}$ & $\begin{array}{l}15.4 \\
21.3 \\
30.5 \\
22.1 \\
21.2 \\
15.4 \\
18.2 \\
19.9 \\
21.3 \\
17.3 \\
23.1 \\
21.3\end{array}$ & $\begin{array}{r}16.8 \\
9.3 \\
3.9 \\
5.0 \\
3.5 \\
5.1 \\
12.5 \\
9.1 \\
11.1 \\
11.4 \\
7.5 \\
7.6\end{array}$ & $\begin{array}{l}21.8 \\
28.6 \\
37.5 \\
29.3 \\
28.2 \\
22.6 \\
25.3 \\
27.2 \\
28.5 \\
24.5 \\
30.4 \\
28.6\end{array}$ \\
\hline & & \multicolumn{12}{|c|}{ Mercer County } \\
\hline & $\begin{array}{r}12 \\
11 \\
11 \\
11 \\
4 \\
3 \\
10 \\
4 \\
4 \\
4 \\
10 \\
8 \\
8 \\
9\end{array}$ & $\begin{array}{r}10 \\
20 \\
29 \\
28 \\
7 \\
24 \\
6 \\
6 \\
19 \\
24 \\
6 \\
28 \\
1 \\
23\end{array}$ & $\begin{array}{l}63 \\
64 \\
64 \\
72 \\
73 \\
73 \\
73 \\
74 \\
74 \\
74 \\
74 \\
77 \\
77 \\
77\end{array}$ & $\begin{array}{l}37.5519 \\
37.3542 \\
37.5141 \\
37.4113 \\
37.4824 \\
37.4495 \\
37.3272 \\
37.4408 \\
37.3974 \\
37.3974 \\
37.3689 \\
37.3596 \\
37.4031 \\
37.4713\end{array}$ & $\begin{array}{l}81.1715 \\
81.1888 \\
81.1764 \\
81.3001 \\
81.2158 \\
81.2455 \\
81.3430 \\
81.0183 \\
81.0702 \\
81.0702 \\
81.3329 \\
81.3031 \\
81.2901 \\
81.2697\end{array}$ & $\begin{array}{l}896.4 \\
793.7 \\
791.9 \\
907.4 \\
802.2 \\
801.6 \\
748.0 \\
786.7 \\
776.3 \\
776.3 \\
767.5 \\
731.8 \\
793.4 \\
784.3\end{array}$ & $\begin{array}{l}897.0 \\
797.4 \\
794.3 \\
910.7 \\
805.6 \\
805.0 \\
751.6 \\
789.7 \\
779.4 \\
779.4 \\
770.8 \\
733.7 \\
795.5 \\
787.3\end{array}$ & $\begin{array}{r}1404.5 \\
2749.9 \\
1409.7 \\
1522.8 \\
1351.8 \\
1371.9 \\
1414.0 \\
1351.8 \\
491.0 \\
1427.4 \\
1440.8 \\
1399.9 \\
1432.6 \\
1342.6\end{array}$ & $\begin{array}{l}41.1 \\
58.9 \\
35.0 \\
38.3 \\
40.0 \\
33.9 \\
42.2 \\
34.4 \\
23.9 \\
36.7 \\
38.9 \\
39.4 \\
40.6 \\
44.4\end{array}$ & $\begin{array}{l}7.9 \\
9.1 \\
8.9 \\
7.9 \\
8.9 \\
8.9 \\
9.6 \\
9.1 \\
9.2 \\
9.2 \\
9.4 \\
9.8 \\
9.1 \\
9.1\end{array}$ & $\begin{array}{l}23.7 \\
18.1 \\
18.5 \\
20.0 \\
23.0 \\
18.2 \\
23.1 \\
18.8 \\
29.8 \\
19.2 \\
20.5 \\
21.2 \\
22.0 \\
26.3\end{array}$ & $\begin{array}{r}10.2 \\
17.3 \\
10.2 \\
11.0 \\
9.8 \\
9.9 \\
10.2 \\
9.8 \\
3.4 \\
10.3 \\
10.4 \\
10.1 \\
10.4 \\
9.7\end{array}$ & $\begin{array}{l}30.9 \\
24.4 \\
25.7 \\
27.2 \\
30.3 \\
25.4 \\
30.3 \\
26.0 \\
36.7 \\
26.4 \\
27.7 \\
28.4 \\
29.2 \\
33.6\end{array}$ \\
\hline
\end{tabular}


Mingo County

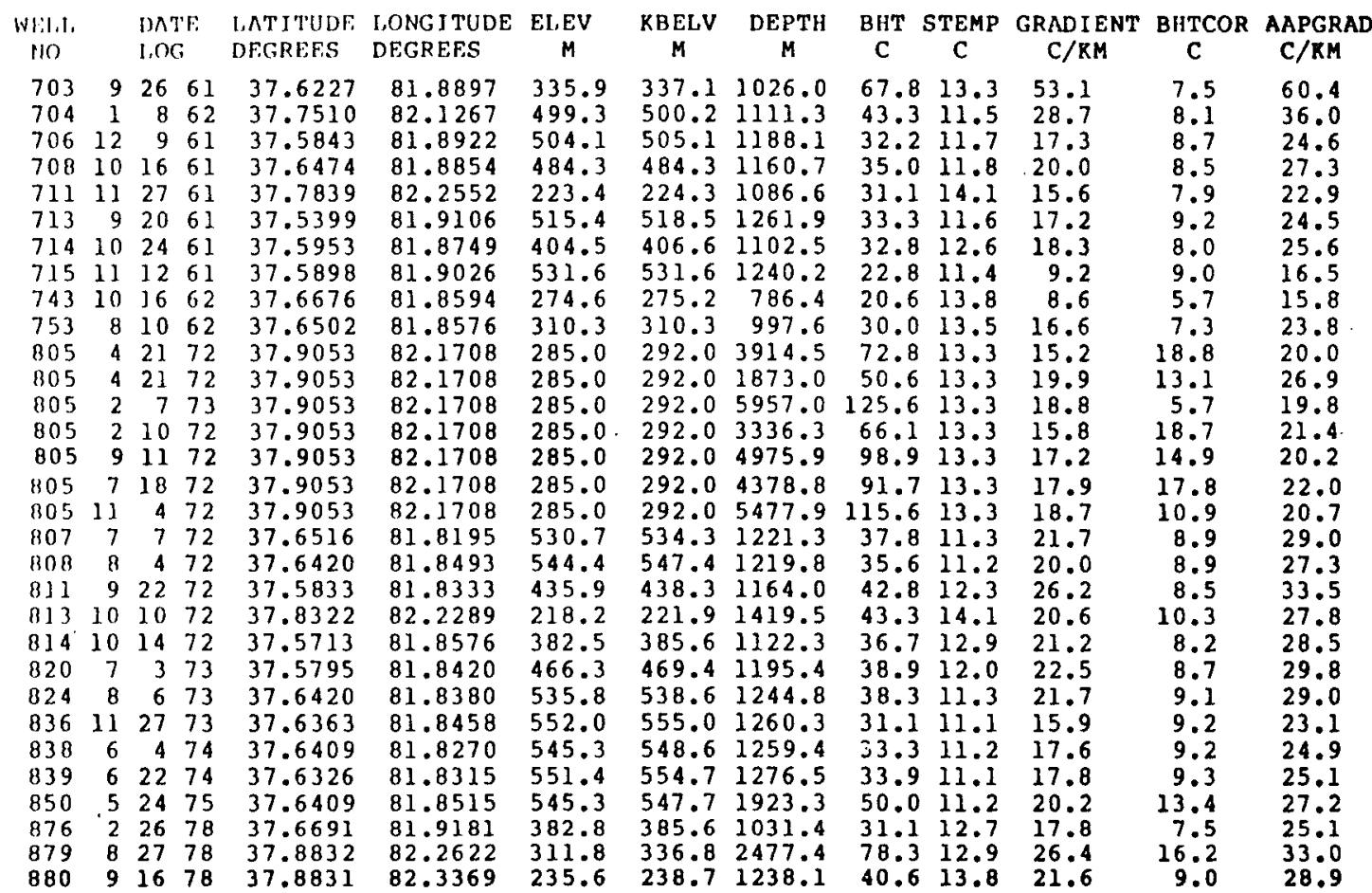

Monroe County

$\begin{array}{rrrrrrrrrrrrrrr}6 & 5 & 11 & 74 & 37.5704 & 80.7526 & 584.9 & 588.6 & 1104.6 & 31.7 & 10.8 & 18.8 & 8.1 & 26.1 \\ 7 & 5 & 26 & 74 & 37.6299 & 80.6802 & 668.4 & 671.5 & 1065.6 & 29.4 & 9.9 & 18.3 & 7.8 & 25.6\end{array}$

Morgan County

$\begin{array}{rrrrrrrrrrrrrr}4 & 4 & 13 & 67 & 39.5849 & 78.4377 & 168.6 & 169.5 & 1418.8 & 36.7 & 12.4 & 17.1 & 10.3 & 24.3 \\ 9 & 11 & 17 & 78 & 39.5775 & 78.4420 & 218.2 & 221.3 & 1530.1 & 38.3 & 11.9 & 17.3 & 11.0 & 24.5\end{array}$

Nichols County

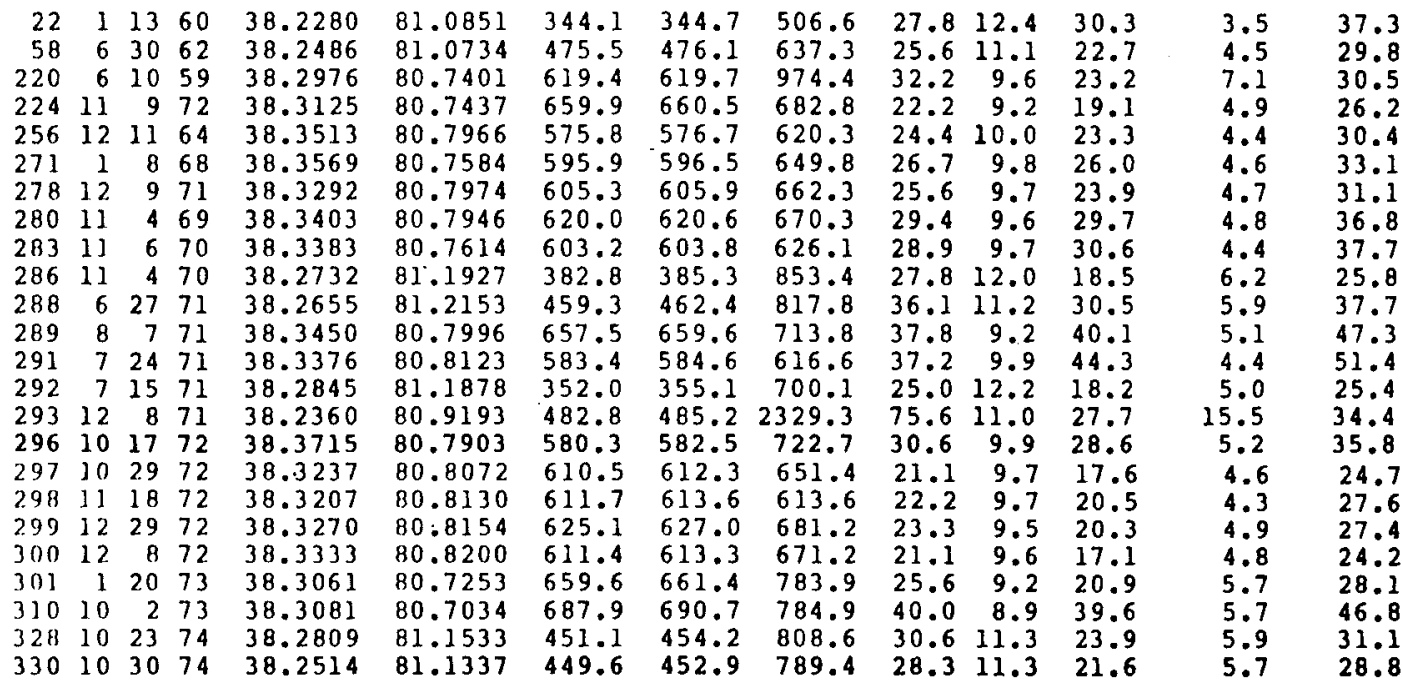


Pendleton County

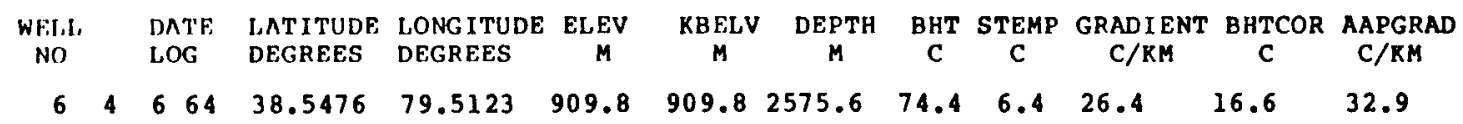

\section{Pleasants County}

$\begin{array}{rrrrrrrrrrrrrr}571 & 12 & 3 & 58 & 39.3673 & 81.1579 & 343.5 & 343.5 & 644.3 & 28.9 & 11.1 & 27.6 & 4.6 & 34.7 \\ 586 & 6 & 16 & 59 & 39.3343 & 81.2699 & 337.7 & 337.7 & 238.7 & 15.6 & 11.2 & 18.2 & 1.4 & 23.9 \\ 626 & 6 & 15 & 64 & 39.3722 & 81.2638 & 268.5 & 269.4 & 376.7 & 23.3 & 11.8 & 30.5 & 2.5 & 37.1 \\ 628 & 8 & 18 & 64 & 39.3741 & 81.2670 & 246.6 & 247.2 & 481.3 & 23.3 & 12.1 & 23.4 & 3.3 & 30.3 \\ 647 & 12 & 20 & 68 & 39.3495 & 81.2717 & 235.3 & 235.9 & 1067.1 & 35.0 & 12.2 & 21.4 & 7.8 & 28.7 \\ 648 & 11 & 1 & 68 & 39.3549 & 81.2165 & 218.5 & 222.2 & 1220.1 & 34.4 & 12.3 & 18.1 & 8.9 & 25.4 \\ 667 & 2 & 17 & 71 & 39.3295 & 81.2880 & 326.7 & 330.7 & 2091.2 & 63.9 & 11.3 & 25.2 & 14.4 & 32.0 \\ 667 & 1 & 12 & 71 & 39.3295 & 81.2880 & 326.7 & 330.7 & 1553.3 & 40.6 & 11.3 & 18.8 & 11.2 & 26.0 \\ 699 & 9 & 5 & 77 & 39.3309 & 81.3546 & 233.2 & 234.7 & 673.3 & 26.7 & 12.2 & 21.5 & 4.8 & 28.6\end{array}$

\section{Pocahontas County}

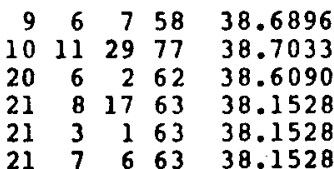

$217663 \quad 38.1528$
$79.75351010 .4 \quad 1010.41774 .5$ $\begin{array}{lllll}79.7476 & 1074.1 & 1074.1 & 1866.6 \\ 79.6466 & 1081.7 & 1085.7 & 1862.6\end{array}$ $80.01151064 .7 \quad 1068.3 \quad 3604.6$ 80.01151064 .71068 .31768 .1 $80.01151064 .71068 .3 \quad 3060.8$ $\begin{array}{lll}61.7 & 5.3 & 31.8\end{array}$ $\begin{array}{lll}48.9 & 4.6 & 23.7\end{array}$ $\begin{array}{lll}55.6 & 4.6 & 27.4\end{array}$ $\begin{array}{lll}77.8 & 5.3 & 20.1\end{array}$ $\begin{array}{lll}40.0 & 5.3 & 19.6 \\ 70.0 & 5.3 & 21.1\end{array}$
12.5

13.1

13.1

$18.9 \quad 25.3$

$12.5 \quad 26.7$

$18.2 \quad 27.1$

\section{Preston County}

\section{Putnam County}

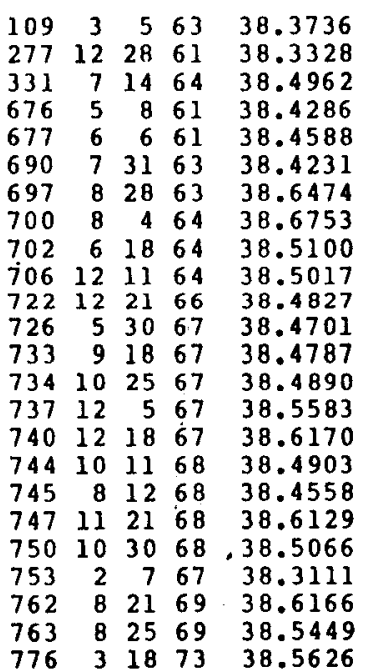

81.9534 81.9482 82.0186 81.8544 82.0123 81.9212 81.8055 81.7518 81.7598 81.7595 81.8507 81.7291 81.7291 81.9026 82.0172 81.7206 81.8568 81.7614 81.8474 81.8807 81.9842 81.7572 81.7697 82.0383
224.0 228.6 191.1 191.1471 .8 $\begin{array}{lll}182.9 & 183.5 & 670.9\end{array}$ $238.0 \quad 238.7 \quad 708.1$ $\begin{array}{llll}295.7 & 295.7 & 1493.5\end{array}$

$289.3 \quad 291.7 \quad 1585.9$ $300.5 \quad 303.01541 .4$ $285.9288 .6 \quad 1515.8$ $287.1 \quad 289.6 \quad 1687.4$

$191.4 \quad 196.9 \quad 1642.6$

204.2210 .01378 .0 $257.6 \quad 260.0 \quad 1417.6$ $209.4 \quad 211.51713 .0$ 324.0327 .11852 .0 $275.8280 .1 \quad 1451.2$ $226.8 \quad 231.3 \quad 1663.6$ $273.1 \quad 276.5 \quad 1962.9$ $252.4 \quad 256.6 \quad 1421.6$ $201.5 \quad 203.91389 .9$ $214.0 \quad 218.5 \quad 1723.9$ $214.3 \quad 218.5 \quad 1700.2$ 203.9207 .31681 .3 $\begin{array}{llll}29.4 & 13.4 & 21.4\end{array}$ $22.213 .4 \quad 13.8$ $\begin{array}{lll}22.2 & 13.5 & 18.4\end{array}$ $\begin{array}{lll}37.2 & 13.4 & 20.2\end{array}$ $\begin{array}{lll}27.2 & 13.7 & 20.2\end{array}$ $\begin{array}{lll}28.9 & 13.2 & 22.2\end{array}$ $\begin{array}{lll}47.8 & 12.4 & 23.7\end{array}$ $\begin{array}{llll}48.3 & 12.4 & 22.7\end{array}$ $\begin{array}{llll}52.2 & 12.5 & 25.8\end{array}$ $\begin{array}{lll}51.7 & 12.6 & 25.8\end{array}$ $45.0 \quad 12.6 \quad 19.2$ $44.4 \quad 13.5 \quad 18.8$ 46.713 .424 .1 $40.6 \quad 12.9 \quad 19.5$ $\begin{array}{llll}43.3 & 13.3 & 17.6\end{array}$ 47.812 .119 .3 $48.912 .7 \quad 24.9$ $57.2 \quad 13.2 \quad 26.5$ $51.1 \quad 12.6 \quad 19.6$ $43.312 .9 \quad 21.4$ $\begin{array}{llll}45.6 & 13.6 & 23.0\end{array}$ $\begin{array}{lll}55.6 & 13.2 & 24.6\end{array}$ $52.8 \quad 13.2 \quad 23.3$ $51.113 .3 \quad 22.5$

$\begin{array}{rr}5.4 & 28.6 \\ 4.6 & 20.9 \\ 3.2 & 25.2 \\ 8.6 & 27.5 \\ 4.8 & 27.4 \\ 5.1 & 29.4 \\ 10.8 & 30.9 \\ 11.4 & 29.8 \\ 11.1 & 33.0 \\ 10.9 & 33.0 \\ 12.0 & 26.3 \\ 11.7 & 26.0 \\ 10.0 & 31.4 \\ 10.3 & 26.8 \\ 12.2 & 24.7 \\ 13.0 & 26.3 \\ 10.5 & 32.2 \\ 11.9 & 33.6 \\ 13.6 & 26.6 \\ 10.3 & 28.6 \\ 10.1 & 30.2 \\ 12.2 & 31.7 \\ 12.1 & 30.4 \\ 12.0 & 29.6\end{array}$


Raleigh County

\begin{tabular}{|c|c|c|c|c|c|c|c|c|c|c|c|c|c|}
\hline \multicolumn{2}{|c|}{$\begin{array}{c}\text { WELI, } \\
\text { NO }\end{array}$} & \multicolumn{2}{|c|}{$\begin{array}{l}\text { DATE } \\
\text { LOG }\end{array}$} & $\begin{array}{l}\text { JATITUDE } \\
\text { DEGREES }\end{array}$ & $\begin{array}{l}\text { LONGITUDE } \\
\text { DEGREES }\end{array}$ & $\begin{array}{c}\text { ELEV } \\
M\end{array}$ & $\underset{\mathbf{M}}{\text { KBELV }}$ & $\begin{array}{c}\text { DEPTH } \\
\mathbf{M}\end{array}$ & $\begin{array}{c}\mathrm{BHT} \\
\mathrm{C}\end{array}$ & $\begin{array}{c}\text { STEMP } \\
\mathrm{C}\end{array}$ & $\begin{array}{c}\text { GRADI ENT } \\
\text { C/KM }\end{array}$ & $\underset{\mathrm{C}}{\mathrm{BHTCOR}}$ & $\begin{array}{c}\text { AAPGRAD } \\
\text { C/XM }\end{array}$ \\
\hline $\begin{array}{l}262 \\
266 \\
267 \\
269 \\
274 \\
277 \\
281 \\
288 \\
289 \\
290 \\
291 \\
296 \\
307 \\
308 \\
319 \\
320\end{array}$ & $\begin{array}{r}3 \\
12 \\
4 \\
7 \\
4 \\
3 \\
1 \\
12 \\
11 \\
10 \\
1 \\
2 \\
9 \\
10 \\
7 \\
8\end{array}$ & $\begin{array}{r}21 \\
18 \\
27 \\
13 \\
7 \\
5 \\
12 \\
6 \\
19 \\
27 \\
20 \\
13 \\
17 \\
3 \\
25 \\
16\end{array}$ & $\begin{array}{l}63 \\
63 \\
64 \\
64 \\
65 \\
65 \\
65 \\
70 \\
71 \\
71 \\
72 \\
73 \\
73 \\
73 \\
74 \\
74\end{array}$ & $\begin{array}{l}37.6529 \\
37.5762 \\
37.5542 \\
37.7080 \\
37.5820 \\
37.5652 \\
37.7921 \\
37.5583 \\
37.8300 \\
37.8374 \\
37.7495 \\
37.7322 \\
37.5515 \\
37.5438 \\
37.5421 \\
37.5490\end{array}$ & $\begin{array}{l}81.3335 \\
81.2048 \\
81.2055 \\
81.2847 \\
81.3020 \\
81.2555 \\
81.3472 \\
81.2065 \\
81.3115 \\
81.3351 \\
81.3781 \\
80.9828 \\
81.2594 \\
81.2576 \\
81.2690 \\
81.2812\end{array}$ & $\begin{array}{l}590.7 \\
991.5 \\
807.4 \\
755.0 \\
802.8 \\
890.6 \\
616.9 \\
869.0 \\
548.0 \\
541.9 \\
623.9 \\
857.1 \\
682.1 \\
795.8 \\
793.7 \\
743.1\end{array}$ & $\begin{array}{l}591.3 \\
991.5 \\
807.4 \\
755.6 \\
805.3 \\
893.7 \\
620.6 \\
872.0 \\
551.1 \\
545.0 \\
627.0 \\
862.0 \\
685.2 \\
798.9 \\
796.7 \\
746.2\end{array}$ & $\begin{array}{r}1014.4 \\
1519.7 \\
777.2 \\
620.6 \\
1252.1 \\
1372.5 \\
1008.6 \\
1180.2 \\
991.8 \\
1032.7 \\
1068.9 \\
2672.8 \\
1127.8 \\
1197.9 \\
1234.4 \\
1207.3\end{array}$ & $\begin{array}{l}35.0 \\
44.4 \\
24.4 \\
30.0 \\
35.6 \\
33.3 \\
28.9 \\
41.1 \\
31.1 \\
37.2 \\
32.8 \\
69.4 \\
27.2 \\
34.4 \\
36.7 \\
35.0\end{array}$ & $\begin{array}{r}10.7 \\
6.9 \\
8.8 \\
9.1 \\
8.7 \\
7.9 \\
10.3 \\
8.1 \\
10.9 \\
10.9 \\
10.3 \\
8.0 \\
10.0 \\
8.9 \\
8.9 \\
9.4\end{array}$ & $\begin{array}{l}23.9 \\
24.7 \\
20.2 \\
33.8 \\
21.4 \\
18.5 \\
18.5 \\
28.0 \\
20.4 \\
25.4 \\
21.1 \\
23.0 \\
15.3 \\
21.4 \\
22.5 \\
21.2\end{array}$ & $\begin{array}{r}7.4 \\
20.9 \\
5.6 \\
4.4 \\
9.1 \\
9.9 \\
7.4 \\
8.6 \\
7.2 \\
7.5 \\
7.8 \\
17.0 \\
8.2 \\
8.7 \\
9.0 \\
8.8\end{array}$ & $\begin{array}{l}31.2 \\
31.9 \\
27.4 \\
40.8 \\
28.7 \\
25.8 \\
25.8 \\
35.3 \\
27.7 \\
32.7 \\
28.4 \\
29.4 \\
22.6 \\
28.7 \\
29.8 \\
28.5\end{array}$ \\
\hline
\end{tabular}

\section{Randolph County}

$\begin{array}{rrrrr}175 & 6 & 25 & 74 & 38.8753 \\ 176 & 7 & 15 & 74 & 38.8145 \\ 178 & 7 & 20 & 76 & 38.8232 \\ 186 & 9 & 4 & 77 & 38.7850 \\ 188 & 7 & 24 & 77 & 38.8560 \\ 189 & 7 & 30 & 77 & 38.8638 \\ 190 & 8 & 7 & 77 & 38.8675 \\ 194 & 8 & 13 & 77 & 38.8683 \\ 217 & 5 & 19 & 78 & 38.8009 \\ 222 & 6 & 16 & 78 & 38.9098 \\ 224 & 6 & 4 & 78 & 38.8149 \\ 227 & 5 & 25 & 78 & 38.8204 \\ 228 & 5 & 5 & 78 & 38.7952 \\ 230 & 6 & 30 & 78 & 38.8604 \\ 232 & 7 & 24 & 78 & 38.8583\end{array}$

80.0291 80.0388 80.0624 80.0995 80.0762 80.0751 80.0829 80.0904 80.0960 80.0081 80.0671 80.0539 80.0919 80.0705 80.0890

$\begin{array}{rlllllll}826.9 & 830.6 & 1461.8 & 44.4 & 6.9 & 25.7 & 10.6 & 32.9 \\ 675.7 & 679.7 & 1143.0 & 38.3 & 8.4 & 26.2 & 8.3 & 33.5 \\ 819.9 & 823.0 & 2412.8 & 78.9 & 7.0 & 29.8 & 15.9 & 36.4 \\ 896.4 & 898.9 & 1613.6 & 50.0 & 6.3 & 27.1 & 11.5 & 34.2 \\ 801.3 & 803.8 & 1582.2 & 40.6 & 7.1 & 21.1 & 11.3 & 28.3 \\ 746.8 & 749.2 & 1555.4 & 45.0 & 7.7 & 24.0 & 11.2 & 31.2 \\ 576.1 & 578.5 & 1401.5 & 50.0 & 9.3 & 29.0 & 10.1 & 36.3 \\ 692.5 & 694.9 & 1477.4 & 51.1 & 8.2 & 29.1 & 10.7 & 36.3 \\ 922.9 & 926.0 & 1622.5 & 42.8 & 6.0 & 22.7 & 11.6 & 29.8 \\ 738.8 & 741.9 & 1556.3 & 41.1 & 7.7 & 21.5 & 11.2 & 28.7 \\ 798.9 & 801.3 & 1505.1 & 42.2 & 7.2 & 23.3 & 10.8 & 30.5 \\ 713.8 & 716.6 & 1433.5 & 56.1 & 8.0 & 33.5 & 10.4 & 40.8 \\ 843.7 & 846.7 & 1557.5 & 50.0 & 6.8 & 27.7 & 11.2 & 34.9 \\ 592.2 & 595.3 & 1402.1 & 55.0 & 9.2 & 32.7 & 10.2 & 39.9 \\ 708.4 & 711.4 & 1536.8 & 43.3 & 8.0 & 23.0 & 11.0 & 30.2\end{array}$

Ritchie County

$\begin{array}{rrrrr}3019 & 4 & 6 & 66 & 39.1366 \\ 3133 & 7 & 14 & 66 & 39.1823 \\ 3139 & 11 & 14 & 66 & 39.1755 \\ 3218 & 8 & 28 & 67 & 39.0942 \\ 3330 & 10 & 11 & 69 & 39.2596 \\ 3337 & 12 & 3 & 69 & 39.2357 \\ 3358 & 3 & 31 & 70 & 39.2459 \\ 3364 & 5 & 22 & 70 & 39.2376 \\ 3365 & 6 & 10 & 70 & 39.2168 \\ 3368 & 5 & 8 & 70 & 39.0378 \\ 3380 & 8 & 11 & 70 & 39.0202 \\ 3384 & 10 & 7 & 70 & 39.2338 \\ 3385 & 1 & 22 & 71 & 39.2173 \\ 3402 & 3 & 8 & 71 & 39.2337 \\ 3403 & 4 & 24 & 71 & 39.2728\end{array}$

80.9984
81.1586
80.8723
80.9561
80.9527
81.0806
81.0486
81.0441
80.9960
81.0703
81.0770
81.0822
80.9886
81.0507
81.0674

$\begin{array}{llllll}282.9 & 282.9 & 575.5 & 22.8 & 12.0 & 18.8 \\ 278.0 & 278.6 & 620.3 & 26.7 & 12.0 & 23.7 \\ 321.3 & 321.9 & 650.4 & 27.2 & 11.5 & 24.1 \\ 271.9 & 272.5 & 589.2 & 27.8 & 12.1 & 26.6 \\ 285.0 & 285.0 & 611.1 & 26.7 & 11.8 & 24.3 \\ 266.7 & 266.7 & 632.2 & 20.6 & 12.0 & 13.5 \\ 291.7 & 292.3 & 596.5 & 17.2 & 11.8 & 9.2 \\ 291.4 & 291.4 & 636.7 & 25.0 & 11.8 & 20.8 \\ 318.5 & 319.4 & 635.8 & 26.1 & 11.5 & 22.9 \\ 224.6 & 226.5 & 594.7 & 27.8 & 12.6 & 25.5 \\ 335.3 & 336.5 & 688.5 & 28.9 & 11.6 & 25.2 \\ 248.4 & 249.0 & 559.9 & 18.9 & 12.2 & 12.0 \\ 280.4 & 281.0 & 586.7 & 21.7 & 11.9 & 16.6 \\ 286.5 & 287.1 & 609.9 & 23.3 & 11.8 & 18.9 \\ 310.9 & 310.9 & 639.5 & 21.7 & 11.5 & 15.8\end{array}$

$\begin{array}{ll}4.0 & 25.8 \\ 4.4 & 30.8 \\ 4.6 & 31.2 \\ 4.2 & 33.6 \\ 4.3 & 31.4 \\ 4.5 & 20.6 \\ 4.2 & 16.2 \\ 4.5 & 27.9 \\ 4.5 & 30.0 \\ 4.2 & 32.5 \\ 4.9 & 32.3 \\ 3.9 & 19.0 \\ 4.1 & 23.7 \\ 4.3 & 25.9 \\ 4.5 & 22.9\end{array}$


Roane County

\begin{tabular}{|c|c|c|c|c|c|c|c|c|c|c|c|c|c|}
\hline $\begin{array}{c}\text { WHIIL } \\
\text { NO }\end{array}$ & & $\begin{array}{l}\text { DA: } \\
\text { LOC }\end{array}$ & & $\begin{array}{l}\text { LATITUDE } \\
\text { DEGREES }\end{array}$ & $\begin{array}{l}\text { LONG ITUDE } \\
\text { DEGREES }\end{array}$ & $\begin{array}{c}\text { ELEV } \\
M\end{array}$ & $\begin{array}{c}\text { RBELV } \\
\text { M }\end{array}$ & $\begin{array}{c}\text { DEPTH } \\
M\end{array}$ & $\begin{array}{c}\text { BHT } \\
\text { C }\end{array}$ & $\underset{C}{\text { STEMP }}$ & $\begin{array}{c}\text { GRADI ENT } \\
\text { C/KM }\end{array}$ & $\begin{array}{c}\text { BHTCOR } \\
\text { C }\end{array}$ & $\begin{array}{c}\text { AAPGRAD } \\
\text { C/KM }\end{array}$ \\
\hline $\begin{array}{l}1,645 \\
1663 \\
1676 \\
1676 \\
1682 \\
1723 \\
1724 \\
1745 \\
1942 \\
1983 \\
1984 \\
1986 \\
2002\end{array}$ & $\begin{array}{r}10 \\
1 \\
9 \\
9 \\
11 \\
10 \\
12 \\
9 \\
10 \\
7 \\
6 \\
7 \\
10\end{array}$ & $\begin{array}{r}29 \\
27 \\
12 \\
5 \\
27 \\
27 \\
2 \\
10 \\
17 \\
20 \\
26 \\
2 \\
22\end{array}$ & $\begin{array}{l}68 \\
68 \\
68 \\
68 \\
68 \\
69 \\
69 \\
69 \\
71 \\
73 \\
73 \\
73 \\
73\end{array}$ & $\begin{array}{l}38.6256 \\
38.6167 \\
38.7747 \\
38.7747 \\
38.7878 \\
38.6624 \\
38.6632 \\
38.6431 \\
38.8763 \\
38.8788 \\
38.8741 \\
38.8752 \\
38.8666\end{array}$ & $\begin{array}{l}81.4342 \\
81.3597 \\
81.2153 \\
81.2153 \\
81.5176 \\
81.3583 \\
81.3544 \\
81.4360 \\
81.2883 \\
81.2953 \\
81.2671 \\
81.2583 \\
81.2796\end{array}$ & $\begin{array}{l}284.7 \\
286.5 \\
257.9 \\
257.9 \\
237.7 \\
315.2 \\
324.6 \\
280.7 \\
271.9 \\
323.1 \\
309.4 \\
283.5 \\
323.1\end{array}$ & $\begin{array}{l}285.6 \\
287.1 \\
260.0 \\
260.0 \\
238.7 \\
315.8 \\
325.5 \\
282.9 \\
273.1 \\
325.2 \\
311.5 \\
285.3 \\
326.7\end{array}$ & $\begin{array}{r}634.0 \\
602.0 \\
2424.7 \\
2040.0 \\
477.6 \\
691.3 \\
679.7 \\
661.7 \\
747.1 \\
844.6 \\
670.6 \\
672.1 \\
1786.1\end{array}$ & $\begin{array}{l}26.7 \\
22.2 \\
65.6 \\
60.0 \\
21.7 \\
30.0 \\
28.9 \\
29.4 \\
37.8 \\
42.2 \\
28.3 \\
30.6 \\
53.3\end{array}$ & $\begin{array}{l}12.5 \\
12.5 \\
12.6 \\
12.6 \\
12.8 \\
12.2 \\
12.1 \\
12.5 \\
12.3 \\
2 \\
111.8 \\
3 \\
12.0 \\
5 \\
12.2 \\
111.8\end{array}$ & $\begin{array}{l}22.3 \\
16.2 \\
21.8 \\
23.2 \\
18.6 \\
25.8 \\
24.7 \\
25.6 \\
34.0 \\
36.0 \\
24.4 \\
27.3 \\
23.2\end{array}$ & $\begin{array}{r}4.5 \\
4.3 \\
16.0 \\
14.1 \\
3.3 \\
5.0 \\
4.9 \\
4.7 \\
5.4 \\
6.1 \\
4.8 \\
4.8 \\
12.6\end{array}$ & $\begin{array}{l}29.4 \\
23.2 \\
28.4 \\
30.1 \\
25.5 \\
33.0 \\
31.9 \\
32.7 \\
41.3 \\
43.2 \\
31.5 \\
34.4 \\
30.3\end{array}$ \\
\hline
\end{tabular}

Summers County

$\begin{array}{rrrrr}5 & 10 & 3 & 64 & 37.6921 \\ 6 & 11 & 23 & 64 & 37.6678\end{array}$

80.9253

80.7064

80.7438
$552.9 \quad 555.3 \quad 2519.2$

$471.8 \quad 474.9 \quad 1965.0$

$\begin{array}{rrr}48.3 & 11.8 & 18.6 \\ 36.1 & 9.2 & 28.4\end{array}$
$16.4 \quad 25.3$

$\begin{array}{rr}13.7 & 25.5 \\ 6.9 & 35.7\end{array}$

\section{Taylor County}

$\begin{array}{lllll}95 & 10 & 26 & 71 & 39.3108\end{array}$

$\begin{array}{lllll}96 & 11 & 3 & 71 & 39.3125\end{array}$

$\begin{array}{lllll}104 & 9 & 9 & 74 & 39.3105\end{array}$

$\begin{array}{llllll}167 & 10 & 30 & 78 & 39.2990\end{array}$

$\begin{array}{lllll}170 & 12 & 6 & 78 & 39.3019\end{array}$
79.9252 79.9354 80.0708 79.9394 79.9261
485. $\begin{array}{lll}454.8 & 457.5 & 1286.6\end{array}$ $381.0 \quad 384.7 \quad 1393.2$ $412.4 \quad 415.4 \quad 1232.0$ $472.4 \quad 475.51288 .4$ $\begin{array}{lll}38.9 & 9.7 & 22.7\end{array}$ $\begin{array}{lll}38.9 & 10.0 & 22.4\end{array}$ $40.6 \quad 10.8 \quad 21.4$ $\begin{array}{lll}37.8 & 10.5 & 22.2\end{array}$ $33.99 .9 \quad 18.6$

$\begin{array}{rr}9.4 & 29.9 \\ 9.4 & 29.7 \\ 10.1 & 28.6 \\ 9.0 & 29.4 \\ 9.4 & 25.9\end{array}$

\section{Tucker County}

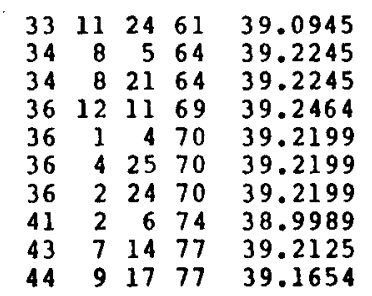

79.4021 79.5836 79.5836 79.7701 79.7675 79.7675 79.7675 79.4776
79.7665 79.7668

$\begin{array}{lll}72.8 & 5.1 & 25.7 \\ 47.2 & 8.5 & 27.5 \\ 62.2 & 8.5 & 25.2 \\ 22.2 & 9.1 & 39.3 \\ 43.9 & 9.1 & 23.8 \\ 76.1 & 9.1 & 25.3 \\ 59.4 & 9.1 & 22.5 \\ 66.1 & 3.8 & 23.3 \\ 48.9 & 7.5 & 24.2 \\ 65.6 & 7.9 & 30.4\end{array}$

$\begin{array}{rr}16.8 & 32.1 \\ 10.2 & 34.8 \\ 14.6 & 32.1 \\ 2.1 & 45.7 \\ 10.6 & 31.0 \\ 16.9 & 31.6 \\ 15.1 & 29.2 \\ 17.0 & 29.7 \\ 12.1 & 31.4 \\ 13.3 & 37.4\end{array}$

\section{Tyler County}

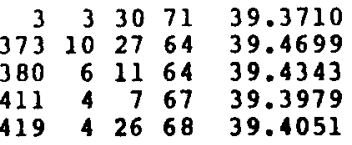
$\begin{array}{rrrr}80.8789 & 295.4 & 296.3 & 670.6 \\ 80.7112 & 346.3 & 346.9 & 927.5\end{array}$ $\begin{array}{llll}80.6858 & 0.314 .6 & 315.2 & 1609.3\end{array}$ $\begin{array}{llll}80.8735 & 350.5 & 351.1 & 939.1\end{array}$ 80.8575250 .5250 .9 . 619.4
$25.0 \quad 11.6$ 48.911 .0

35.611 .0 $\begin{array}{lll}35.6 & 11.0 & 26.1 \\ 20.0 & 12.0 & 12.9\end{array}$
20.0 24.1 23.3

$\begin{array}{rr}4.8 & 27.1 \\ 6.8 & 31.4 \\ 11.5 & 30.5 \\ 6.8 & 33.4 \\ 4.4 & 20.0\end{array}$

\section{Upshur County}

$\begin{array}{rrrrr}1730 & 6 & 24 & 77 & 39.0146 \\ 1741 & 4 & 26 & 77 & 38.9989 \\ 1772 & 9 & 9 & 77 & 38.9024 \\ 1773 & 12 & 20 & 77 & 38.8747 \\ 1794 & 2 & 19 & 78 & 38.9912 \\ 1795 & 3 & 5 & 78 & 38.9846 \\ 1796 & 2 & 26 & 78 & 38.9876 \\ 1799 & 3 & 11 & 78 & 38.9489 \\ 1845 & 9 & 16 & 78 & 38.8996 \\ 1853 & 9 & 18 & 78 & 38.8486 \\ 1876 & 12 & 19 & 78 & 38.8976 \\ 1877 & 12 & 13 & 78 & 38.9922 \\ 1901 & 2 & 26 & 79 & 38.9125 \\ 1902 & 2 & 20 & 79 & 38.9065 \\ 1917 & 3 & 19 & 79 & 38.9967\end{array}$

80.0922 80.1317 80.1295 80.3454 80.2913 80.2947 80.2949 80.1148 80.1255 80.2026 80.2076 80.0886 80.1197 80.1343 80.1554 $\begin{array}{lll}599.8 & 602.9 & 1260.3\end{array}$ 504.1 656.8 466.0 463.9 466.6 463.3 646.5 636.7 567.5 548.6 491.0 627.0

633.1

494.7

\subsection{4 .1}

659.91174 .1 469.11315 .2 $466.6 \quad 1409.4$ 469.71406 .0 649.51196 .9 639.81165 .9 570.61219 .8 551.71250 .6 494.11231 .4 629.71167 .1 636.7
497.7
4288.7 $466.6 \quad 1424.6$ $\begin{array}{lll}39.4 & 8.9 & 24.2\end{array}$ $33.9 \quad 9.9 \quad 19.6$ $\begin{array}{lll}41.7 & 8.5 & 28.2\end{array}$ $\begin{array}{llll}35.6 & 10.4 & 19.1\end{array}$ $41.7 \quad 10.3 \quad 22.2$ $\begin{array}{lll}41.1 & 10.3 & 21.9\end{array}$ $\begin{array}{lll}40.6 & 10.3 & 21.2\end{array}$ $\begin{array}{lll}37.2 & 8.6 & 24.0\end{array}$ $\begin{array}{lll}33.9 & 8.7 & 21.6\end{array}$ $\begin{array}{lll}37.8 & 9.4 & 23.2\end{array}$ $\begin{array}{lll}35.0 & 9.6 & 20.3\end{array}$ $\begin{array}{lll}30.6 & 10.0 & 16.7\end{array}$ $\begin{array}{lll}37.8 & 8.8 & 24.8\end{array}$ $\begin{array}{lll}35.6 & 8.7 & 22.7\end{array}$ $38.9 \quad 10.0 \quad 22.4$

$\begin{array}{rr}9.2 & 31.5 \\ 8.9 & 26.9 \\ 8.6 & 35.5 \\ 9.6 & 26.4 \\ 10.2 & 29.5 \\ 10.2 & 29.2 \\ 10.3 & 28.5 \\ 8.7 & 31.2 \\ 8.5 & 28.9 \\ 8.9 & 30.5 \\ 9.1 & 27.6 \\ 9.0 & 24.0 \\ 8.5 & 32.1 \\ 8.6 & 30.0 \\ 9.4 & 29.7\end{array}$


Wayne County

\begin{tabular}{|c|c|c|c|c|c|c|c|c|c|c|c|c|c|}
\hline $\begin{array}{c}\text { WELLI, } \\
\text { NO }\end{array}$ & & & $\Lambda \mathrm{TE}$ & $\begin{array}{l}\text { LAT ITUDE } \\
\text { DEGREFS }\end{array}$ & $\begin{array}{l}\text { LONGITUDE } \\
\text { DEGREES }\end{array}$ & $\begin{array}{c}E \text { ELEV } \\
M\end{array}$ & $\underset{M}{\text { RBELV }}$ & $\begin{array}{c}\text { DEPTH } \\
M\end{array}$ & $\underset{\mathrm{C}}{\mathrm{BHT}}$ & $\underset{C}{\text { STEMP }}$ & $\begin{array}{c}\text { GRADIENT } \\
\mathrm{C} / \mathrm{KM}\end{array}$ & $\begin{array}{c}\text { IHTCOR } \\
\text { C }\end{array}$ & $\begin{array}{c}\text { AAPGRA } \\
\text { C/RM }\end{array}$ \\
\hline $\begin{array}{l}1555 \\
1572 \\
1572 \\
1572 \\
1572 \\
1572 \\
1572 \\
1572 \\
1572 \\
1572 \\
1572 \\
1572 \\
1572 \\
1572 \\
1572 \\
1572 \\
1576 \\
1581 \\
1603 \\
1604 \\
1607\end{array}$ & $\begin{array}{r}8 \\
10 \\
8 \\
11 \\
7 \\
11 \\
9 \\
10 \\
8 \\
11 \\
9 \\
9 \\
8 \\
9 \\
9 \\
10 \\
10 \\
10 \\
10 \\
10 \\
10\end{array}$ & $\begin{array}{r}27 \\
16 \\
9 \\
9 \\
23 \\
8 \\
11 \\
31 \\
8 \\
9 \\
10 \\
26 \\
10 \\
18 \\
10 \\
16 \\
1 \\
16 \\
11 \\
17 \\
1\end{array}$ & $\begin{array}{l}70 \\
74 \\
74 \\
74 \\
74 \\
74 \\
74 \\
74 \\
74 \\
74 \\
74 \\
74 \\
74 \\
74 \\
74 \\
74 \\
75 \\
75 \\
77 \\
77 \\
78\end{array}$ & $\begin{array}{r}38.2667 \\
38.2219 \\
38.2219 \\
38.2219 \\
38.2219 \\
38.2219 \\
38.2219 \\
38.2219 \\
38.2219 \\
38.2219 \\
38.2219 \\
38.2219 \\
38.2219 \\
38.2219 \\
38.2219 \\
38.2219 \\
38.1230 \\
37.8911 \\
38.0394 \\
38.0405 \\
38.0000\end{array}$ & $\begin{array}{l}82.3027 \\
82.5343 \\
82.5343 \\
82.5343 \\
82.5343 \\
82.5343 \\
82.5343 \\
82.5343 \\
82.5343 \\
82.5343 \\
82.5343 \\
82.5343 \\
82.5343 \\
82.5343 \\
82.5343 \\
82.5343 \\
82.5410 \\
82.3947 \\
82.5021 \\
82.4895 \\
82.3333\end{array}$ & $\begin{array}{l}280.7 \\
181.1 \\
181.1 \\
181.1 \\
181.1 \\
181.1 \\
181.1 \\
181.1 \\
181.1 \\
181.1 \\
181.1 \\
181.1 \\
181.1 \\
181.1 \\
181.1 \\
181.1 \\
194.2 \\
230.4 \\
332.5 \\
250.2 \\
224.0\end{array}$ & $\begin{array}{l}281.0 \\
189.6 \\
189.6 \\
189.6 \\
189.6 \\
189.6 \\
189.6 \\
189.6 \\
189.6 \\
189.6 \\
189.6 \\
189.6 \\
189.6 \\
189.6\end{array}$ & $\begin{array}{r}990.9 \\
4014.2 \\
2217.4 \\
3962.4 \\
590.1 \\
4447.6 \\
3188.5 \\
4331.8 \\
2117.8 \\
4447.6 \\
3188.5 \\
3493.3 \\
2216.8 \\
3371.4 \\
3188.5 \\
4017.0 \\
1095.5 \\
1220.1 \\
536.1 \\
460.2 \\
1204.0\end{array}$ & $\begin{array}{l}35.7 \\
72.8 \\
57.8 \\
81.1 \\
28.3 \\
76.7 \\
60.0 \\
77.2 \\
52.2 \\
76.7 \\
60.0 \\
66.7 \\
58.9 \\
61.1 \\
58.9 \\
76.1 \\
28.9 \\
36.1 \\
25.0 \\
23.9 \\
43.3\end{array}$ & $\begin{array}{l}13.0 \\
13.8 \\
13.8 \\
13.8 \\
13.8 \\
13.8 \\
13.8 \\
13.8 \\
13.8 \\
13.8 \\
13.8 \\
13.8\end{array}$ & $\begin{array}{l}23.8 \\
14.7 \\
19.8 \\
17.0 \\
24.6 \\
14.1 \\
14.5 \\
14.6 \\
18.1 \\
14.1 \\
14.5 \\
15.1 \\
20.3 \\
14.0 \\
14.1 \\
15.5 \\
13.7 \\
18.2\end{array}$ & $\begin{array}{r}7.2 \\
18.7 \\
15.0 \\
18.7 \\
4.2 \\
17.6 \\
18.4 \\
17.9 \\
14.5 \\
17.6 \\
18.4 \\
18.8 \\
15.0 \\
18.7 \\
18.4 \\
18.7 \\
8.0 \\
8.9 \\
3.7 \\
3.1 \\
8.8\end{array}$ & $\begin{array}{l}31.1 \\
19.3 \\
26.6 \\
21.7 \\
31.6 \\
18.1 \\
20.3 \\
18.8\end{array}$ \\
\hline $\begin{array}{l}1609 \\
1610 \\
1615\end{array}$ & $\begin{array}{l}6 \\
6 \\
7\end{array}$ & $\begin{array}{r}6 \\
12 \\
21\end{array}$ & $\begin{array}{l}78 \\
78 \\
78\end{array}$ & $\begin{array}{l}38.0413 \\
38.0474 \\
38.0336\end{array}$ & $\begin{array}{l}82.4734 \\
82.4811 \\
82.4903\end{array}$ & $\begin{array}{l}233.2 \\
244.8 \\
319.1\end{array}$ & $\begin{array}{l}235.3 \\
247.2 \\
321.6\end{array}$ & $\begin{array}{l}443.5 \\
449.3 \\
528.8\end{array}$ & $\begin{array}{l}21.1 \\
30.0 \\
30.0\end{array}$ & $\begin{array}{l}13.6 \\
13.5 \\
12.8\end{array}$ & $\begin{array}{l}16.9 \\
36.7 \\
32.5\end{array}$ & $\begin{array}{l}3.0 \\
3.0 \\
3.7\end{array}$ & $\begin{array}{l}23.6 \\
43.5 \\
39.5\end{array}$ \\
\hline
\end{tabular}

\section{Webster County}

$\begin{array}{llllll}50 & 3 & 23 & 57 & 38 & 4493\end{array}$

$\begin{array}{lllll}55 & 3 & 30 & 68 & 38.5035\end{array}$

80.2587

80.3626

$\begin{array}{llllll}539.8 & 540.7 & 952.2 & 37.2 & 10.2 & 28.4\end{array}$

$6.9 \quad 35.7$

$\begin{array}{llllll}61 & 9 & 14 & 74 & 38.6111\end{array}$

80.5253

$\begin{array}{llllll}.3 & 500.2 & 2651.5 & 66.1 & 10.5 & 21.0\end{array}$

$16.9 \quad 27.3$

\section{Wetzel County}

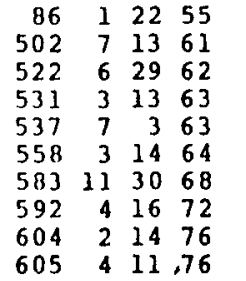

39.6446

39.4771

39.5877

39.5771

39.5955

39.5113

39.4885

39.5305

39.5363

39.4406
80.7600 80.6436 80.5570 80.5542 80.6757 80.5178 80.6609 80.6838 80.5837 80.5526

$\begin{array}{rrr}354.2 & 354.8 & 671.8 \\ 291.1 & 291.7 & 880.6 \\ 242.9 & 243.5 & 960.4 \\ 339.9 & 340.5 & 884.2 \\ 399.3 & 399.9 & 976.6 \\ 365.2 & 365.5 & 967.4 \\ 357.8 & 358.4 & 754.4 \\ 333.5 & 334.7 & 704.7 \\ 289.3 & 290.8 & 976.0\end{array}$

417.0 $\begin{array}{lll}21.1 & 10.7 & 15.4\end{array}$ $29.411 .5 \quad 20.3$ 31.111 .920 .0 $28.3 \quad 10.9 \quad 19.7$ $\begin{array}{llll}28.3 & 10.3 & 18.4\end{array}$ $\begin{array}{lll}30.6 & 10.8 & 20.5\end{array}$ $23.9 \quad 10.9 \quad 17.3$ $28.3 \quad 11.1 \quad 24.5$ $\begin{array}{lll}31.1 & 10.3 \quad 20.6\end{array}$ $\begin{array}{lll}35.6 & 11.5 & 24.7\end{array}$

$\begin{array}{ll}4.8 & 22.6 \\ 6.4 & 27.6 \\ 7.0 & 27.3 \\ 6.4 & 26.9 \\ 7.1 & 25.7 \\ 7.1 & 27.7 \\ 5.4 & 24.5 \\ 5.1 & 31.7 \\ 7.1 & 32.0 \\ 7.4 & 27.9\end{array}$

\section{Wirt County}

$\begin{array}{lllll}404 & 7 & 20 & 59 & 39.0492\end{array}$

$404 \quad 120 \quad 60 \quad 39.0492$

$\begin{array}{lllll}628 & 5 & 31 & 73 & 38.9962\end{array}$

$\begin{array}{llll}629 & 6 & 10 & 73\end{array}$

38.9691

81.2765
81.2765
81.2779

272.

$273.4 \quad 588.6$

$\begin{array}{rrr}272.8 & 273.4 & 1068.0 \\ 307.8 & 310.3 & 670.9\end{array}$

$\begin{array}{rrr}16.1 & 12.1 & 6.7\end{array}$

$28.911 .8 \quad 25.4$

$\begin{array}{llllll}198.2 & 201.2 & 564.5 & 25.6 & 12.9 & 22.3\end{array}$
81.2786
Wood County

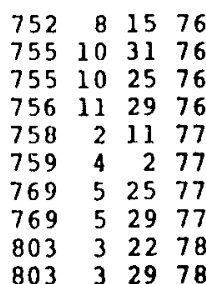

39.2602 39.2485 39.2485 39.0806 39.2628 39.2392

39.2519

39.2519

39.1157

39.1157
81.6780 81.6720 81.6720 81.5085 81.6628 81.6695 81.6901 81.6901 81.4947 81.4947 $\begin{array}{lll}193.5 & 197.8 & 1260.7\end{array}$ $217.3 \quad 220.4 \quad 1285.0$ $211.2 \quad 221.3 \quad 4045.3$ $235.6 \quad 239.0 \quad 1309.1$ $201.2 \quad 204.8 \quad 1285.0$

$\begin{array}{llll}188.4 & 190.2 \quad 577.0\end{array}$ $188.4 \quad 190.2 \quad 1309.1$ $\begin{array}{llll}219.2 & 222.5 & 677.3\end{array}$ $\begin{array}{llll}219.2 & 222.5 & 1935.8\end{array}$ $\begin{array}{llll}43.3 & 12.6 \quad 24.3\end{array}$ $49.412 .4 \quad 20.6$ $\begin{array}{lll}46.7 & 12.4 & 26.6\end{array}$ $85.012 .6 \quad 17.9$ $38.3 \quad 12.2 \quad 19.9$ $40.012 .6 \quad 21.3$ $\begin{array}{lll}25.6 & 12.7 & 22.2\end{array}$ $\begin{array}{lll}35.6 & 12.7 & 17.4\end{array}$ $28.912 .6 \quad 24.1$ 60.012 .624 .5

$\begin{array}{rr}9.2 & 31.6 \\ 12.7 & 27.7 \\ 9.3 & 33.9 \\ 18.6 & 22.5 \\ 9.5 & 27.2 \\ 9.3 & 28.6 \\ 4.1 & 29.3 \\ 9.5 & 24.7 \\ 4.8 & 31.3 \\ 13.5 & 31.5\end{array}$


Wyoming County

\begin{tabular}{|c|c|c|c|c|c|c|c|c|c|c|c|c|c|}
\hline $\begin{array}{c}\text { WELI, } \\
\text { NO }\end{array}$ & & & 12 & $\begin{array}{l}\text { LATITUDE } \\
\text { DEGREES }\end{array}$ & $\begin{array}{l}\text { LONG ITUDE } \\
\text { DEGREES }\end{array}$ & $\begin{array}{c}\text { ELEV } \\
M\end{array}$ & $\underset{M}{\text { RBELV }}$ & $\underset{M}{\text { DEPTH }}$ & $\begin{array}{c}\mathrm{BHT} \\
\mathrm{C}\end{array}$ & $\begin{array}{l}\text { STEMP } \\
\text { C }\end{array}$ & $\begin{array}{c}\text { GRADI ENT } \\
C / R M\end{array}$ & $\begin{array}{c}\text { BHTCOR } \\
\text { C }\end{array}$ & $\begin{array}{c}\text { AAPGRAD } \\
\text { C/RM }\end{array}$ \\
\hline $\begin{array}{l}105 \\
106 \\
175 \\
222 \\
255 \\
277 \\
314 \\
379 \\
436 \\
783\end{array}$ & $\begin{array}{r}8 \\
5 \\
7 \\
7 \\
6 \\
8 \\
4 \\
3 \\
1 \\
10\end{array}$ & $\begin{array}{r}13 \\
28 \\
29 \\
8 \\
13 \\
18 \\
3 \\
18 \\
5 \\
14\end{array}$ & $\begin{array}{l}71 \\
70 \\
78 \\
65 \\
66 \\
67 \\
73 \\
64 \\
66 \\
77\end{array}$ & $\begin{array}{l}37.5442 \\
37.5345 \\
37.6965 \\
37.7629 \\
37.7705 \\
37.7696 \\
37.7650 \\
37.7051 \\
37.6659 \\
37.5282\end{array}$ & $\begin{array}{l}81.6432 \\
81.6273 \\
81.6031 \\
81.4464 \\
81.4632 \\
81.4733 \\
81.4974 \\
81.6673 \\
81.7346 \\
81.2911\end{array}$ & $\begin{array}{l}475.8 \\
570.9 \\
455.7 \\
805.3 \\
798.9 \\
851.0 \\
553.5 \\
580.6 \\
393.8 \\
738.8\end{array}$ & $\begin{array}{l}75.8 \\
71.2 \\
58.7 \\
05.9 \\
99.8 \\
51.3 \\
53.8 \\
81.6 \\
94.1 \\
41.9\end{array}$ & $\begin{array}{r}896.1 \\
1066.8 \\
877.5 \\
1165.3 \\
1125.0 \\
1178.4 \\
1028.7 \\
1221.0 \\
1112.5 \\
1207.3\end{array}$ & $\begin{array}{l}30.0 \\
30.6 \\
26.7 \\
43.3 \\
35.6 \\
36.1 \\
35.0 \\
36.1 \\
33.9 \\
33.9\end{array}$ & $\begin{array}{r}12.0 \\
11.1 \\
12.0 \\
8.5 \\
8.5 \\
8.0 \\
11.0 \\
10.8 \\
12.6 \\
9.4\end{array}$ & $\begin{array}{l}20.1 \\
18.2 \\
16.7 \\
29.9 \\
24.0 \\
23.8 \\
23.4 \\
20.8 \\
19.1 \\
20.3\end{array}$ & $\begin{array}{l}6.5 \\
7.8 \\
6.4 \\
8.5 \\
8.2 \\
8.6 \\
7.5 \\
8.9 \\
8.1 \\
8.8\end{array}$ & $\begin{array}{l}27.3 \\
25.5 \\
24.0 \\
37.2 \\
31.3 \\
31.1 \\
30.7 \\
28.0 \\
26.4 \\
27.5\end{array}$ \\
\hline
\end{tabular}


WEST VIRGINIA GRAVITY DATA

PRINCI PAL FACTSATAGAVITY STATION

STA NAME AND NUM

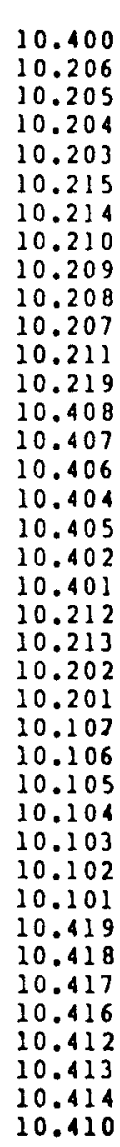

\section{LATITUDE
NORTH +}

3848.6

$38 \quad 54.53$

3855.31

$38 \quad 56.00$

$38 \quad 57.69$

$\begin{array}{ll}38 & 57.13 \\ 38 & 57.15\end{array}$

3857.15

3858.28

$38 \quad 58.86$

3858.86

3848.81

$38 \quad 48.50$

$38 \quad 47.72$

$\begin{array}{lll}38 & 46.10\end{array}$

$\begin{array}{lll}38 & 46.40\end{array}$

$38 \quad 45.68$

$\begin{array}{ll}38 & 47.39 \\ 38 & 48.17\end{array}$

$\begin{array}{ll}38 & 48.17 \\ 38 & 59.55\end{array}$

3859.83

$38 \quad 57.47$

$\begin{array}{ll}38 & 56.15 \\ 38 & 56.36\end{array}$

$38 \quad 56.71$

$\begin{array}{ll}38 & 56.71 \\ 38 & 57.15\end{array}$

$38 \quad 56.02$

$\begin{array}{ll}38 & 55.50 \\ 38 & 55.02\end{array}$

3855.02

$38 \quad 52.31$

3851.70

3850.98

3850.58

$38 \quad 50.28$

3850.17

$38 \quad 49.88$

$\begin{array}{ll}38 & 49.43 \\ 38 & 40.50\end{array}$
LONG ITUDE
EAST +

$\begin{array}{rr}-80 & 52.48 \\ -80 & 45.07\end{array}$

$\begin{array}{ll}-80 & 45.07 \\ -80 & 47.00\end{array}$

$-8048.18$

$\begin{array}{lll}-80 & 49.23\end{array}$

$\begin{array}{lll}-80 & 49.62\end{array}$

$\begin{array}{ll}-80 & 48.30 \\ -80 & 47.55\end{array}$

$\begin{array}{ll}-80 & 47.55 \\ -80 & 46.39\end{array}$

$\begin{array}{rr}-80 & 45.68\end{array}$

$\begin{array}{ll}-80 & 45.29 \\ -80 & 49.50\end{array}$

$\begin{array}{lll}-80 & 47.15\end{array}$

$\begin{array}{lll}-80 & 47.05\end{array}$

$\begin{array}{lll}-80 & 47.82\end{array}$

$\begin{array}{rr}-80 & 49.53 \\ -80 & 49.25\end{array}$

$\begin{array}{ll}-80 & 49.25 \\ -80 & 48.38\end{array}$

$-8050.83$

$\begin{array}{ll}-80 & 52.47\end{array}$

$-8048.30$

$\begin{array}{rr}-80 & 48.98\end{array}$

$-8051.00$

$\begin{array}{rl}-90 & 45.07\end{array}$

$\begin{array}{rr}-80 & 47.05 \\ -80 & 48.07\end{array}$

$\begin{array}{ll}-80 & 48.87\end{array}$

$\begin{array}{lll}-80 & 50.12\end{array}$

$\begin{array}{ll}-80 & 51.45 \\ -80 & 51.83\end{array}$

-80
-80
-80.08

$\begin{array}{rl}-80 & 50.52\end{array}$

$-80 \quad 50.59$

$\begin{array}{rl}-80 & 49.19\end{array}$

$\begin{array}{rr}-80 & 47.77\end{array}$

$\begin{array}{ll}-80 & 47.20 \\ -80 & 46.42\end{array}$

$\begin{array}{rr}-80 & 46.42 \\ -80 & 47.49\end{array}$

$\begin{array}{rr}-80 & 47.49 \\ -80 & 47.78\end{array}$
ELEVATION

FEET

739.000

733.000
745.000

745.000

740.000

747.000

759.000

759.000

810.000

840.000

882.000

820.000

826.000

868.000

86.000

900.000

847.000

824.000

736.000

736.000

772.000

772.000

731.000

727.000

723.000

719.000

719.000

720.000

770.000

780.000

790.000

800.000

825.000

848.000

824.000
996.000
TERR

OBSERVE

$0.000 \quad 979967.140$

.620979983 .223

$.670 \quad 979903.190$

$670 \quad 979982.825$

$.380 \quad 979982.687$

$.110 \quad 979983.334$

$.410 \quad 979985.034$

$1.440 \quad 979983.334$

$1.200 \quad 979982.620$

$1.100 \quad 979981.770$

$100 \quad 979976.468$

979968.204

$20 \quad 979968.696$

$170 \quad 979961.578$
.500

$.500 \quad 979962.65$

$1.220 \quad 979957.768$

$2.170 \quad 979963.471$

$.500 \quad 979965.808$

$.990 \quad 979985.004$

$.530 \quad 979987.849$

$.620 \quad 979982.493$

$2.200 \quad 979980.782$

.900

979981.157

1.000

.690
.480

979982.464

979981.015

979981.015

.680

.370

.380

.850

(3)

(1)

97975.227

979972.996

.270

.990

(1)

979972.385

$1.360 \quad 979970.91$

$1.350 \quad 979968.997$

$0.000 \quad 979940.700$
REE AIR

BOUGUER

THEOR G

MGAL

C BOUGUER

MGAL

$-21.484$

$-48.599 \quad 980063.403$

$-44.606 \quad 980072.135$

$-46.148 \quad 980073.285$

$-47.248 \quad 980074.301$

$-48.971980076 .792$

$-46.147980075 .966$

$-46.745 \quad 980075.996$

$-46.062980075 .981$

$-46.331980077 .514$

$-49.168090078 .516$

$-46.346 \quad 980063.712$

$-45.038000063 .256$

$-45.671980062 .10$

$-45.027 \quad 980059.723$
-46.556

$-46.516 \quad 980060.165$

$-47.378 \quad 980059.105$

$-47.369 \quad 980061.62$

$\begin{array}{lll}-47.559 & 980062.770\end{array}$

$-48.065980079 .533$

$-47.971980079 .946$

$-49.908 \quad 980076.467$

$-47.455 \quad 980074.52$

$-49.848 \quad 980074.83$

-49.297980075.347

$-51.633 \quad 980075.996$

-49.968 980074.331

$-50.63390073 .564$

$-50.79498072 .057$

$-50.58298072 .062$

-47.473 98068.865

$-48.74690067 .967$

$-46.569000060318$

$-46.11900005 .316$

$-45.33800005 .714$

$-45.449980065 .287$

45.465980064 .625

$-17.101$

$-51.071$

980051.486

$-48.599$

$-43.986$

$-45.478$

$-45.330$

$-46.868$

-47.861
-44.857

$-46.335$

$-44.622$

$-45.131$

$-45.284$

$-45.446$

$-43.818$

$-44.501$

$-45.526$

$-46.158$

$-45.199$

$-47.059$

$-47.075$

$-47.441$

$-46.255$

$-48.948$

$-50.943$

$-49.488$

$-49.953$

$-50.424$

$-46.623$

$-48.456$

$-46.299$

$-45.129$

$-43.978$

$-44.099$

$-44.635$

$-51.071$ 
PRINCIPAL FACTSATARAVITY STATION

STA NAME AND NUM

9.105
9.102
9.1101
9.100
9.104
9.1105
9.107
9.108
9.109
9.110
9.111
9.112
9.113
9.114
8.211
8.212
8.213
8.214
8.215
9.216
8.217
10.400
10.306
10.307
10.308
10.305
10.304
10.304
10.302
10.301
10.319
10.318
10.317
10.316
10.315
10.314
10.313
10.312
10.311
10.310
LATITUDE
NORTI +

$38 \quad 39.73$

3839.50

$38 \quad 37.61$

3839.99

3840.40

38 3 40.53

3841.05

38411.05

$\begin{array}{ll}38 & 42.31 \\ 38 & 43.38\end{array}$

$38 \quad 44.36$

3844.78

$38 \quad 42.42$

$\begin{array}{lll}38 & 43.16\end{array}$

$\begin{array}{lll}38 & 13.89\end{array}$

$\begin{array}{lll}38 & 44.35\end{array}$

3844.85

3844.04

3843.67

3849.00

3848.28

$\begin{array}{ll}38 & 47.90 \\ 38 & 49.38\end{array}$

3850.08

3851.07

3851.03

3850.65

$38 \quad 49.68$

$38 \quad 48.85$

3848.13

$\begin{array}{ll}38 & 47.05 \\ 38 & 46.03\end{array}$

38046.8

$\begin{array}{lll}38 & 45.22\end{array}$

$\begin{array}{ll}38 & 45.97\end{array}$
EAST +

$-80 \quad 43.05$

$\begin{array}{ll}-80 & 42.19\end{array}$

$-8042.39$

0042.04

$-8043.05$

$80 \quad 42.84$

$80 \quad 40.05$

$-8038.98$

$-8039.56$

39.00

$-8039.52$

8045.86

$-8046.16$

$\begin{array}{lll}-80 & 46.22\end{array}$

$-80 \quad 53.55$

.8053 .48

$-8054.10$

$-8055.75$

$-8056.23$

$\begin{array}{ll}-80 & 57.84 \\ -80 & 59.22\end{array}$

-80 58.77

$-8058.56$

$-8057.37$

$\begin{array}{ll}-80 & 55.83 \\ -80 & 55.93\end{array}$

\section{EET}

850.000

1055.000

1367.000

850.000

850.000

(350.000

0.000

1238.000

1260.000

265.000
1110.000

1071.000

940.000

907.000

965.000

1140.000

1009.000

949.000

916.000

1212.000

1002.000
795.000

825.000

869.000

775.000

759.000

761.000

761.000

(7).000

730.000

720.000

723.000

736.000

736.000

720.000

764.000

774.000

883.000

777.000

\section{MGAL}

MGAL

PRE

.550
.490

979951.795

.560979952 .070

$.360 \quad 979952.732$

$.520 \quad 979952.726$

.180 979952.729

$.120 \quad 979929.836$

$.100 \quad 979929.376$

$1.000 \quad 979929.378$

$1.050 \quad 979944.665$

$.090 \quad 979953.995$

$.950 \quad 979956.742$

$1.360 \quad 979949.311$

$.500 \quad 979942.636$

$1.340 \quad 979949.089$

$1.280 \quad 979954.176$

$1.450 \quad 979956.262$

.840979938 .796

$.930 \quad 979952.165$

$1.230 \quad 979967.969$

$1.410 \quad 979962.897$

$1.050 \quad 979962.88$

$1.200 \quad 979971.332$

1.480979970 .194

$1.470 \quad 979972.298$

$1.810 \quad 979972.250$

$1.800 \quad 979973.197$

$1.810 \quad 979972.426$

$1.790 \quad 979971.925$

$1.750 \quad 979970.317$

1.260979968 .012

$1.220 \quad 979967.204$

$1.700 \quad 979966.325$

$1.850 \quad 979963.484$

$.830 \quad 979958.090$
$1.350 \quad 979965.099$

$1.750 \quad 979965.099$
$-18.606$ $-2.067$ $-14.567$

.

$-4.401$

$-3.928$

$-9.211$

$-14.751$

$-15.725$

$-14.229$

$-12.474$

$-13.709$

$-15.462$

$-9.894$

$-21.48$

$-18.422$

$-19.532$

$-19.532$

$-23.996$

$-23.161$

$-26.139$

$-25.330$

$-25.344$

$-25.448$

-25.470
-24.688

$-26.748$

$-23.817$

$-22.392$

-18.386
-22.789
BOUGUER THEOR G T C BOUGUER

$-17.597980050 .354$

$\begin{array}{lll}-53.916 & 900050.015 & -47.047\end{array}$

$-48.692 \quad 980050.015-53.426$

-43.558 $900047.337=47.192$

$-47.702900050 .736 \quad-47.342$

-4.00051.339 -47.331

$-47.65190051 .3390-47.131$

$-47.79500051 .486-47.615$

-47.470 $900051.530-47.350$

-47.376 980052.295 $=47.276$

$-47.07490052 .295-46.074$

$-47.070 \quad 980054.148 \quad-46.890$

$-46.846 \quad 980055.722-45.796$

$-46.812980057 .163 \quad-46.722$

$-46.661 \quad 980057.781 \quad-45.711$

-47.143 980054.309 -45.783

$-44.414 \quad 980055.398-43.914$

$-46.809 \quad 980056.472-45.549$

$-46.076 \quad 980057.148 \quad-44.796$

$-46.704 \quad 980057.884 \quad-45.254$

$\begin{array}{lll}-45.232 & 980056.692 & -44.392\end{array}$

$\begin{array}{lll}-43.909 & 980056.248 & -42.979\end{array}$

$\begin{array}{lll}-48.599 & 980063.403 & -46.279\end{array}$

$-46.560 \quad 980063.992 \quad-45.330$

$-47.994 \quad 980062.932 \quad-46.584$

$-48.524 \quad 980062.373 \quad-47.474$

$-46.754 \quad 980064.551 \quad-45.554$

$-49.883 \quad 980065.582 \quad-48.403$

$-49.116 \quad 980067.039 \quad-47.646$

$-50.144 \quad 980066.981 \quad-49.334$

$-50.662 \quad 980066.966 \quad-48.062$

$\begin{array}{lll}-49.229 & 980066.421 & -48.419\end{array}$

$-49.901 \quad 980064.993 \quad-48.111$

$-50.107 \quad 980063.771-48.357$

$-50.573 \quad 980062.711 \quad-49.313$

$-49.791 \quad 980061.121 \quad-48.571$

$-51.305 \quad 980060.798-49.605$

$-49.875 \quad 980059.164-48.025$

$-48.791 \quad 980058.429-46.991$

$\begin{array}{lll}-48.502 & 980059.532 & -47.152 \\ -49.291 & 980060.974 & -47.541\end{array}$ 
PRINCIPAL FACTSAT GRAVITY STATIONG

STA NAME AND NUM

$\begin{array}{lr}10.309 & 79 \\ 9.104 & 80 \\ 9.201 & 81 \\ 9.202 & 82 \\ 9.203 & 83 \\ 9.204 & 84 \\ 9.295 & 85 \\ 9.211 & 86 \\ 9.212 & 87 \\ 9.213 & 88 \\ 9.214 & 89 \\ 9.215 & 90 \\ 9.216 & 91 \\ 9.207 & 93 \\ 9.208 & 94 \\ 9.210 & 95 \\ 9.209 & 96 \\ 9.316 & 97 \\ 9.315 & 98 \\ 9.314 & 99 \\ 9.313 & 100 \\ 9.317 & 101 \\ 9.318 & 102 \\ 9.319 & 103 \\ 9.306 & 104 \\ 9.307 & 105 \\ 9.308 & 106 \\ 9.309 & 107 \\ 9.319 & 108 \\ 9.311 & 109 \\ 9.312 & 110 \\ 9.305 & 111 \\ 9.304 & 112 \\ 9.303 & 113 \\ 9.302 & 114 \\ 9.301 & 115 \\ 6.101 & 116 \\ 6.102 & 117 \\ 6.103 & 118 \\ 6.105 & 119 \\ 9 & \end{array}$

LATITUD

NORTH +

\section{$38 \quad 46.98$}

$38 \quad 39.99$

38 4 31.93

$38 \quad 41.65$

3841.94

$38 \quad 41.13$

$38 \quad 42.98$

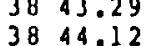

3844.81

$38 \quad 44.66$

$\begin{array}{lll}38 & 44.73\end{array}$

$\begin{array}{lll}38 & 41.07\end{array}$

$38 \cdot 40.33$
38
38

$38 \quad 39.18$

$\begin{array}{ll}38 & 39.81 \\ 38 & 37.03\end{array}$

(3) 36.15

$38 \quad 35.2$

$38 \quad 34.87$

$38 \quad 34.31$

3834.7

3834.36

3834.31

3834.6

3834.59

3833.93

$\begin{array}{ll}38 & 33.97 \\ 38 & 33.65\end{array}$

3832.8

$\begin{array}{lll}38 & 32.2\end{array}$

$\begin{array}{ll}38 & 31.35 \\ 38 & 30.77\end{array}$

$38 \quad 30.77$

3829.2

$\begin{array}{lll}38 & 28.39\end{array}$

3827.55

\section{LONG ITUDE}

FEET.

$\begin{array}{rr}-60 & 55.08\end{array}$ .05 00030.02 0035.62 $-8035.19$ $-8034.65$ 8034.46 $-8034.81$ $\begin{array}{ll}-80 & 35.51\end{array}$ $\begin{array}{ll}-80 & 35.90\end{array}$ $-80.34 .03$ $-80 \quad 33.58$
-80 .80 30.07 80.50 $\begin{array}{lll}80 & 42.27\end{array}$ 42.27 $-8042.30$ 42.62 $\begin{array}{lll}-80 & 41.46\end{array}$

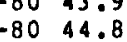
$\begin{array}{rr}-80 & 41.95\end{array}$ $\begin{array}{lll}-80 & 41.46\end{array}$ $\begin{array}{lll}-80 & 40.43\end{array}$ $\begin{array}{ll}-80 & 39.69\end{array}$ $\begin{array}{ll}-80 & 39.24\end{array}$ $-8038.97$ $-8038.00$ $\begin{array}{rl}-80 & 41.96\end{array}$ $\begin{array}{lll}-80 & 42.35\end{array}$ $\begin{array}{rr}-80 & 42.83\end{array}$ $\begin{array}{ll}-80 & 43.67\end{array}$ $\begin{array}{lll}-80 & 44.17\end{array}$ $-80 \times 4.10$ $\begin{array}{ll}-80 & 43.90\end{array}$ $\begin{array}{ll}-80 & 43.51 \\ -80 & 43.12\end{array}$

800.000
839.000
1320.000
1320.000
1315.000
1440.000
1640.000
1200.000
920.000
850.000
870.000
1051.000
1123.000
1044.000
1006.000
1054.000
1020.000
1494.000
1180.000
1140.000
1118.000
1108.000
1076.000
1071.000
1150.000
1165.000
1170.000
1220.000
1246.000
1325.000
1340.000
1239.000
1640.000
1824.000
1527.000
1330.000
1133.000
1164.000
1242.000
1331.000

TERR CORR MGNi MCAL MGNL BOUGUER THEOR G MGAL.

T C ROUGUER MGAL

$1.680 \quad 979971.095$ $1.130 \quad 979952.391$ $.460 \quad 979922.719$ $1.130 \quad 97922.711$ 1.120979925 .768 $1.950 \quad 979905.529$ 1.950 979905.529 $.380 \quad 97932.965$ $.290 \quad 979952.145$ $.270 \quad 979958.005$

$.280 \quad 979960.615$

$.530 \quad 979946.946$

.310979943 .251

.330979938 .192

$.320 \quad 979939.028$

$.340 \quad 979939.042$

.320979939 .056

$.920 \quad 979908.12$

$.740 \quad 979924.410$

$.720 \quad 979925.122$

$.700 \quad 979925.558$

$.700 \quad 979925.293$

$.690 \quad 979929.070$

$.670 \quad 979929.31$

$.670 \quad 979922.525$

$.730 \quad 979920.970$

$.860 \quad 979922.526$

$.770 \quad 979918.545$

$.780 \quad 979916.680$

$.830 \quad 979913.337$

$.840 \quad 979909.905$

$.780 \quad 979915.170$

$1.030 \quad 979891.355$

$1.140 \quad 979879.827$

$.960 \quad 979897.893$

$.840 \quad 979906.966$

$2.540 \quad 979915.93$ $2.600 \quad 979912.709$

$2.750 \quad 979905.897$

$-14.675$ $-19.428$ . 7.718 1.718 7.378 $-9.294$ $-16.908$ $-18.853$ $-15.376$

$-11.800$

$-8.826$

$-15.932$

$-17.582$

$-11.362$

2.272
-9.688

$-9.688$

$-11.402$

-12.491
-12.874

$-12.754$

$-12.410$

$-11.383$

$-11.836$

$-9.616$

$-8.91$

$-3.861$

$-5.940$

$-9.705$

12.078

3.485

$-5.120$

$-12.963$

$-12.700$

$-10.971$

$-8.092$

$-40.280$

$\begin{array}{rrr}-41.960 & 980061.018 & -40.280 \\ -48.044 & 980050.736 & -46.914\end{array}$ $\begin{array}{lll}-51.731 & 980053.589 & -51.271\end{array}$ $\begin{array}{lll}-52.091 & 980053.942 & -50.961\end{array}$ $-48.569 \quad 980053.177 \quad-47.449$
-50.596 $-40.558000052 .412-46.608$ $-40.558090052 .4120-46.608$ $-40.222080055 .133-49.842$ $-48.286 \quad 980055.589-47.996$ $-45.044 \quad 980056.810-47.574$

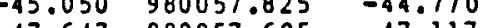
$-47.647 \quad 980057.605 \quad-47.117$ $-47.6428980057 .708-46.818$ -47.5280
$-51.540052 .324-51.210$

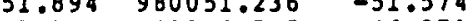
$-47.311 \quad 980049.545-46.971$ $-50.262 \quad 980050.471 \quad-49.942$ $\begin{array}{lll}-40.664 & 980046.384 & -47.764\end{array}$ $\begin{array}{lll}-49.935 & 980045.091 & -49.195\end{array}$ $\begin{array}{lll}-49.935 & 980045.091 & -49.195\end{array}$ $\begin{array}{lll}-50.623 & 980043.210 & -49.923\end{array}$ $\begin{array}{lll}-50.623 & 980043.210 & -49.923\end{array}$ $\begin{array}{lll}-49.453 & 980043.034 & -48.763\end{array}$ $\begin{array}{lll}-48.939 & 980042.461 & -48.7639\end{array}$ $\begin{array}{lll}-50.606 & 980042.079 & -49.936\end{array}$ $-51.571 \quad 980042.387 \quad-50.841$ $-49.730 \quad 980042.402$
-51.227 $\begin{array}{lll}-51.227 & 980042.916 & -50.457\end{array}$

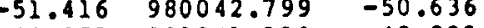
$\begin{array}{lll}-51.0416 & 980042.799 & -50.636\end{array}$ $-51.644 \quad 980041.888 \quad-50.804$ $-51.964 \quad 980041.418 \quad-51.284$ $\begin{array}{lll}-50.489 & 980040.169 & -49.459\end{array}$ $\begin{array}{lll}-50.133 & 980039.317 & -48.993\end{array}$ $\begin{array}{lll}-40.597 & 980038.039 & -47.637\end{array}$ $\begin{array}{lll}-50.482 & 980037.187 & -49.642\end{array}$ $\begin{array}{lll}-51.607 & 980035.469 & -49.067\end{array}$ $\begin{array}{lll}-52.401 & 980034.897-49.801\end{array}$ $\begin{array}{lll}-53.332 & 980033.693 & -50.582 \\ -53.489 & 980032.460 & -50.539\end{array}$ 
PRINCIPAL PACISTATGRAVITY STATIONS

GTA NAME AND NUM

$\begin{array}{ll}6.104 & 120 \\ 6.106 & 121 \\ 9.402 & 122 \\ 9.403 & 123 \\ 9.404 & 124 \\ 8.210 & 125 \\ 8.209 & 126 \\ 8.208 & 127 \\ 8.207 & 128 \\ 8.206 & 129 \\ 8.210 & 130 \\ 8.219 & 131 \\ 8.218 & 132 \\ 8.205 & 133 \\ 8.204 & 134 \\ 8.203 & 135 \\ 8.202 & 136 \\ 8.201 & 137 \\ 8.313 & 138 \\ 8.312 & 139 \\ 8.311 & 140 \\ 8.310 & 141 \\ 8.309 & 142 \\ 8.308 & 143 \\ 8.307 & 144 \\ 8.306 & 145 \\ 8.305 & 146 \\ 8.304 & 147 \\ 8.303 & 148 \\ 8.302 & 149 \\ 8.301 & 150 \\ 8.325 & 151 \\ 8.326 & 152 \\ 8.327 & 153 \\ 8.328 & 154 \\ 8.212 & 155 \\ 8.213 & 156 \\ 8.101 & 157 \\ 8.102 & 158 \\ 8.103 & 159\end{array}$

LATITUDE LONGITUDE
EAST +

3827.97

$\begin{array}{lll}38 & 27.25\end{array}$

30 .

38 36.88

$\begin{array}{ll}38 & 40.99\end{array}$

$38 \quad 39.93$

38 30.10

3840.45

3841.61

38 . 42.81

$38 \quad 39.88$

3839.25

$38 \quad 39.31$

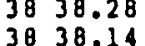

3838.14

3836.45

38 35.81

3835.12

3833.97

$38 \quad 34.58$

$38 \quad 33.13$

$\begin{array}{lll}38 & 34.98 \\ 38 & 34.12\end{array}$

$\begin{array}{lll}38 & 34.12 \\ 38 & 33.97\end{array}$

3 B 33,46

$38 \quad 32.75$

$\begin{array}{lll}38 & 32.99\end{array}$

3835.52

$38 \quad 35.85$

$\begin{array}{lll}38 & 36.63\end{array}$

$\begin{array}{lll}38 & 37.23\end{array}$

3838.91

3839.57

$38 \quad 40.40$

$\begin{array}{ll}38 & 41.11 \\ 38 & 41.04\end{array}$

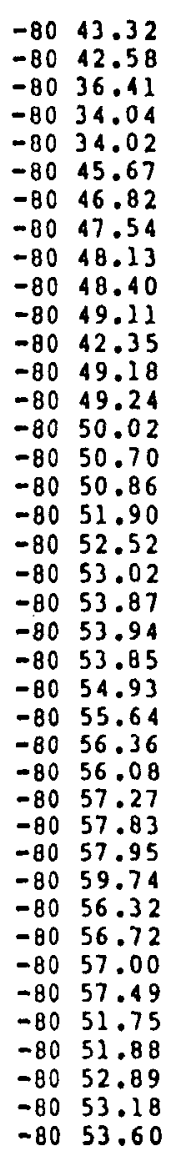

\section{EEET} MGAL

OASERVED
MGAL

PREE A
MGN

BOUGUER
MGAL

THEOR
MGN

1295.000
1401.000

1691.000

1065.000

1010.000
821.000

821.000
834.000

820.000

840.000

897.000

1060.000

2431.000

1430.000

830.000

830.000

840.000

845.000

821.000

810.000

852.000

807.000

810.000

807.000

807.000

794.000

780.000

792.000

792.000

785.000

805.000

772.000

790.000

840.000

885.000

870.000

840.000

910.000

988.000

930.000
911.000
$2.870 \quad 979$

$.330 \quad 979894.925$

.

080 979

.480097930 .599

40097930.141

$390 \quad 979952.736$

$440 \quad 979954.098$

$.250 \quad 979953.136$

$.290 \quad 979950.679$

.200

$590 \quad 979922.004$

$.170 \quad 979952.848$

$.120 \quad 979953.011$

.340979953 .769

$.140 \quad 979951.886$

.260979952 .338

$.100 \quad 979950.090$

$.110 \quad 979946.925$

$.900 \quad 979948.681$

$.160 \quad 979947.756$

$.180 \quad 979946.757$

$.120 \quad 979948.003$

$.200 \quad 979947.539$

$.190 \quad 979948.690$

$.210 \quad 979947.853$

$.220 \quad 979947.889$

$.210 \quad 979946.494$

.250979945 .354

$.240 \quad 979947.222$

$.290 \quad 979949.440$

$.310 \quad 979949.567$

$.100 \quad 979946.584$

$.130 \quad 979946.945$

$.150 \quad 979952.584$

$.170 \quad 979949.361$

.330979945 .748

.350
$.270 \quad 979949.960$
$670 \quad 979922.208$
$-9.341$

-6.100
6.712

$-16.139$

$-18.021$

$-19.994$

$-19.669$

$-17.765$

$-16.360$

2.780

1.629

$-19.655$

$-16.956$

$-16.854$

$-18.454$

$-19.252$

$-17.526$

$-18.989$

$-19.029$

$-19.223$

$-18.874$

-18.430
-21.314

$-21.314$

$-19.502$

$-20.806$

$-19.022$

$-20.610$

$-16.071$

$-15.968$

$-17.553$

$-15.161$

$-12.658$

$-15.714$

\section{$-53.510$}

980033.076

(1)

. 2.46390046 .914

200050

47.99690052 .206

$-47.637980050 .898$

$-46.415980049 .912$

$-46.954980051 .412$

46.954

4.028

980054.030

980054.883

980050.574

$-46.875980049 .648$

$-45.606980049 .736$

$-45.674980048 .222$

$-46.456980048 .016$

-46.879 980045.532

$-46.585980044 .591$

$-46.514980043 .577$

$-46.656980042 .975$

$-46.748 \quad 980041.888$

$-46.398 \quad 980042.784$

$-45.511980040 .654$

$-47.91798004 .372$

$-46.771980042 .108$

$-46.515980041 .888$

$-47.581980041 .139$

$-46.478980040 .096$

$-46.941980040 .448$

$-47.361980044 .165$

$-46.153 \quad 980045.797$

$-46.153908049$

$-46.198980050 .118$

$-46.198900051 .33$

$-46.666980052 .383$

980052.280
T C BOUGUER

HCNL

$-50.640$

$-50.554$

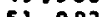

(1)

$-47.576$

-520
-4.5207

$-46.165$

$-46.165$

$-15.007$

$-45.358$

$-47.794$

$-46.755$

$-45.266$

$-46.196$

$-46.779$

$-46.475$

$-45.614$

$-46.496$

$-46.278$

$-45.311$

$-47.727$

$-46.295$

$-47.371$

$-46.701$

$-47.071$

$-46.053$

$-47.443$

$-46.053$

$-46.028$

$-46.026$

$-46.316$

$-46.515$ 
PRINCIPAL PACTSATERAVITY STATIONG

STA NAME AND NUM

LATITUDE

NORTH +

$38 \quad 40.11$

38 (1). 41.29

30 . 42.98

3843.82

$\begin{array}{ll}38 & 44.29 \\ 38 & 44.89\end{array}$

3840.80

$\begin{array}{lll}38 & 39.97\end{array}$

3839.22

3838.79

$\begin{array}{lll}38 & 38.81 \\ 38 & 37.81\end{array}$

$\begin{array}{lll}38 & 37.46\end{array}$

3836.93

$38 \quad 36.50$

35.65

3835.67

3835.03

3834.50

30.81

3831.43

8 30.60

3831.02

3830.62

3830.00

3831.56

$38 \quad 30.97$

उ8 29.84

$\begin{array}{ll}38 & 29.29 \\ 38 & 29.00\end{array}$

$\begin{array}{ll}38 & 28.70\end{array}$

$\begin{array}{lll}38 & 28.39\end{array}$

3827.98

$\begin{array}{ll}38 & 27.29 \\ 38 & 26.78\end{array}$
LONG ITUD
EAST +

$\begin{array}{ll}-80 & 54.26\end{array}$

$\begin{array}{ll}-80 & 55.13 \\ -80 & 55.17\end{array}$

$-80 \quad 55.49$

$\begin{array}{ll}-80 & 55.90 \\ -80 & 56.54\end{array}$

$\begin{array}{lll}-80 & 56.54\end{array}$

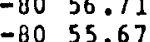

$-80 \quad 55.63$

$-80 \quad 54.92$

$-80 \quad 54.49$

$\begin{array}{rr}-80 & 53.54\end{array}$

-30
-82.62

$-8050.69$

$-80 \quad 49.68$
-80

$\begin{array}{lll}-80 & 48.94\end{array}$

$\begin{array}{lll}-80 & 49.18\end{array}$

40.64

-80
-88.49

$-8048.10$

$-8048.35$

$-80 \quad 48.38$

-80
-80 4.78

$\begin{array}{lll}-80 & 45.91\end{array}$

$\begin{array}{ll}-80 & 45.28 \\ -80 & 57.35\end{array}$

$\begin{array}{ll}-80 & 57.35 \\ -80 & 58.48\end{array}$

$-80 \quad 59.28$

$\begin{array}{lll}-80 & 45.38\end{array}$

$-8045.50$

$\begin{array}{rr}-80 & 45.89\end{array}$

$\begin{array}{lll}-80 & 46.45\end{array}$

$\begin{array}{lll}-80 & 46.55\end{array}$

$\begin{array}{ll}-80 & 46.60 \\ -80 & 46.97\end{array}$
ELEVATION

FEET

875.000

835.000

820.000

796.000

800.000

790.000

790.000
930.000

930.000

950.000

915.000

908.000

1232.000

1232.000

840.000

818.000

940.000

940.000

127.000

1236.000

1195.000

1195.000

1000.000

1000.000

1530.000

1530.000

1490.000

140.000

1123.000

1111.000

1320.000

1120.000

1152.000

1168.000

1217.000

1280.000

1380.000

1872.000

1872.000
MGAL
MCORR

$.290 \quad 979951.847$

$.300 \quad 979955.034$

$.190 \quad 979957.301$

$.370 \quad 979959.74 \mathrm{~B}$

$.210 \quad 979962.430$

$.200 \quad 979962.882$

$.320 \quad 979948.516$

$.110 \quad 979948.123$

$.170 \quad 979948.437$

$.410 \quad 979948$

$.370 \quad 979944.405$

30097929.473

$270 \quad 979950.062$

$.290 \quad 979943.619$

$310 \quad 979939.199$

$.310 \quad 979939.199$

.250 .979923 .792

$.280 \quad 979923.805$

$.280 \quad 979924.520$

.34097936 .869

.270 97934.227

$.330 \quad 979912.668$

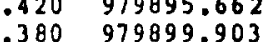

$.450 \quad 979899.895$

$.360 \quad 979919.488$

$.550 \quad 979919.535$

$.120 \quad 979913.345$

$.130 \quad 979910.326$

$.150 \quad 979912.854$

$.220 \quad 979919.279$

$.480 \quad 979915.031$

.370 979904.373

$.220 \quad 979897.110$

.170
$.260 \quad 979867.179$
PREE AIR

$-16.762$

$-19.072$

$-19.510$

$-20.513$

$-22.015$

$-20.753$

$-15.933$

$-13.225$

$-15.622$

$-12.480$

$-12.480$

$-17.427$

$-19.703$

$-15.799$

$-16.000$

$-4.319$

$-4.415$

$-16.533$

$-23.364$

$-4.222$

1.420

2.492

$-11.848$

$-12.019$

$-.142$

$-11.194$

$-11.624$

$-13.150$

10.912

$-8.921$

12.140

11.933
BOUGUER

MGAL

THEOR G

MGAL :

T C BOUGUER

MGAL

$-46.606 \quad 980050.912$

$-47.662900055 .1330-47.292$

$-47.293 \quad 900056.369-47.133$

$-48.346 \quad 980057.060 \quad-48.236$

-47.697 900057.943 -47.497

$-47.653 \quad 980051.927-47.333$

$-45.627900050 .706 \quad-45.517$

$-46.309000049 .604 \quad-46.139$

$-46.59190049 .604-46.139$

$-45.07100048 .972-45.501$

$-45.724 \quad 900049.001-45.584$

$-46.077900047 .016 \quad-45.687$

$-47.603900046 .7000-47.333$

$-47.280 \quad 980046.237-46.0990$

$-50.050 \quad 980045.605=-49.740$

$-47.617900045 .091-47.427$

$-46.47590004 .371 \quad-46.225$

$-46.5389044 .371-46.225$

$-47.279980043 .4450 .46 .258$

$-48.901980042 .6660-40.561$

$-47.47290042 .665-48.561$

$-48.562 \quad 900039.170 \quad-40.232$

$-50.764900030 .157-50.344$

$-47.703900036 .938 \quad-47.323$

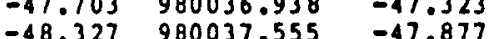

$-50.350 \quad 980036.967 \quad-49.790$

$-49.91390036 .057-49.790$

$-45.504 \quad 980038.348 \quad-45.363$

$-46.698 \quad 900038.062-45.568$

$\begin{array}{lll}-45.698 & -40038.862 & -46.568\end{array}$

$-49.394 \quad 900035.4822 \quad-45.377$

$-50.0160005 .822-49.174$

$-52.91670035 .014 \quad-50.436$

$-52.98790034 .588-52.567$

$-52.421 \quad 980034.148 \quad-52.031$

$-53.575 \quad 90033.693 \quad-52.208$

$-53.245 \quad 980033.091 \quad-53.025$

$\begin{array}{lll}-52.709 . & 980032.078 & -52.539 \\ -51.916 & 980031.330 & -51.656\end{array}$ 
PAINCIPAL PACTSAT GRAVITY BTATIONB

\begin{tabular}{|c|c|c|c|c|c|c|c|c|c|c|}
\hline TA NAME & AND NUM & $\begin{array}{c}\text { LATITUDE } \\
\text { NORTH + }\end{array}$ & $\begin{array}{c}\text { LONG ITUDE } \\
\text { EAST }+\end{array}$ & $\begin{array}{c}\text { ELEVATION } \\
\text { FEET }\end{array}$ & $\begin{array}{l}\text { ERR CORR } \\
\text { MGAL }\end{array}$ & $\begin{array}{l}\text { OBSFRVED } G \\
\text { MGAL }\end{array}$ & $\begin{array}{l}\text { PREE AIR } \\
\text { MGAL }\end{array}$ & $\begin{array}{c}\text { BOUGUER } \\
\text { MGAL }\end{array}$ & $\begin{array}{l}\text { THEOR G } \\
\text { MGAL }\end{array}$ & $\begin{array}{c}\text { T EOUGUEF } \\
\text { MGNL }\end{array}$ \\
\hline $\begin{array}{l}5.216 \\
5.214 \\
5.212 \\
5.299 \\
5.201 \\
5.411 \\
5.410 \\
5.409 \\
5.408 \\
5.412 \\
5.413 \\
5.414 \\
5.407 \\
5.406 \\
5.405 \\
5.404 \\
5.415 \\
5.416 \\
5.417 \\
5.418 \\
5.419 \\
5.420 \\
5.403 \\
5.489 \\
5.402 \\
5.401 \\
5.311 \\
5.310 \\
5.309 \\
5.308 \\
5.371 \\
5.307 \\
5.306 \\
5.305 \\
5.304 \\
5.303 \\
5.302 \\
5.301 \\
5.311 \\
5.312\end{array}$ & $\begin{array}{l}200 \\
201 \\
202 \\
203 \\
204 \\
205 \\
206 \\
207 \\
208 \\
209 \\
210 \\
211 \\
212 \\
213 \\
214 \\
215 \\
216 \\
217 \\
218 \\
219 \\
220 \\
221 \\
222 \\
223 \\
221 \\
225 \\
226 \\
227 \\
228 \\
229 \\
230 \\
231 \\
232 \\
233 \\
234 \\
235 \\
236 \\
237 \\
238 \\
239\end{array}$ & $\begin{array}{ll}38 & 26.77 \\
38 & 25.67 \\
38 & 25.07 \\
38 & 24.71 \\
38 & 22.58 \\
38 & 22.20 \\
38 & 22.03 \\
38 & 21.51 \\
38 & 21.15 \\
38 & 21.22 \\
38 & 21.50 \\
38 & 21.55 \\
38 & 20.78 \\
38 & 20.42 \\
38 & 19.80 \\
38 & 18.68 \\
38 & 18.76 \\
38 & 19.19 \\
38 & 19.33 \\
38 & 20.17 \\
38 & 18.84 \\
38 & 18.90 \\
38 & 17.55 \\
38 & 17.10 \\
38 & 16.87 \\
38 & 17.37 \\
38 & 18.33 \\
38 & 18.57 \\
38 & 18.33 \\
38 & 18.20 \\
38 & 18.20 \\
38 & 18.02 \\
38 & 17.25 \\
38 & 16.84 \\
38 & 17.89 \\
38 & 16.38 \\
38 & 16.40 \\
38 & 16.37 \\
38 & 17.86 \\
38 & 17.56\end{array}$ & 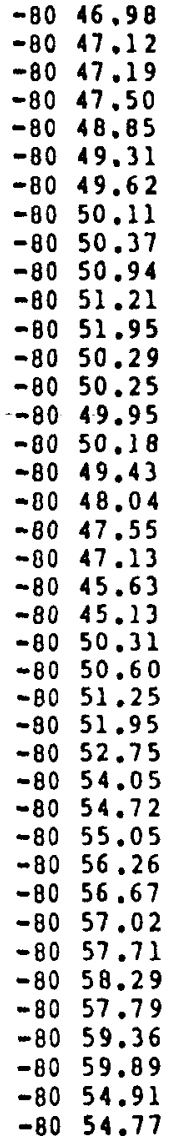 & $\begin{array}{l}1880.000 \\
2040.000 \\
1885.000 \\
1885.000 \\
1865.000 \\
1862.000 \\
1851.000 \\
1880.000 \\
1853.000 \\
1909.000 \\
1880.000 \\
1875.000 \\
1870.000 \\
1855.000 \\
1890.000 \\
1850.000 \\
1915.000 \\
1920.000 \\
1923.000 \\
2000.000 \\
2083.000 \\
2085.000 \\
1944.000 \\
1894.000 \\
1920.000 \\
1725.000 \\
1581.000 \\
1515.000 \\
1530.000 \\
1528.000 \\
1311.000 \\
1299.000 \\
1268.000 \\
1207.000 \\
1207.000 \\
1165.000 \\
1160.000 \\
1153.000 \\
1480.000 \\
1518.000\end{array}$ & $\begin{array}{l}.170 \\
.290 \\
.370 \\
.380 \\
.350 \\
.280 \\
.350 \\
.340 \\
.710 \\
.420 \\
.450 \\
.340 \\
.390 \\
.410 \\
.190 \\
.210 \\
.150 \\
.110 \\
.170 \\
.270 \\
.210 \\
.120 \\
.140 \\
.100 \\
.120 \\
.310 \\
.180 \\
.220 \\
.340 \\
.110 \\
.130 \\
.170 \\
.100 \\
.270 \\
.380 \\
.710 \\
.410 \\
.200 \\
.160 \\
.170\end{array}$ & $\begin{array}{l}979867.209 \\
979855.236 \\
979866.017 \\
979865.801 \\
979860.501 \\
979860.538 \\
979860.575 \\
979860.602 \\
979860.644 \\
979860.682 \\
979860.719 \\
979860.762 \\
979860.838 \\
979860.876 \\
979852.150 \\
979852.536 \\
979847.886 \\
979848.823 \\
979850.532 \\
979847.611 \\
979839.173 \\
979839.220 \\
979850.775 \\
979853.195 \\
979852.966 \\
979862.097 \\
979878.460 \\
979881.443 \\
979880.303 \\
979879.800 \\
979889.008 \\
979893.838 \\
979894.542 \\
979897.959 \\
979897.983 \\
979898.460 \\
979898.489 \\
979898.517 \\
979881.624 \\
979879.077\end{array}$ & $\begin{array}{l}12.730 \\
17.421 \\
14.503 \\
14.815 \\
10.759 \\
11.071 \\
10.323 \\
13.840 \\
11.871 \\
17.073 \\
13.972 \\
13.472 \\
14.206 \\
13.361 \\
8.836 \\
7.103 \\
8.449 \\
9.226 \\
11.012 \\
14.101 \\
15.421 \\
15.568 \\
15.840 \\
14.216 \\
16.769 \\
6.826 \\
8.237 \\
4.659 \\
5.283 \\
4.782 \\
-6.421 \\
-2.456 \\
-3.539 \\
-5.259 \\
-6.774 \\
-8.034 \\
-8.505 \\
-9.092 \\
2.590 \\
4.056\end{array}$ & $\begin{array}{l}-51.392 \\
-52.158 \\
-49.789 \\
-49.477 \\
-52.851 \\
-52.437 \\
-52.810 \\
-50.282 \\
-51.330 \\
-48.038 \\
-50.119 \\
-50.479 \\
-49.574 \\
-49.908 \\
-55.627 \\
-55.996 \\
-56.867 \\
-56.260 \\
-54.576 \\
-54.113 \\
-55.625 \\
-55.545 \\
-50.465 \\
-50.383 \\
-48.716 \\
-52.009 \\
-45.687 \\
-47.013 \\
-46.901 \\
-47.331 \\
-51.136 \\
-46.862 \\
-46.787 \\
-46.427 \\
-47.942 \\
-47.769 \\
-48.070 \\
-48.417 \\
-47.889 \\
-47.718\end{array}$ & $\begin{array}{l}980031.315 \\
980029.701 \\
980028.820 \\
980028.292 \\
980025.167 \\
980024.610 \\
980024.360 \\
980023.597 \\
980023.069 \\
980023.172 \\
980023.583 \\
980023.656 \\
980022.527 \\
980021.999 \\
980021.090 \\
980019.448 \\
980019.565 \\
980020.195 \\
980020.401 \\
980021.632 \\
980019.682 \\
980019.770 \\
980017.791 \\
980017.131 \\
980016.794 \\
980017.527 \\
980018.935 \\
980019.286 \\
980018.935 \\
980018.744 \\
980018.744 \\
980018.480 \\
980017.351 \\
980016.750 \\
980018.289 \\
980016.076 \\
980016.106 \\
980016.062 \\
980018.246 \\
980017.806\end{array}$ & $\begin{array}{l}-51.222 \\
-51.868 \\
-49.419 \\
-49.097 \\
-52.501 \\
-52.157 \\
-52.460 \\
-49.942 \\
-50.620 \\
-47.618 \\
-49.699 \\
-50.139 \\
-49.184 \\
-49.498 \\
-55.437 \\
-55.786 \\
-56.717 \\
-56.150 \\
-54.406 \\
-53.843 \\
-55.415 \\
-55.425 \\
-50.325 \\
-50.283 \\
-48.596 \\
-51.699 \\
-45.507 \\
-46.793 \\
-46.561 \\
-47.224 \\
-51.006 \\
-46.592 \\
-46.687 \\
-46.157 \\
-47.562 \\
-47.059 \\
-47.660 \\
-48.217 \\
-47.729 \\
-47.548\end{array}$ \\
\hline
\end{tabular}


PRINCIPAL PACTSATERAVITY BTATIONB

STA NAME AND NUM

$\begin{array}{ll}5.313 & \\ 5.314 & 240 \\ 5.315 & 241 \\ 5.316 & 242 \\ 5.317 & 243 \\ 5.101 & 244 \\ 5.103 & 245 \\ 5.104 & 246 \\ 5.105 & 247 \\ 5.101 & 248 \\ 5.107 & 249 \\ 5.108 & 250 \\ 5.109 & 251 \\ 5.110 & 252 \\ 5.111 & 253 \\ 5.112 & 254 \\ 5.113 & 255 \\ 5.114 & 256 \\ 5.171 & 257 \\ 5.116 & 258 \\ 5.117 & 259 \\ 5.172 & 260 \\ 5.115 & 261 \\ 8.314 & 262 \\ 8.322 & 263 \\ 8.323 & 264 \\ 8.324 & 265 \\ 8.321 & 266 \\ 8.320 & 267 \\ 8.319 & 268 \\ 8.318 & 269 \\ 5.229 & 270 \\ 5.230 & 271 \\ 5.120 & 272 \\ 5.119 & 273 \\ 5.118 & 275 \\ 5.228 & 276 \\ 5.227 & 277 \\ 5.226 & 278 \\ 5.225 & 279\end{array}$

LATITUDE
NORTH +

3816.96

$38 \quad 16.45$

$38 \quad 15.97$

3821.93

822.78

33.8
8

$\begin{array}{lll}38 & 23.93\end{array}$

$38 \quad 24.54$

$38 \quad 24.97$

$38 \quad 25.76$

3826.15

$38 \quad 26.59$

3826.93

$\begin{array}{lll}38 & 27.36 \\ 38 & 27.39\end{array}$

$\begin{array}{lll}8 & 27.36 \\ 38 & 27.43\end{array}$

$38 \quad 27.56$

$\begin{array}{lll}38 & 28.05\end{array}$

3828.00

$\begin{array}{ll}8 & 27.9 \\ 3 & 28.9\end{array}$

3830.50

$38 \quad 32.19$

3832.53

$\begin{array}{ll}38 & 33.08\end{array}$

$\begin{array}{lll}38 & 31.23 \\ 38 & 31.74\end{array}$

$38 \quad 31.38$

$38 \quad 31.38$

$38 \quad 28.62$

$\begin{array}{lll}38 & 28.59 \\ 8 & 28.29\end{array}$

$38 \quad 28.78$

B 29.66

3829.66

3829.3

3829.82

38
38 29.62
LONG ITUDE
EAST +

BLEVATION
FEET

TERR

MGAL

MGAL

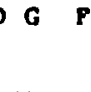

MGal

OUGUER
MGAL

THEOR G
MGAL

T C BOUGUER $\begin{array}{ll}-80 & 54.65 \\ -80 & 54.54\end{array}$

1525.000
1525.000

1890.000

1888.000

1952.000

1760.000

2779.000

1719.000

1725.000

1730.000

1383.000

1140.000

1000.000

943.000

840.000

842.000

828.000

828.000

880.000

84.000

826.000

1095.000

891.000

920.000

920.000

941.000

1101.000

1422.000

1646.000

1625.000

1625.000
1620.000

1210.000

1210.000
1180.000

1268.000

1268.000

1199.000

1730.000

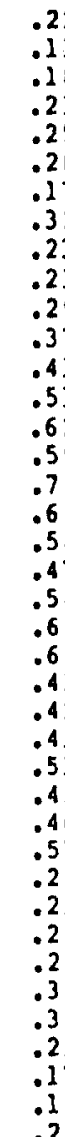

$.220 \quad 979878.351$

979874.867
.80

10 979074.906

.250 979863.632

$170 \quad 979874.455$

$.320 \quad 979871.633$

$230 \quad 979876.394$

$210 \quad 979876.137$

$290 \quad 979876.514$

$370 \quad 979898.575$

410979912.899

$510 \quad 979921.346$

$\begin{array}{ll}620 & 979925.027\end{array}$

$90 \quad 979933.037$

$720 \quad 979933.058$

979934.377

. 979933.322

54097934.089

$690 \quad 979934.173$

600979934.993

$410 \quad 979923.856$

4079070

.410 979937.403

510 979937.44

$450 \quad 979935.275$

$460 \quad 979925.274$

$570 \quad 979905.027$

$250 \quad 979905.056$

210979890.865

$.290 \quad 979890.889$

$270 \quad 979890.903$

310979914.775

$340 \quad 979916.180$

$210 \quad 979911.622$

079915.718

$190 \quad 979917.56$

$\begin{array}{ll}-80 & 49.40 \\ -80 & 47.82\end{array}$
.280 979863.27

5.338
2.132

2.132

17.195

16.557

21.419

13.032

11.821

10.043
9.720

10.112

$-1.171$

$-10.276$

$-15.643$

$-17.823$

-20.133
-19.968

$-20.023$

$-21.270$

$-16.331$

-19.99
-21.22

$-21.049$

-21.049
-9.937

$-17.669$

$-15.833$

$-14.077$

$-9.776$

9.776
.700

.700
.728

11.660

9.752

9.736
-5.676

$-8.385$

$-4.034$

$-6.575$

$-6.954$

-6.958
9.383
$-46.816 \quad 980016.926$

980298016.179

49.139980015 .475

$47.268 \quad 980024.213$

$-47.837980024 .698$

45.158980025 .460

$-46.997980026 .97$

$-48.855980027 .147$

$-48.587980028 .042$

$49.115 \quad 980028.673$

$-48.341 \quad 980029.833$

$-49.158 \quad 980030.405$

$-49.750 \quad 980031.051$

$-48.783980032 .181$

$-48.686 \quad 980032.225$

$-48.264980032 .284$

$-49.511980032 .474$

$-46.345 \quad 980033.194$

$48.646 \quad 980033.120$

$-49.396 \quad 980033.091$

$-49.222 \quad 980033.737$

$-47.285 \quad 980036.79$

$-48.058 \quad 980039.27$

47.211980039 .77

$-47.981 \quad 980040.580$

$-472980037.86$

980038.61

$-47.772980038 .083$

$-44: 481 \quad 980034.031$

$-45.672-980033.987$

$-45.518 \quad 980033.546$

$-46.946980034 .265$

$-48.631980035 .558$

$-47.282 \quad 980034.926$

$-47.470 \quad 980035.073$

$-47.303 \quad 980035.792$

980035.499
$-46.626$

$-49.772$

$-48.95$

$-47.058$

$-44.878$

$-46.827$

$-48.535$

$-48.357$

$-48.905$

$-48.603$

$-47.971$

$-48.748$

$-49.240$

$-49.366$

$-48.193$

$-47.574$

$-48.931$

$-45.87$

$-48.706$

$-48.542$

$-46.87$

47.638

$-46.801$

$-45.722$

$-46.868$

$-47.231$

$-47.522$

$-44.271$

$-45.248$

$-46.636$

$-48.291$

$-47.072$

$-47.300$

$-49.343$ 
PRINCIPAL PACISAT GRAVITY BTATIONB

STA NAME AND NUM

$\begin{array}{ll}11.301 & 280 \\ 11.302 & 281 \\ 11.303 & 282 \\ 11.304 & 283 \\ 11.305 & 284 \\ 11.306 & 285 \\ 11.307 & 286 \\ 11.308 & 287 \\ 11.309 & 288 \\ 11.313 & 289 \\ 11.318 & 290 \\ 11.315 & 291 \\ 13.417 & 717 \\ 11.316 & 292 \\ 11.317 & 293 \\ 11.318 & 294 \\ 11.319 & 295 \\ 11.320 & 296 \\ 11.321 & 297 \\ 11.322 & 298 \\ 11.323 & 299 \\ 11.310 & 300 \\ 11.311 & 301 \\ 11.312 & 302 \\ 11.101 & 303 \\ 11.101 & 304 \\ 11.103 & 305 \\ 11.104 & 306 \\ 11.105 & 307 \\ 11.106 & 308 \\ 11.107 & 309 \\ 11.108 & 310 \\ 11.199 & 311 \\ 11.109 & 312 \\ 11.110 & 313 \\ 11.111 & 314 \\ 11.112 & 315 \\ 11.113 & 316 \\ 11.114 & 317 \\ 11.115 & 318 \\ 11 & \\ & \end{array}$

LATITUDE

3839.10 3838.50

3838.52

3838.51

838.52

3838.7

3839.15

3.841 .20

38 12.07

3843.30

3831.18

38 44.11

3843.36

$\begin{array}{lll}38 & 43.30 \\ 38 & 43.30 \\ 38 & 43.6\end{array}$

$\begin{array}{ll}38 & 43.30 \\ 38 & 43.61\end{array}$

3844.96

$\begin{array}{lll}38 & 44.55\end{array}$

$\begin{array}{ll}38 & 44.32 \\ 38 & 50.76\end{array}$

$\begin{array}{ll}38 & 50.76 \\ 38 & 51.72\end{array}$

3851.72

$\begin{array}{ll}38 & 51.82 \\ 38 & 52.79\end{array}$

$\begin{array}{ll}38 & 52.79 \\ 38 & 53.11\end{array}$

853.36

3853.74

3854.53

3854.72

$38 \quad 55.19$

3855.29

3855.38

3856.40

3857.23

$38 \quad 57.75$

3858.16

3858.7

$38 \quad 59.37$

3856.00
LONG ITUDE

$\begin{array}{ll}-80 & 45.41\end{array}$ $-8045.73$ $\begin{array}{rr}-80 & 45.73 \\ -80 & 45.73\end{array}$ $\begin{array}{rr}-80 & 45.74\end{array}$ $\begin{array}{ll}-80 & 47.57 \\ -80 & 47.97\end{array}$ -80 47.97 $-80 \quad 49.87$ $-80 \quad 49.08$ $-8049.66$ $\begin{array}{lll}-80 & 49.95\end{array}$ $\begin{array}{ll}-80 & 49.73\end{array}$ $\begin{array}{ll}-80 & 49.27\end{array}$ 48.57 $\begin{array}{lll}-80 & 48.15\end{array}$ $-80 \quad 47.53$ $\begin{array}{rrr}-80 & 46.74\end{array}$ $\begin{array}{lll}-80 & 46.16\end{array}$ $\begin{array}{lll}-80 & 45.33\end{array}$ $\begin{array}{ll}-80 & 40.13 \\ -80 & 39.90\end{array}$ -80 11.95 $\begin{array}{lll}-80 & 43.02\end{array}$ $\begin{array}{ll}-80 & 43.23\end{array}$ $\begin{array}{lll}-80 & 43.62\end{array}$ $-80 \quad 43.89$ $-80 \quad 44.00$ 44.11
0

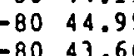
$\begin{array}{lll}-80 & 43.66\end{array}$ $\begin{array}{lll}-80 & 43.20\end{array}$ $-8043.89$ -80
-80 43.8

$\begin{array}{ll}-80 & 43.3 \\ -80 & 43.8\end{array}$

$\begin{array}{lll}-80 & 43.76\end{array}$

-80
-80
42.80

\section{EVVATION}

891.000

07.000

873.000

871.000

874.000

870.000

875.000

075.000

840.000

838.000

1126.000

871.000

615.000

929.000

920.000

1980.000

1028.000

980.000

871.000

923.000

760.000

780.000

802.000

756.000

753.000

755.000

750.000

748.000

745.000

775.000

747.000

750.000

740.000

800.000

812.000

880.000

1118.000

1118.000

760.000 cor cora MGAL MGAL

MGAL

BOUGUER

THEOR G

C BOUGUER

\section{$.150 \quad 979948.136$}

.17097950 .070

979950.252

$-17.183$

$-16.475$

$-16.577$

$-16.279$

$-16.670$

.

$-15.059$

-15.474

$-15.645$

$-15.71$

$-34.238$

$-14.781$

$-16.708$

$-14.985$

$-9.810$

$-14.922$

$-12.838$

$-16.974$

$-14.678$

$-19.245$

$-17.925$

$-16.470$

$-18.562$

$-18.102$

$-18.578$

$-18.808$

$-19.059$

$-18.612$

$-17.739$

$-18.227$

$-18.065$

$-19.183$

$-17.806$

$-17.249$

$-14.581$

$-5.394$

-6.331
-18.036

\section{-47.872
-46.135}

980049.427

98048.560

90048.575

980048.575

980048.854

980049.501

980052.515

980052.500

980053.795

900037.60

90037.79

980056.795

980055.692

90055.604

90055.604

98056.060

90058.046

90057.143

980066.583

980067.997

90006.572

980070.044

980070.412

90070.972

90072.135

900072.415

900073.100

980073.255

980073.388

980074.892

90076.114

9007604

980077.484

$-43.526 \quad 980078.324$

$-44.463980079 .268$

$-43.958 \quad 980074.301$
$-47.722$

$-45.965$

4.868

45.97

$-46.153$

. 06

$-48.763$

$-43.854$

$-4.917$

$-45.052$

$-54.824$

$-46.066$

$-47.677$

4.940

$-19.664$

$-45.843$

$-46.172$

$-44.897$

$-44.279$

$-43.494$

$-43.275$

$-43.759$

$-43.769$

4.152

$-43.862$

$-43.415$

$-43.645$

$-44.082$

$-44.505$

$-44.105$

$-43.136$

$-43.688$ 
PRINCI PAL PACTENATGRAVITY BTATIONS

STA NAKE AND NUM

$\begin{array}{ll}11.118 & 319 \\ 11.119 & 320 \\ 11.120 & 321 \\ 11.121 & 322 \\ 11.122 & 323 \\ 11.201 & 324 \\ 11.202 & 325 \\ 11.203 & 326 \\ 11.204 & 327 \\ 11.205 & 328 \\ 11.206 & 329 \\ 11.207 & 330 \\ 11.208 & 331 \\ 11.209 & 332 \\ 11.210 & 333 \\ 11.211 & 334 \\ 11.212 & 335 \\ 11.213 & 336 \\ 11.214 & 337 \\ 11.175 & 338 \\ 11.176 & 339 \\ 11.177 & 340 \\ 11.117 & 341 \\ 11.116 & 342 \\ 11.422 & 343 \\ 11.421 & 344 \\ 11.420 & 345 \\ 11.419 & 346 \\ 11.418 & 347 \\ 11.417 & 348 \\ 11.416 & 349 \\ 11.415 & 350 \\ 11.410 & 351 \\ 11.411 & 352 \\ 11.412 & 353 \\ 11.413 & 354 \\ 11.409 & 355 \\ 11.408 & 356 \\ 11.405 & 357 \\ 11.403 & 358 \\ 11 & \\ & 319 \\ 1\end{array}$

LAT ITUDE
HORTH +

3856.67

$38 \quad 57.10$

3857.61

3858.18

3858.43

38 57.14

$38 \quad 57.91$

$38 \quad 58.03$

$38 \quad 57.75$

3857.09

855.85

$38 \quad 56.20$

3855.63

$\begin{array}{ll}38 & 56.03 \\ 38 & 56.14\end{array}$

3855.55

3855.89

3855.60

3855.82

3855.50

3855.55

3855.78

3851.55

3851.83

3852.05

$\begin{array}{ll}38 & 51.67\end{array}$

$\begin{array}{ll}38 & 51.36\end{array}$

3851.39

3851.02

3848.03

$\begin{array}{lll}38 & 48.036 \\ 38 & 49.00\end{array}$

3848.60

3847.19

3848.89

3847.03

38
38 47.47
LONG I TUDE
LAST +

$\begin{array}{rr}-80 & 41.15 \\ -80 & 40.40\end{array}$

$\begin{array}{rr}-80 & 40.40 \\ -80 & 40.29\end{array}$

-80
-80.29
-8038.89

$\begin{array}{ll}-80 & 37.91\end{array}$

$-8036.68$

$-8033.56$

$\begin{array}{ll}-80 & 32.40\end{array}$

$\begin{array}{lll}-80 & 30.74\end{array}$

-8030.95
-80

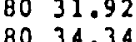

$\begin{array}{lll}-80 & 33.31\end{array}$

$\begin{array}{ll}-80 & 34.20 \\ -80 & 34.91\end{array}$

$-80 \quad 35.68$

$\begin{array}{ll}-80 & 36.25\end{array}$

$\begin{array}{lll}-80 & 37.43\end{array}$

$\begin{array}{ll}-80 & 38.51 \\ -80 & 39.85\end{array}$

$\begin{array}{ll}-80 & 39.85 \\ -80 & 40.653\end{array}$

$\begin{array}{lll}-80 & 41.51\end{array}$

$\begin{array}{lll}-80 & 41 .\end{array}$

$\begin{array}{lll}-80 & 39.92\end{array}$

$\begin{array}{ll}-80 & 34.35\end{array}$

$\begin{array}{ll}-80 & 33.39\end{array}$

$\begin{array}{lll}-80 & 33.07\end{array}$

$\begin{array}{lll}-80 & 32.01\end{array}$

$\begin{array}{ll}-80 & 31.29\end{array}$

$\begin{array}{rl}-80 & 30.45\end{array}$

$\begin{array}{ll}-80 & 30.18 \\ -80 & 30.00\end{array}$

-80
31.00

$\begin{array}{lll}-80 & 30.82\end{array}$

$\begin{array}{lll}-80 & 31.77\end{array}$

$\begin{array}{ll}-80 & 31.15\end{array}$

$\begin{array}{lll}-80 & 31.86\end{array}$

$\begin{array}{ll}-80 & 33.90 \\ -80 & 35.39\end{array}$
ELEVATION
FEET

\section{N}

TERR CORR

MGAL

MCIAL
MERVPO

FREE AIR
MGNL

$-17.992$

762.000
810.000

810.000

812.000
792.000

825.000

868.000

855.000

884.000

940.000

1300.000

1240.000

1231.000

1020.000

1020.000

921.000

890.000

871.000

851.000

815.000

815.000

763.000

863.000

850.000

755.000

801.000

820.000

826.000

853.000
941.000

1015.000

1015.000

1373.000

1020.000

940.000

980.000

830.000

1050.000

791.000

791.000

820.000

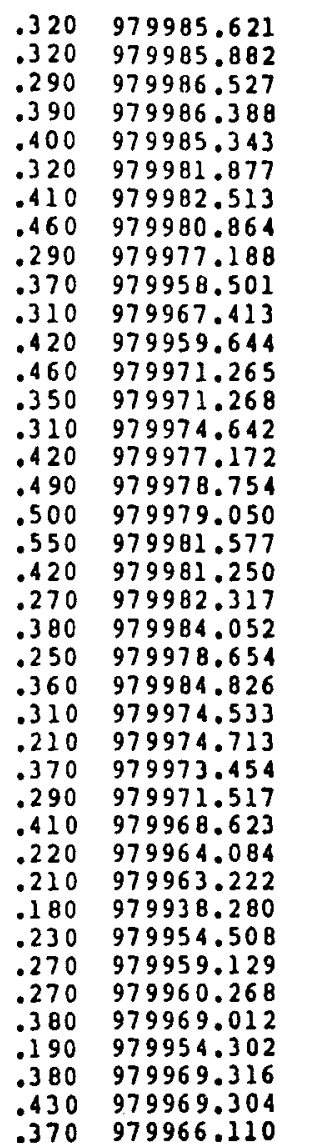

$-17.992$ $-13.768$ $-16.629$ $-14.939$ -12.459
-13.207 $-13.207$ -13.102
-11.687 3.901 $-1.265$ $-.075$ $-6.872$ $-7.386$ $-12.483$ $-13.458$ $-13.826$ $-14.542$ $-15.055$ $-15.530$ $-17.744$ $-15.032$ $-18.135$ $-17.870$ $-16.315$ -16.315
-17.333 $-16.171$ $-10.331$ $-6,513$ $-8.346$ .461 $-12.114$ $-15.503$ $-11.543$ $-8.321$ $-20.111$ $-17.385$

\begin{tabular}{|c|c|c|}
\hline $\begin{array}{l}\text { OUGUER } \\
\text { MGAL }\end{array}$ & $\begin{array}{c}\text { THEOR G } \\
\text { MGAL }\end{array}$ & $\begin{array}{l}\text { T. C BOUGUER } \\
\text { MGAL }\end{array}$ \\
\hline $\begin{array}{l}-43.982 \\
-41.477 \\
-41.463 \\
-43.642 \\
-43.077 \\
-42.064 \\
-42.369 \\
-43.252 \\
-43.747 \\
-40.439 \\
-40.147 \\
-42.061 \\
-41.661 \\
-42.175 \\
-43.896 \\
-43.814 \\
-43.533 \\
-43.567 \\
-43.159 \\
-43.599 \\
-43.156 \\
-43.767 \\
-44.023 \\
-43.886 \\
-45.190 \\
-44.283 \\
-45.506 \\
-45.265 \\
-42.426 \\
-43.132 \\
-43.135 \\
-46.368 \\
-46.903 \\
-47.564 \\
-44.968 \\
-44.629 \\
-44.074 \\
-47.090 \\
-44.364 \\
-46.467\end{array}$ & $\begin{array}{l}980075.288 \\
980075.922 \\
980076.674 \\
980077.514 \\
980077.882 \\
980075.981 \\
980076.143 \\
980077.116 \\
980077.293 \\
980076.880 \\
980075.907 \\
980075.509 \\
980074.080 \\
980074.596 \\
980073.756 \\
980074.345 \\
980074.507 \\
980073.638 \\
980074.139 \\
980073.712 \\
980074.036 \\
980073.564 \\
980073.638 \\
980073.977 \\
980067.746 \\
980068.159 \\
980068.483 \\
980067.923 \\
980067.466 \\
980068.070 \\
980067.511 \\
980066.966 \\
980062.564 \\
980063.050 \\
980063.992 \\
980063.103 \\
980061.328 \\
980063.830 \\
980061.092 \\
980061.740\end{array}$ & $\begin{array}{l}-43.662 \\
-41.157 \\
-41.173 \\
-43.252 \\
-42.677 \\
-41.744 \\
-41.959 \\
-42.792 \\
-43.457 \\
-40.069 \\
-39.837 \\
-41.641 \\
-41.201 \\
-41.825 \\
-43.586 \\
-43.394 \\
-43.043 \\
-43.067 \\
-42.609 \\
-43.179 \\
-42.886 \\
-43.387 \\
-43.773 \\
-43.526 \\
-44.880 \\
-44.073 \\
-45.136 \\
-44.975 \\
-42.016 \\
-42.912 \\
-42.925 \\
-46.188 \\
-46.673 \\
-47.294 \\
-44.698 \\
-44.249 \\
-43.884 \\
-46.710 \\
-43.934 \\
-46.097\end{array}$ \\
\hline
\end{tabular}


PRINCIPAL PACTEATEGRAVITY BTATIONE

STA NAHE AND NUM

$\begin{array}{rr}11.402 & 359 \\ 11.401 & 360 \\ 22.220 & 361 \\ 22.220 & 1078 \\ 4.420 & 362 \\ 4.419 & 363 \\ 4.418 & 364 \\ 4.417 & 365 \\ 4.416 & 366 \\ 4.415 & 367 \\ 4.414 & 368 \\ 4.412 & 369 \\ 4.411 & 370 \\ 4.410 & 371 \\ 4.409 & 372 \\ 2.202 & 373 \\ 2.201 & 374 \\ 2.122 & 375 \\ 2.212 & 376 \\ 2.120 & 377 \\ 2.119 & 378 \\ 2.116 & 379 \\ 2.117 & 380 \\ 2.118 & 381 \\ 4.301 & 382 \\ 4.333 & 383 \\ 4.334 & 384 \\ 4.335 & 385 \\ 4.336 & 386 \\ 4.337 & 387 \\ 4.303 & 388 \\ 4.302 & 389 \\ 4.399 & 390 \\ 4.304 & 391 \\ 4.305 & 392 \\ 4.306 & 393 \\ 4.307 & 394 \\ 4.308 & 395 \\ 4.309 & 396 \\ 4.310 & 397\end{array}$

LATITUDB NORTH +

3846.90 3846.14 $38 \quad 16.8$ 38 16.89 38 16.05 38 15.95 38.15 .50 38 15.37 $38 \quad 25.49$ 38 38. 15.32 $38 \begin{array}{ll}35.87 \\ 38\end{array}$ 3815.7 3814.92 3814.73 3814.15 3814.22 3813.62 3813.96 3813.72 3814.2 3814.79 3815.08 3815.07 $\begin{array}{lll}38 & 15.30 \\ 38 & 15.31\end{array}$ $\begin{array}{lll}38 & 15.71\end{array}$ 3815.78 3816.15 3815.56 38.72 815.89 3816.14 $38 \quad 16.38$ 3816.78 $38 \quad 17.02$ $\begin{array}{ll}38 & 17.35 \\ 38 & 17.34\end{array}$

\section{ONG ITUDE}

$-8036.00$ $-8036.80$ $-8051.49$ $\begin{array}{ll}-80 & 51.49\end{array}$ $-81 \quad .27$ $-81 \quad .49$ $-81 \quad 1.15$ $-811.70$ $\begin{array}{ll}-81 & 2.14\end{array}$ $-81 \quad 4.68$ -81 5.25 $\begin{array}{ll}-81 & 5.95\end{array}$ $\begin{array}{ll}-81 & 6.11 \\ -81 & 6.21\end{array}$ $\begin{array}{ll}-81 & 6.215\end{array}$ $-817.85$ $\begin{array}{ll}-81 & 8.27\end{array}$ -81 9.35 -81 11.28 - 1111.56 -8) 11.39 $-8111.52$ - 11.52 $-8113.32$ $\begin{array}{ll}-81 & 13.25\end{array}$ $\begin{array}{ll}-81 & 13.72\end{array}$ $-8111.71$ -81 11.81 $\begin{array}{lll}-81 & 11.79 \\ -81 & 11.07\end{array}$ $-8111.94$ $-8111.84$ $-8111.62$ $-8111.40$ $\begin{array}{ll}-81 & 11.68 \\ -81 & 11.67\end{array}$

\section{FEET}

870.000 850.000 1870.000 1870.000 1225.000 220.000 225.000 9.000 1200.000 1220.000 134.000 1324.000 1324.000 1299.000 80000 80.000 730.000 730.000 709.000 702.000 710.000 709.000 702.000 732.000 840.000 801.000 810.000 833.000 775.000 775.000 775.000 775.000
775.000 880.000 830.000 876.000 900.000 961.000 960.000

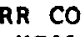

$-51.700 \quad 980017.498$
$-51.742 \quad 980017.483$

\section{$-45.577$}

$-43.173$

$-53.939$

$-50.294$

$-50.125$

$-50.524$

$-51.265$

$-49.753$

$-53.949$

$-54.462$

$-55.090$

$-53.952$

$-54.081$

$-53.935$

.54 .005

$-54.060$

$-53.592$

$-53.554$

$-53.382$

$-48.751$

$-53.226$

$-52.021$

$-47.314$

$-53.987$

$-54.004$

$-50.028$

$-52.819$

$-52.404$

$-51.230$

$-51.262$ 
PRINCI PAL PACTS AT GRAVITY BTATION

STA NAME AND NUM

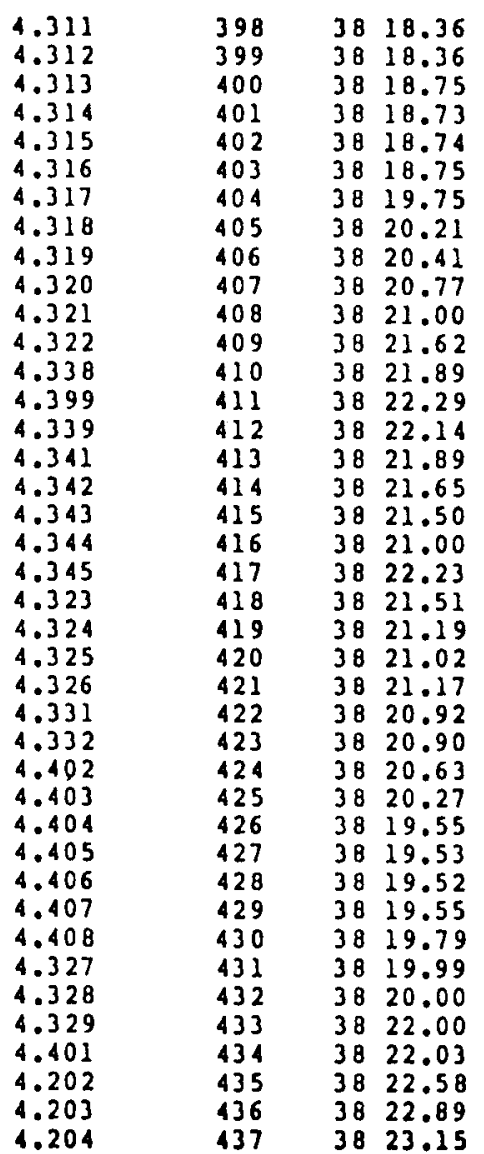

LONG ITUDE
EAST +

$-8111.17$ $\begin{array}{ll}-81 & 10.79\end{array}$ $-8110.52$ $\begin{array}{rl}-81 & 10.28 \\ -81 & 10.50\end{array}$ $\begin{array}{ll}-81 & 10.50 \\ -81 & 10.56\end{array}$ $\begin{array}{rll}-81 & 10.66 \\ -81 & 10.66\end{array}$ $-8110.21$ $-8110.51$ -81 9.80 $\begin{array}{ll}-81 & 9.71\end{array}$ $\begin{array}{ll}-81 & 9.51 \\ -81 & 9.70\end{array}$ $\begin{array}{ll}-81 & 9.70 \\ -81 & 10.10\end{array}$ $\begin{array}{ll}-81 & 10.10 \\ -81 & 10.35\end{array}$ $\begin{array}{ll}-81 & 10.35 \\ -81 & 10.51\end{array}$ $\begin{array}{ll}-81 & 10.51 \\ -81 & 10.75\end{array}$ -81 11.79 $-8111.50$ $\begin{array}{ll}-81 & 11.75 \\ -81 & 9.22\end{array}$ $\begin{array}{ll}-81 & 9.22\end{array}$ $\begin{array}{ll}-81 & 8.82 \\ -81 & 8.46\end{array}$ $\begin{array}{ll}-81 & 8.46 \\ -81 & 0.21\end{array}$ $\begin{array}{ll}-81 & 0.21 \\ -81 & 8.07\end{array}$ $\begin{array}{ll}-81 & 8.07 \\ -81 & 7.62\end{array}$ $\begin{array}{ll}-81 & 7.62 \\ -81 & 7.12\end{array}$ $-817.03$ $\begin{array}{ll}-81 & 6.97\end{array}$ $\begin{array}{ll}-81 & 6.49 \\ -81 & 5.93\end{array}$ $\begin{array}{ll}-81 & 5.93 \\ -81 & 5.51\end{array}$ $\begin{array}{ll}-81 & 5.51\end{array}$ $\begin{array}{ll}-81 & 7.68\end{array}$ $\begin{array}{ll}-81 & 7.55\end{array}$ $\begin{array}{ll}-81 & 7.59\end{array}$ $-81 \quad 7.48$ $\begin{array}{ll}-81 & 6.98\end{array}$ $\begin{array}{ll}-81 & 6.73 \\ -81 & 6.89\end{array}$

\section{ELATION
FEET}

1260.000 1258.000 1371.000 1371.000 1371.000 1371.000 1080.000 960.000 950.000 925.000 925.000 857.000 1080.000 806.000 860.000 880.000 875.000 880.000 840.000 1050.000 880.000 930.000 935.000 1043.000 980.000
987.000 1020.000 1060.000 1300.000 1300.000 1300.000 1300.000 1320.000 1278.000 1280.000 1170.000 1180.000 920.000 947.000
ERR CORR
MGAL MGAL PRER. MNL 80 .390
.380 $.380 \quad 979894.213$ 979894.216 .260979888 .248 .250 . $.290 \quad 979888.259$ $.350 \quad 979909.526$ $.390 \quad 979915.393$ $0.000 \quad 979916.464$

$.420 \quad 979918.409$

$.450 \quad 979918.983$

$.550 \quad 979923.294$

$.210 \quad 979914.307$

$.420 \quad 979928.089$

$.330 \quad 979926.317$

$.590 \quad 979924.558$

$0.000 \quad 979921.979$

$.550 \quad 979922.753$

$0.000 \quad 979921.717$

$.250 \quad 979916.338$

$.350 \quad 979925.652$

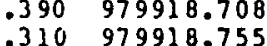

$.410 \quad 979911.353$

$.330 \quad 979910.732$

$.360 \quad 979910.736$

$.420 \quad 979908.927$

$.440 \quad 979906.123$

$.450 \quad 979892.650$

$.390 \quad 979892.652$

$.370 \quad 979892.654$

$.400 \quad 979892.656$

$.150 \quad 979889.898$ .090979889 .898 $.210 \quad 979905.543$ $.210 \quad 979905.542$ $.290 \quad 979924.658$ $.330 \quad 979920.932$

\section{$-6.248$} $-6.433$ $-2.305$ $-2.324$ $-2.333$ $-9.904$ $-15.999$ $-16.859$ $-19.854$ $-8.26$ $-20.839$ $-17.312$ $-16.823$ $-19.520$ $-18.055$ $-22.121$ $-15.17$ $-16.943$ $-16.176$ $-13.640$ $-19.128$ $-17.437$ -15.951
-5.793 $-5.793$ $-5.745$ $-5.788$ $-7.020$ $-11.260$ $-11.086$ $-7.825$ $-13.973$ $-11.800$

OUGUER
HGAL
TIIEOR
MGAL

T C BCUGUER MGNL

-49.223
-19.340

980018.978 980018.978 980019.550 $\begin{array}{ll}-49.066 & 980019.521 \\ -49.085 & 980019.536\end{array}$ $-49.094980019 .550$ $-46.740 \quad 980021.016$ $-48.742980021 .691$ $-48.563980021 .984$ $-48.646 \quad 980022.512$ $-48.408 \quad 980022.849$ $-49.084 \quad 980023.759$
$-45097 \quad 980024.155$ $-45.097980024 .155$ $-48.330 \quad 980024.742$ $-46.644980024 .521$ $-46.837980024 .155$ $-49.364980023 .803$ $-48.070 \quad 980023.583$ $-50.771 \quad 980022.849$ $-45.364980024 .654$ $-45.186 \quad 980023.597$ $-48.663 \quad 980023.128$ $-48.066 \quad 980022.879$ $-49.213980023 .099$ $-53.245980022 .732$ $-52.792980022 .703$ $-52.226 \quad 980022.307$ $-52.105 \quad 980021.779$ $-50.133 \quad 980020.723$ $-50.102980020 .694$ $-50.005 \quad 980020.679$
-50.127 .127980020 .723 $-52.041980021 .075$

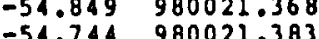
$-54.744980021 .383$ $-48.072900024 .360$ $-45.351980025 .167$ $-47.913 \quad 980025.622$ $-44.884980026 .003$
$-48.833$ $-48.960$ $-48.845$ $-48.776$ $-48.685$ $-48.004$ $-46.390$ $-48.352$ $-48.563$ $-48.226$ $-47.958$ $-48.534$ $-44.887$ $-47.910$ $-46.314$ $-46.247$ $-49.364$ $-47.520$ $-50.771$ -45.114
-44.836 $-48.273$ $-47.756$ $-48.803$ $-52.915$ $-52.432$ $-51.806$ $-51.665$ $-49.683$ $-49.712$ -49.715
-49.727 $-51.661$ $-54.699$ $-54.554$ $-48.417$ $-47.862$ $-45.061$ $-44.534$ 
PRINCI PAL PACTS.ATCA AVITY STATIONB

STA NAME AND NUM

$\begin{array}{ll}4.205 & 438 \\ 4.255 & 439 \\ 4.206 & 440 \\ 4.207 & 441 \\ 4.208 & 442 \\ 4.209 & 443 \\ 4.210 & 444 \\ 4.211 & 445 \\ 4.212 & 446 \\ 4.213 & 447 \\ 4.214 & 448 \\ 4.215 & 449 \\ 4.216 & 450 \\ 4.218 & 451 \\ 4.232 & 452 \\ 4.233 & 453 \\ 4.234 & 454 \\ 4.235 & 455 \\ 4.219 & 456 \\ 4.220 & 457 \\ 4.221 & 458 \\ 4.222 & 459 \\ 4.223 & 460 \\ 4.224 & 461 \\ 4.225 & 462 \\ 4.226 & 463 \\ 4.227 & 464 \\ 4.228 & 465 \\ 4.229 & 466 \\ 4.230 & 467 \\ 4.231 & 468 \\ 4.101 & 469 \\ 4.102 & 470 \\ 4.103 & 471 \\ 4.104 & 472 \\ 4.106 & 473 \\ 4.107 & 47 \\ 4.108 & 475 \\ 4.109 & 476 \\ 4.110 & 477 \\ & \end{array}$

LATITUDE
NORTH +

38 B 23.36

3823.75

$38 \quad 24.17$

38 24.37 3824.55

3824.78

3825.24

3825.58

3825.58

3825.83

$\begin{array}{ll}38 & 26.42\end{array}$

3826.80

38 27.55

38 26.82

$\begin{array}{lll}38 & 25.56\end{array}$

3824.84

3827.9

3828.53

$\begin{array}{ll}38 & 28.97\end{array}$

$38 \quad 29.55$

3829.39

$38 \quad 29.95$

3828.69

3829.17

$\begin{array}{ll}38 & 29.16\end{array}$

$\begin{array}{ll}38 & 29.19\end{array}$

3829.32

3829.11

3829.00

3829.52

38.96

$\begin{array}{lll}38 & 29.96 \\ 38 & 29.72\end{array}$

3829.48

LONGITUDE
EAST +

LEVATIO

FEET

900.000

877.000

877.000

877.000

800.000

797.000

797.000

730.000

726.000

20.000

701.000

730.000

712.000

712.000

715.000

1263.000

1263.000

1263.000

820.000

720.000

720.000

730.000

722.000

720.000

720.000

760.000

765.000

826.000

950.000

1032.000

946.000

910.000

840.000

840.000
780.000

760.000

760.0000

788.000

960.000

800.000
664.000
MER CORR
MGAL

MGAL

PREE AIR
MGAL

OUGUER

THEOR G

AGAL

T C BOUGUER

MGAL

$.410 \quad 979923.961$

$-17.694$

$-18.391980026 .311$

$-47.788 \quad 980026.88$

$-45.498 \quad 980026.252$

$-46.074980027 .499$

$-47.667980028 .057$

$-50.400 \quad 980028.395$

$-47.055980029 .070$

$-46.47908002968$

$-46.475$

-46.259 900029.994

$-46.360900030 .001$

$-44.981900031 .359$

$-45.556 \quad 980032.460$

$-45.84298003$

-46.012 980031.308

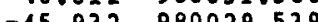

$-4.032580028 .583$

-43.541 900033.091

$-43.54100030 .091$

$-44.5309003000$

$-44.969980034$

$-46.59498003$

$-46.594900035030$

$-45.722980035 .939$

$-44.501980035 .983$

$-43.936 \quad 98003$

$-44.384980034 .133$

$-45.051980034 .691$

$-42.012980034 .838$

$-43.402 \quad 980034.823$
-42.548

$-12.548 \quad 980034.867$

$-44.643 \quad 980035.058$

$-45.446 \quad 980034.750$

$-41.650 \quad 980034.588$

$-44.432980035 .352$

$-43.797 \quad 980035.866$

$-42.791 \quad 980035.998$

$\begin{array}{ll}-45.159 & 980035.646 \\ -45.824 & 980035.293\end{array}$
$-47.981$

$-47.288$

-44.928
-46.324

$-47.827$

$-17.077$

$-49.780$

47.235

$-45.809$

$-47.865$

$-45.579$

$-45.658$

$-44.301$

$-45.036$

$-45.272$

$-46.182$

$-45.222$

$-44.165$

$-4.731$

$-43.948$

$-44.259$

$-46.074$

$-45.432$

$-45.162$

$-43.216$

$-43.894$

$-44.541$

$-42.972$

$-42.058$

$-44.223$

$-44.100$

$-43.862$

$-43.387$

$-42.301$

$-44.599$ 
PRINCI PA

C T $s$

GRAVITY

$\mathbf{s}$

LATITUDE

LONG ITUDE

TERR CORR

MGAL

$$
\text { MGNL }
$$

- prea air

4.115
4.116

$\begin{array}{lllll}478 & 38 & 29.05 & -81 & 12.26 \\ 479 & 38 & 28.54 & -81 & 12.35 \\ 480 & 38 & 28.52 & -81 & 11.81 \\ 481 & 38 & 28.67 & -81 & 11.46 \\ 482 & 38 & 28.16 & -81 & 11.30 \\ 483 & 38 & 28.01 & -81 & 11.32 \\ 484 & 38 & 27.54 & -81 & 11.65 \\ 485 & 38 & 26.96 & -81 & 11.13 \\ 486 & 38 & 26.77 & -81 & 11.70 \\ 487 & 38 & 26.04 & -81 & 11.95 \\ 488 & 38 & 26.16 & -81 & 11.12 \\ 489 & 38 & 25.68 & -81 & 10.96 \\ 490 & 38 & 26.25 & -81 & 10.39 \\ 491 & 38 & 26.85 & -81 & 10.48 \\ 492 & 38 & 27.10 & -81 & 10.12 \\ 493 & 3826.95 & -81 & 9.82 \\ 494 & 38 & 27.13 & -81 & 9.22 \\ 495 & 38 & 26.96 & -81 & 8.65 \\ 496 & 38 & 26.56 & -81 & 8.53 \\ 497 & 38 & 26.27 & -81 & 7.55\end{array}$

770.000

$.430 \quad 979943.336$

667.000

680.000

$590 \quad 979944.931$

979945.722

979947.058

660.000

659.000

4.119

4.120

4.121

4.122

4.123

4.124

4.126

4.127

4.128

4.130

4.131

4.132

4.133

4.134

497

700.000

699.000

665.000

670.000

675.000

680.000

680.000

670.000

670.000

690.000

680.000

680.000

690.000
680.000

$.630 \quad 979945.392$

.610979945 .472

979944.805

979943.086

979943.425
979941.483

979941.483
979941.444

979940.874

979941.293

979942.743

979942.286

979943.139

979942.703

$.890 \quad 979942.62$

.720
.979941 .204
979941.377

MGAL

BOUGUER
MGAL

THEOR G

MGAL

T C BOUGUER

$-18.898$

$-26.243$

$-45.162980034 .662$

$-21.203$

$-48.993 \quad 980033.913$

$-44.731$

$-46.772$

-45.078
-47.094

$-45.194 \quad 90033.355 \quad-46.564$
-25.077

$-25.677-48.153 \quad 980033.135 \quad-47.543$

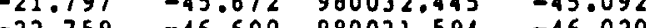

$-25.759-46.600 \quad 980031.594 \quad-46.020$

$-25.340-48.020190031 .315 \quad-47.310$

$-25.740-48.591 \quad 980030.244 \quad-47.841$

$-24.879-48.507 \quad 980030.420-47.787$

$-25.879-48.072 \quad 90029.7150-47.382$

$-25.668-48.590000 .532-47.810$

$-26.668-48.520 \quad 980031.432-47.800$

$-26.492-49.344 \quad 980031.799-48.454$

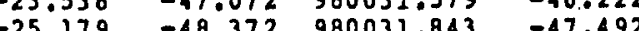

$-25.004 \quad-48.396 \quad 980031.594 \quad-47.492$

$\begin{array}{llll}-25.004 & -48.196 & 980031.594 & -47.306\end{array}$

$\begin{array}{llll}-24.900 & -48.431 & 980031.007 & -47.714 \\ -25.242 & -48.435 & 980030.581 & -47.535\end{array}$ 
PRINCIPAL PACTEAT GRAVITY GTATIONB

GTA NAME AND NUM

$\begin{array}{rr}2.116 & 1077 \\ 4.141 & 198 \\ 4.140 & 499 \\ 4.139 & 500 \\ 4.138 & 501 \\ 4.137 & 502 \\ 4.136 & 503 \\ 4.312 & 504 \\ 4.113 & 505 \\ 4.114 & 506 \\ 4.111 & 507 \\ 2.115 & 508 \\ 2.114 & 509 \\ 2.113 & 510 \\ 2.112 & 511 \\ 2.107 & 512 \\ 2.108 & 513 \\ 2.109 & 514 \\ 2.110 & 515 \\ 2.111 & 516 \\ 2.310 & 517 \\ 2.305 & 518 \\ 2.302 & 519 \\ 2.303 & 520 \\ 2.106 & 521 \\ 2.105 & 522 \\ 2.104 & 523 \\ 2.103 & 524 \\ 2.102 & 525 \\ 2.301 & 526 \\ 2.101 & 527 \\ 1.210 & 528 \\ 1.210 & 1076 \\ 1.208 & 529 \\ 1.207 & 530 \\ 1.211 & 531 \\ 1.212 & 532 \\ 1.213 & 533 \\ 1.214 & 534 \\ 1.215 & 535\end{array}$

\section{LATITUDE}

$38 \quad 13.72$

3823.63

38 23.63

$\begin{array}{ll}38 & 26.03\end{array}$

$\begin{array}{lll}38 & 27.07\end{array}$

38 29.52

3829.76

3829.77

3812.27

3811.32

3810.03

$\begin{array}{ll}38 & 9.25\end{array}$

$\begin{array}{ll}38 & 8.79\end{array}$

$\begin{array}{ll}38 & 0.21\end{array}$

38 8.35

$\begin{array}{ll}38 & 8.07 \\ 38 & 6.78\end{array}$

$\begin{array}{ll}38 & 6.78\end{array}$

$\begin{array}{ll}38 & 5.87 \\ 38 & 5.53\end{array}$

$38 \quad 5.75$

$38 \quad 9.79$

$\begin{array}{ll}38 & 9.35 \\ 38 & 8.85\end{array}$

$\begin{array}{ll}38 & 8.85 \\ 38 & 8.25\end{array}$

387.66

$\begin{array}{ll}38 & 7.42\end{array}$

$38 \quad 7.62$

$\begin{array}{ll}38 & 7.72 \\ 38 & 7.72\end{array}$

$\begin{array}{ll}38 & 7.72 \\ 38 & 8.43\end{array}$

80.9 ?

811.15

$\begin{array}{lll}38 & 11.62\end{array}$

$\begin{array}{lll}38 & 12.02\end{array}$

$\begin{array}{lll}38 & 12.23 \\ 38 & 12.09\end{array}$

\section{EAST +}

$-8111.56$

$-8114.16$

$\begin{array}{ll}-81 & 14.19 \\ -81 & 13.93\end{array}$

-81 14.21

-81 14.12

8113.00

-81 13.55

$\begin{array}{ll}-81 & 13.14 \\ -81 & 12.50\end{array}$

$-8111.48$

-81 10.61

8111.52

817.82

$-81.10 .30$

$81 \quad 8.85$

8.

8.71

810.22

$-81 \quad 8.61$

$-8111.99$

-81 12.85

-8) 12.96

$-8133.23$

$-8114.02$

-81 14.00

-81 15.29

-81 15.29

$\begin{array}{ll}-81 & 17.25\end{array}$

-81 28.10

$\begin{array}{lll}-81 & 17.82\end{array}$

$\begin{array}{lll}-81 & 16.61\end{array}$

$-8115.95$

-81
-85.18

\section{EVATIO
FEET}

710.000 1014.000 1015.000 934.000 917.000 11.000 1103.000 652.000 650.000 640.000 670.000 715.000 693.000 00.000 680.000 702.000 902.000 1125.000 1140.000 1175.000 801.000 1175.000

1370.000

1400.000

620.000

625.000

668.000

698.000

680.000

665.000

670.000

655.000

655.000

676.000
642.000

650.000

686.000

768.000

850.000

908.000 MGN

\subsection{0}

.310

MGAL

PREE AIR
MGAL

BOUGU

THEOR G

C BOUGuer MGNL

.320

979915.860 979919.792

-29.535
-11.537

$-11.537$

980012.178 979926.341

$.470 \quad 979928.344$

- 10 979

$.380 \quad 979919.699$

$.560 \quad 979947.942$

$.650 \quad 979947.137$

$.780 \quad 979947.624$

$.250 \quad 979947.623$

.290979913 .521

$.350 \quad 979911.989$

$.280 \quad 979910.922$

$.470 \quad 979910.137$

$.550 \quad 979905.656$

$.370 \quad 979895.608$

$.450 \quad 979881.482$

$.410 \quad 979881.389$

$.470 \quad 979876.531$

$.560 \quad 979895.528$

$.350 \quad 979876.523$

.250 979865

$.320 \quad 979862.923$

$.470 \quad 979910.276$

.21097990 .605

$.190 \quad 979907.788$

.220979906 .212

. 150 979 97.894

$.270 \quad 979905.571$

$.190 \quad 979905.313$

$0.000 \quad 979905.990$

$.220 \quad 979907.234$

$.310 \quad 979910.610$

$.190 \quad 979914.545$

$.120 \quad 979913.692$

$.210 \quad 979909.355$

.310
$.210 \quad 979905.632$
$-14.685$

(5.031

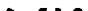

26.082

$-26.987$

(27.001

$-30.598$

$-32.881$

-31.898

4.675

$-24.507$

$-16.808$

$-15.695$

$-16.851$

-31.145

$-5.463$

$-5.899$

$-37.028$

$-38.385$

$-34.425$

$-34.440$

$-34.831$

$-34.911$

35.792

$-33.612$

$-34.224$

$-32.729$

$-30.884$

$-28.094$

-24.412
-23.673
-53.751
-46.122 980026.707

(1)

4.54900079

4.907900031 .755

$-47.38190031 .755$

$-46.13290031 .961$

$-48.320 \quad 980035.352$

49.157900035

49.709980035 .704

$-47.927980035 .729$

$-54.985980011 .373$

.56.517 980010.054

$-55.773 \quad 980008.663$

$-55.06898006 .774$

-57.080 980005.632

$-55.2729$

$-55.179 \quad 980004.109$

$-51.57798004 .314$

$-56.927980003 .904$

$-58.46598002 .016$

$-53.71598000 .684$

$-53.650 \quad 980000.509$

$-58.97598006 .422$

$-59.702980005 .778$

56.2089005 .046

56.10898004 .168

57.512980002 .353

-57.763 900003.946

57.76390003 .246

$-58.13290003 .392$

56.66920000 .392

56.1219000 .222

$-54.2819000 .414$

$-54.281980009 .102$

$-53.403 \quad 960009.996$

-53.4043
-54.64380009 .791

$-53.751$

45.714

46.051

$-46.437$

6.87

$-45.752$

$-48.507$

48.929

$-47.677$

$-54.695$

$-56.167$

(1)

$-57.338$

54.302

$-54.729$

$-54.167$

5.905

$-53.365$

$-51.939$

$-58.505$

$-59.492$

55.888

$-57.491$

$-57.573$

57.573

$-58.132$

$-55.811$

$-54.709$

$-54.161$

$-54.079$

$-54.433$
56.121900005 .222 
PRINCIPAL PACTEATERAVITY STATION

STA NAME AND NUM

\begin{tabular}{|c|c|c|c|}
\hline $\begin{array}{l}1.202 \\
1.216 \\
1.222 \\
1.201 \\
1.217 \\
1.218 \\
1.219 \\
1.220 \\
1.221 \\
1.203 \\
1.204 \\
1.205 \\
1.206 \\
1.409 \\
1.410 \\
1.411 \\
1.412 \\
1.413 \\
1.414 \\
1.415 \\
1.416 \\
1.115 \\
1.114 \\
1.113 \\
1.116 \\
1.117 \\
1.118 \\
1.112 \\
1.111 \\
1.110 \\
1.109 \\
12.203 \\
12.202 \\
12.201 \\
1.209 \\
1.201 \\
1.108 \\
1.107 \\
1.106 \\
1.105\end{array}$ & $\begin{array}{l}536 \\
537 \\
538 \\
539 \\
540 \\
541 \\
542 \\
543 \\
544 \\
545 \\
546 \\
547 \\
548 \\
549 \\
550 \\
551 \\
552 \\
553 \\
554 \\
555 \\
556 \\
557 \\
558 \\
559 \\
560 \\
561 \\
562 \\
563 \\
561 \\
565 \\
566 \\
567 \\
568 \\
569 \\
570 \\
2075 \\
571 \\
572 \\
573 \\
574\end{array}$ & $\begin{array}{l}38 \\
38 \\
38 \\
38 \\
38 \\
38 \\
38 \\
38 \\
38 \\
38 \\
38 \\
38 \\
38 \\
38 \\
38 \\
38 \\
38 \\
38 \\
38 \\
38 \\
38 \\
38 \\
38 \\
38 \\
38 \\
38 \\
38 \\
38 \\
38 \\
38 \\
38 \\
38 \\
38 \\
38 \\
38 \\
38 \\
38 \\
38 \\
38 \\
38\end{array}$ & $\begin{array}{l}11.00 \\
11.05 \\
11.32 \\
11.61 \\
12.52 \\
12.89 \\
12.68 \\
13.04 \\
13.41 \\
10.47 \\
9.87 \\
8.68 \\
8.12 \\
7.43 \\
6.36 \\
5.95 \\
5.55 \\
5.56 \\
4.37 \\
3.86 \\
3.52 \\
12.85 \\
12.49 \\
13.26 \\
13.78 \\
14.31 \\
14.75 \\
13.23 \\
12.68 \\
12.25 \\
11.00 \\
12.25 \\
12.90 \\
14.56 \\
8.19 \\
11.61 \\
11.59 \\
10.92 \\
10.65 \\
10.36\end{array}$ \\
\hline
\end{tabular}

\section{EAST +}

$-81 \quad 19.02$

$-8120.56$

$-8121.36$

$\begin{array}{lll}-81 & 22.14 \\ -81 & 22.36\end{array}$

$-8121.30$

$\begin{array}{ll}-81 & 21.30 \\ -81 & 20.63\end{array}$

- 8119.93

$\begin{array}{ll}-81 & 19.81\end{array}$

$\begin{array}{lll}-81 & 18.30\end{array}$

$\begin{array}{ll}-81 & 18.53 \\ -81 & 17.61\end{array}$

$\begin{array}{ll}-81 & 17.61 \\ -81 & 18.21\end{array}$

-81 18.21

-81 18.62

$-8119.40$

-81 19.50

$\begin{array}{ll}-81 & 19.95 \\ -81 & 20.00\end{array}$

-81 20.00

-81 20.35

-81 20.35

-81 20.25

-81 25.97

-8) 25.78

-81 25.75

- 24.25

-81 24.40

- 8123.35

$-8112.89$

$-8113.61$

$-8114.75$

$\begin{array}{ll}-81 & .16\end{array}$

$\begin{array}{ll}-81 & 1.01\end{array}$

-81 16.3

$\begin{array}{ll}-81 & 16.3 \\ -81 & 22.1\end{array}$

$\begin{array}{ll}-81 & 22.14\end{array}$

$\begin{array}{ll}-81 & 13.73 \\ -81 & 13.11\end{array}$

-81
-812.89

$\begin{array}{ll}-81 & 12.89 \\ -81 & 22.07\end{array}$
ELEVATION

FEET

640.000

612.000

640.000

610.000

672.000

740.000

800.000

804.000

680.000

620.000

642.000

655.000

825.000

805.000

834.000

880.000
930.000

1036.000

$\$ 126.000$

1173.000

642.000

652.000

620.000

628.000

667.000

721.000

634.000
632.000

632.000

620.000

620.000

621.000

643.000

611.000

670.000

640.000

607.000

633.000
640.000

640.000
640.000
RR

$$
\text { MGAL }
$$$$
\text { SPRV }
$$

$$
\text { MGNL }
$$

PREE

MGNL

$-33.328$

$-31.024$

$-28.95$

$-30.477$

$-27.732$

$-24.113$

$-21.848$

$-23.810$

$-32.413$

$-36.998$

$-34.901$

$-35.698$

$-33.621$

$-29.978$

$410 \quad 97989$

$360 \quad 979892$

$470 \quad 979887.539$

10979884.488

$490 \quad 979877.849$

$380 \quad 979872.679$

$.650 \quad 979868.763$

.620979922 .231

.710

.390 979

$410 \quad 979923.844$

$270 \quad 979921.049$

97921.049

$510 \quad 979922.180$

$.270 \quad 979922.279$

$.280 \quad 979921.665$

.310979222 .859

.360979924 .838

0.000979907 .406

$0.000 \quad 979920.590$

$460 \quad 979923.277$

$390 \quad 979920.803$

$.510 \quad 979920.680$

$.480 \quad 979918.367$
OUGUER

THEOR 6

MGAL

C Bouguer

MGNL

$-55.157 \quad 980008.194 \quad-54.797$ $-52.921000000 .260-52.551$ $-52.57200000 .6630-52.202$

$-50.544900009 .080 \quad-50.154$

$-51.282090010 .421 \quad-50.932$

$-50.652980010 .963 \quad-50.262$

$-49.353 \quad 980010.655 \quad-48.903$

$-49.134 \quad 980011.182 \quad-48.714$

$-51.232 \quad 980011.724 \quad-50.612$
-55.606

$\begin{array}{lll}-55.606 & 980007.418 & -54.896\end{array}$

$-58.145 \quad 980006.540 \quad-57.395$

$\begin{array}{llll}-56.798 & 980004.797 \quad-56.108\end{array}$

$\begin{array}{lll}-58.038 & 980003.977 \quad-57.528\end{array}$

$\begin{array}{lll}-58.349 & 980002.967 \quad-57.859\end{array}$

$-57.434 \quad 980001.401 \quad-57.024$

$-58.370 \quad 980000.801 \quad-58.010$

$-59.917 \quad 980000.216 \quad-59.447$

$\begin{array}{lll}-59.986 & 980000.231 & -59.476\end{array}$

$-58.528-979998.489-58.038$

$-57.555 \quad 979997.743 \quad-57.175$

$-58.157-979997.246 \quad-57.707$

$-50.183090010 .904-49.563$

$-48.363980010 .377-47.653$

$-48.656 \quad 980011.505-40.266$

$-50.77200012 .266-50.362$

$-48.477900013 .043 \quad-47.987$

$-49.412090013 .680 \quad-49.142$

-49.412
-49.605

$-50.584 \quad 980010.655 \quad-50.074$

$-50.575 \quad 980010.025 \quad-50.305$

$-49.357980008 .294 \quad-49.077$

$-50.252 \quad 980010.025 \quad-50.012$

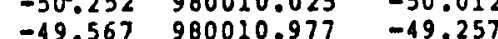

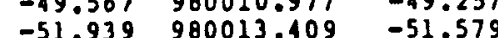

$-56.505980004 .080 \quad-56.505$

$-50.527 \quad 980009.088 \quad-50.127$

$-49.389 \quad 980009.058 \quad-48.929$

$\begin{array}{lll}-49.389 & 980009.058 & -48.929 \\ -49.323 & 980008.077 & -48.933\end{array}$

$\begin{array}{lll}-48.631 & 980007.682 & -48.931\end{array}$

$\begin{array}{lll}-48.631 & 980007.682 & -48.121 \\ -50.519 & 980007.257 & -50.039\end{array}$ 
PRINCIPAL PACTEATTGRAVITY STATION

STA NAME AND NUM

\begin{tabular}{ll}
1.104 & 575 \\
1.103 & 576 \\
1.101 & 578 \\
1.389 & 579 \\
1.317 & 580 \\
1.301 & 581 \\
1.316 & 582 \\
1.309 & 583 \\
1.314 & 584 \\
1.308 & 585 \\
1.307 & 586 \\
1.306 & 587 \\
1.315 & 588 \\
1.305 & 589 \\
1.304 & 590 \\
1.303 & 591 \\
1.302 & 592 \\
1.301 & 593 \\
1.331 & 594 \\
1.312 & 595 \\
1.318 & 596 \\
1.401 & 597 \\
1.402 & 598 \\
1.313 & 599 \\
1.402 & 600 \\
1.403 & 601 \\
1.404 & 602 \\
1.405 & 603 \\
1.406 & 604 \\
1.407 & 605 \\
1.119 & 606 \\
1.120 & 607 \\
1.121 & 608 \\
1.222 & 609 \\
3.304 & 610 \\
3.305 & 611 \\
3.306 & 612 \\
3.307 & 613 \\
3.308 & 614 \\
3.309 & 615 \\
1309 & \\
\hline
\end{tabular}

LATITUDE

$38 \quad 10.04$

$38 \quad 9.48$

$\begin{array}{ll}38 & 7.69 \\ 38 & 7.42\end{array}$

38 3 7.42

$38 \quad 6.40$

$\begin{array}{ll}38 & 6.37 \\ 38 & 5.42\end{array}$

38 5. 382

$38 \cdot 4.07$

83.42

3.84

$\begin{array}{ll}38 & 2.30\end{array}$

$38 \quad 1.92$

$38 \quad 1.56$

38 1.09

$38 \quad .34$

$38 \quad 7.10$

3806.05

$38 \quad 3.45$

$38 \quad 2.82$

38 4.85

$\begin{array}{ll}38 & 2.82 \\ 38 & 2.35\end{array}$

$\begin{array}{ll}38 & 1.18\end{array}$

$\begin{array}{ll}-81 & 21.07 \\ -81 & 20.83\end{array}$

$8.47-8124.34$

$9.43=81$

$\begin{array}{llll}38 & 8.48 & -81 & 24.35\end{array}$

$\begin{array}{llll}38 & 10.81 & -81 & 23.80\end{array}$

$\begin{array}{llll}38 & 19.97 & -81 & 29.5\end{array}$

$\begin{array}{llll}38 & 20.52 & -81 & 29.13\end{array}$

$\begin{array}{llll}38 & 20.37 & -81 & 28.0\end{array}$

$\begin{array}{llll}38 & 19.82 & -81 & 27.13\end{array}$

$\begin{array}{llll}38 & 19.65 & -81 & 26.21 \\ 38 & 19.26 & -81 & 25.57\end{array}$

\section{FEET}

656.000
670.000

670.000
720.000

720.000
731.000

731.000
790.000

790.000

777.000

B12.000

846.000

885.000

930.000

988.000

1070.000

1080.000

1080.000

1124.000

1300.000

1385.000

733.000

762.000
800.000
880.000

880.000

880.000

800.000

880.000

888.000

930.000

950.000

980.000
1030.000

636.000

666.000

636.000

625.000

659.000

660.000

690.000

734.000

760.000 CORA MCAL

MGAL

BOUGUER

THEOR 6
MGAL

$-50.102 \quad 980006.788$

$.450 \quad 979917.356$

$-051-27.728$
-25.896

$.430 \quad 979910.485 \quad-25.139$

$.710 \quad 979909.216 \quad-24.978$

$.690 \quad 97905.348 \quad-22.608$

.710 979904.437 -22.715

$.650 \quad 979903.944-24.386$

$.720 \quad 979900.552 \quad-23.096$

$.700 \quad 979896.425 \quad-23.074$

$.730 \quad 979894.292 \quad-20.514$

$.620 \quad 979888.989$

.170979884 .301

$.390 \quad 979880.337$

$.420 \quad 979877.138$

$.440 \quad 979874.268$

$.370 \quad 979872.543$

$.590 \quad 979867.156$

$.490 \quad 979861.114$

$.470 \quad 979903.327$

$.480 \quad 979900.822$

$.510 \quad 979898.610$

$.570 \quad 979888.679$

$.480 \quad 979887.327$

$0.000 \quad 979895.158$

$.570 \quad 979887.327$

$.620 \quad 979884.677$

.710979880 .411

$.850 \quad 979879.062$

.79097987 .654

$.690 \quad 979872.238$

$.490 \quad 979915.392$

$.410 \quad 979913.573$

$.420 \quad 979915.396$

.510979918 .799

$.390 \quad 979927.868$

$.270 \quad 979927.409$

$.250 \quad 979926.561$

$.350 \quad 979924.212$

.390
$.290 \quad 979920.532$
$-49.696 \quad 980003.348$

$49.910 \quad 980002.953$

$49.553 \quad 980002.265$

659980001.460

$.887 \quad 980001.416$

$-50.791 \quad 980000.026$

.51.929 979999.075

.50.69 9

$-52.353 \quad 979997.100$

-54.178

$-50.974 \quad 979995.461$

$-53.017979994 .906$

$-55.36197994 .379$

$-53.761 \quad 979993.692$

$-48.390 \quad 979993.487$

$-48.444979992 .595$

$-55.211980002 .484$

$-54.440 \quad 980000.948$

$-53.936980000 .509$

-55.705 97999.144

-56.135 97996.222

-56.070 97999.192

-56.135 979 96.222

$-57.61897959 .534$

-57.108

57.005 979

-57.814 979

58.326 979 922.317

$-50.96798004 .490$

$-52.393 \quad 98005.895$

$-51.646 \quad 980007.916$

$-53.961980021 .339$

$-55.167980022 .146$

$-53.996 \quad 980021.926$

$-52.901980021 .119$

$-52.248 \quad 980020.870$

$-52.222980020 .298$
$-50.977980004 .504$
T C Bouguer

$-49.652$

$-48.418$

$-49.200$

4

50.07

$-51.229$

$-19.969$

$-51.733$

$-50.584$

$-52.597$

-

$-47.954$

$-54.741$

$-55.135$

55.655

$-56.070$

$-56.098$

57.398

$-57.024$

$-57.636$

51.47

$-50.557$

$-51.136$

$-54.897$

$-53.746$

$-52.551$

$-51.932$ 
PRINCIPAL PACTSAT GRAVITY BTATIONS

STA NAME AND NUM

$\begin{array}{ll}3.310 & \\ 3.311 & 616 \\ 3.303 & 617 \\ 3.301 & 618 \\ 3.401 & 619 \\ 3.402 & 620 \\ 3.403 & 621 \\ 3.404 & 622 \\ 3.405 & 623 \\ 3.406 & 624 \\ 3.407 & 625 \\ 3.416 & 626 \\ 3.415 & 627 \\ 3.421 & 628 \\ 3.422 & 629 \\ 3.423 & 630 \\ 3.414 & 631 \\ 3.408 & 632 \\ 3.413 & 633 \\ 3.408 & 634 \\ 3.424 & 1074 \\ 3.312 & 635 \\ 3.103 & 636 \\ 3.199 & 637 \\ 3.104 & 638 \\ 3.1105 & 640 \\ 3.313 & 641 \\ 3.314 & 642 \\ 3.102 & 643 \\ 3.101 & 644 \\ 3.411 & 645 \\ 3.410 & 646 \\ 3.238 & 647 \\ 3.239 & 648 \\ 2.237 & 649 \\ 3.240 & 650 \\ 3.419 & 651 \\ 3.418 & 652 \\ 3.417 & 653 \\ 3.424 & 654 \\ & \end{array}$

LATITUDE
NORTH +

$\begin{array}{ll}38 & 18.83 \\ 38 & 18.39\end{array}$

$38 \quad 15.17$

38 3 15.62

3815.84

$\begin{array}{lll}38 & 16.06\end{array}$

$38 \begin{array}{lll}16.32 \\ 38 & 16.35\end{array}$

38 16.35

$38 \quad 17.46$

3818.02

3818.65

38 3 38.98

$38 \quad 18.99$

3819.50

$38 \quad 20.15$

$\begin{array}{ll}38 & 20.62 \\ 38 & 20.15\end{array}$

3821.48

$38 \quad 22.30$

$\begin{array}{ll}38 & 23.04 \\ 38 & 23.05\end{array}$

3823.48

3823.48

$\begin{array}{ll}38 & 22.37 \\ 38 & 21.87\end{array}$

3021.87

3822.74

3821.87

$\begin{array}{lll}38 & 21.48\end{array}$

$\begin{array}{lll}38 & 22.35\end{array}$

3823.00

3822.64

3821.19

3821.92

$\begin{array}{ll}38 & 21.78 \\ 38 & 21.67\end{array}$
LONG ITUDE
EAST +

-81 25.63

$\begin{array}{ll}-81 & 25.29\end{array}$

$-8115.20$

$\begin{array}{ll}-81 & 15.19\end{array}$

0122.43

-81 21.39

$-8119.02$

$\begin{array}{lll}-81 & 18.13\end{array}$

$\begin{array}{ll}-81 & 17.52 \\ -81 & 17.81\end{array}$

-81 17.31

$\begin{array}{ll}-81 & 17.51\end{array}$

$-81 \quad 17.62$

$\begin{array}{ll}-81 & 16.93 \\ -81 & 16.19\end{array}$

$\begin{array}{ll}-81 & 16.19 \\ -81 & 18.56\end{array}$

$-8118.56$

-81 29.12

$-8118.82$

$\begin{array}{ll}-81 & 21.11\end{array}$

-81 25.19

-81 25.83

$\begin{array}{lll}-81 & 27.10\end{array}$

$\begin{array}{lll}-81 & 27.87\end{array}$

$\begin{array}{ll}-81 & 24.20\end{array}$

-81 24.27

-81 24.61

$\begin{array}{ll}-81 & 22.74\end{array}$

$\begin{array}{ll}-81 & 22.02\end{array}$

B) 21.60

$-8119.98$

-81 17.8

-81 15.30

- 16.54

-81 16.54

$-8117.80$

$-8118.55$ bre?

.850 .000

.820 .000

780.000

880.000

880.000

1005.000

2160.000

1345.000

1320.000

1180.000

1076.000

1000.000

946.000

1060.000

900.000

859.000

840.000

859.000

760.000

1052.000

1052.000

784.000

729.000

687.000

720.000

690.000

698.000

720.000

080.000

1080.000

1386.000

1396.000

850.000

860.000

820.000
GRP$$
\text { MGAL }
$$

OBSFRVED
MCAL

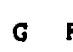

$G$ PREF AIR

MGNL

BOUGUER
MGAL

THEOR

MGAL

T C BOUGUER
MGAL

$.310 \quad 979918.742$

$-23.795$

$-23.331$

$-17.296$

$-23.705$

$-18.359$

$-18.490$

$-15.032$

$-2.106$

$-2.181$

$-7.371$

$-13.367$

$-14.649$

-16.108
-8.671

$-10.190$

$-18.560$

$-19.121$

$-20.357$

$-18.894$

$-24.410$

$-25.684$

$-16.198$

$-16.213$

$-26.859$

$-29.437$

$-28.06$

$-24.955$

$-28.482$

$-28.128$

$-26.284$

$-26.959$

$-14.952$

.441

$-51.763 \quad 980019.668$

$-52.323 \quad 980019.022$

$-45.264980014 .303$

$-50.309980014 .962$

$-48.373980015 .153$

40.504980015 .285

980015.607

980015.98

$-47.202 \quad 980026.941$

$-47.617 \quad 980017.65$

$-50.066980018 .480$

$-48.756 \quad 980019.404$

$-48.373980019 .887$

$-45.507980020 .181$

$-46.344 \quad 980019.902$

$-49.256 \quad 980020.650$

980021.603

$49.007 \quad 980022.292$

48.192980021 .603

$-50.332980023 .553$

$-52.560 \quad 980024.756$
-52.079

$-52.079 \quad 980025.842$

$-52.093 \quad 980025.856$

$-53.600 \quad 980026.487$

$-54.301980026 .487$

$-51.492 \quad 980024.859$

$-49.512 \quad 980024.125$

$-52.016 \quad 980024.830$

$-51.934980025 .402$

$-50.842 \quad 980024.125$

$-51.994980023 .553$

$-51.788 \quad 980024.830$

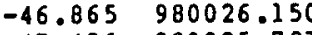

$-47.456 \quad 980025.783$

$-48.817980025 .255$

$-46.699 \quad 980023.128$
-47.117

$-47.117 \quad 980024.199$

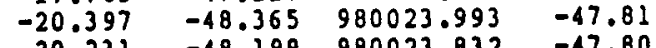

$\begin{array}{llllll}.550 & 979926.466 & -20.397 & -48.365 & 980023.993 & -47.815 \\ .390 & 979926.471 & -20.231 & -48.199 & 980023.832 & -47.809\end{array}$

$-51.453$

$-51.903$

$-14.884$

$-49.949$

$-48.043$

$-48.034$

$-48.689$

$-47.570$

$-46.462$

$-47.167$

$-49.516$

$-47.55$

$-44.817$

$-45.604$

$-48.456$

$-47.529$

$-51.810$

$-51.363$

$-52.760$

$-53.411$

$-48.992$

$-51.416$

$-51.424$

$-50.362$

$-51.368$

$-46.375$

$-46.896$

$-48.347$

$-46.179$

$-46.627$ 
PRINCIPAL PACTSAT GRAVITY STATIONB

STA NAME AND NUM

1.142
3.228
3.236
3.227
3.229
3.230
3.231
3.232
3.233
3.234
3.235
3.208
3.209
3.207
3.206
3.205
3.204
3.203
3.202
3.201
3.223
3.224
3.225
3.210
3.211
3.212
3.213
3.214
3.215
3.216
3.217
3.218
3.219
3.220
3.221
3.222
3.223
3.1105
3.106
3.199
LATITUDE

$38 \quad 22.89$

$\begin{array}{ll}38 & 24.30\end{array}$

38 3 34.92

$38 \quad 25.10$

$\begin{array}{ll}38 & 25.93\end{array}$

$38 \quad 26.10$

$\begin{array}{ll}38 & 26.97 \\ 38 & 27.62\end{array}$

3828.48

$\begin{array}{ll}38 & 28.89 \\ 38 & 29.38\end{array}$

$\begin{array}{ll}38 & 29.38 \\ 38 & 29.44\end{array}$

$38 \quad 29.55$

3828.54

$\begin{array}{ll}38 & 28.32 \\ 38 & 29.05\end{array}$

3829.31

$\begin{array}{ll}38 & 28.93\end{array}$

$\begin{array}{ll}38 & 29.55 \\ 38 & 27.56\end{array}$

$\begin{array}{lll}38 & 27.66\end{array}$

3827.39

3828.98

3828.27

$38 \quad 27.98$

3827.73

3826.88

3826.46

3825.80

3825.47

3824.66

3824.12

$38 \quad 23.58$

$\begin{array}{ll}38 & 23.20\end{array}$

3823.48

$\begin{array}{ll}38 & 24.03 \\ 38 & 24.92\end{array}$
LONG I TUDE
EAST +

-81 14.48

-81 15.85

-81 15.36

8116.69

$-8116.99$

$-8117.82$

-81 18.5

0218.79

$-8118.78$

- 21.39

$\begin{array}{ll}-81 & 20.39\end{array}$

$-8118.90$

8117.80

-81 16.29

8115.72

$\begin{array}{ll}-81 & 15.03\end{array}$

8116.35

$-8116.38$

810.38

21.79

$-8121.37$

-81 20.08

$-8120.87$

$\begin{array}{ll}-81 & 21.28 \\ -81 & 21.73\end{array}$

-81 22.02

$\begin{array}{ll}-81 & 22.32\end{array}$

$\begin{array}{ll}-81 & 22.34\end{array}$

$\begin{array}{ll}-81 & 27.79\end{array}$

$\begin{array}{ll}-81 & 28.97\end{array}$

\section{FEET
FET}

1214.000

1000.000

1080.000

1080.000
980.000

980.000
1209.000

915.000

820.000

780.000

664.000

640.000

635.000

629.000

625.000

650.000

640.000

650.000

640.000

667.000

638.000

880.000

954.000

880.000

745.000

745.000

661.000

797.000

714.000

757.000

800.000

1001.000

748.000

1207.000

1100.000

2175.000

1171.000

729.000

694.000
ERA CORA MGAL
MGNL

$-4.684$

$.470 \quad 979906.747$

$.710 \quad 979919.986$

$.510 \quad 97908.411$

$.670 \quad 979909.693$

$.820 \quad 979923.104$

$.490 \quad 979909.434$

$.920 \quad 979929.380$

$.960 \quad 979933.245$

$1.200 \quad 979943.126$

1.020979943 .811

$.420 \quad 979945.860$

.350 979945.22

.

$.270 \quad 979944.730$

$.350 \quad 979945.553$

0

$.410 \quad 979945.563$

$.380 \quad 979931.702$

$.350 \quad 979926.695$

$.280 \quad 979924.993$

.420979936 .999

$.380 \quad 979940.340$

$.210 \quad 979932.367$

$.190 \quad 979937.128$

.330979934 .496

$.170 \quad 979932.782$

979919.084

979933.557

$.150 \quad 979907.416$

$.340 \quad 979904.888$

$.380 \quad 979912.482$

$.420 \quad 979905.417$

$.440 \quad 979905.054$

$.150 \quad 979930.847$

$.160 \quad 979937.365$
$-13.643$

$-17.136$

$-13.579$

$-6.927$

$-18.414$

$-25.098$

$-25.950$

$-29.558$

$-29.558$

$-31.386$

$-28.847$

$-28.660$

$-27.969$

$-27.35$

$-18.145$

$-15.796$

$-23.827$

-27.485

$-26.443$

$-24.980$

$-28.436$

$-25.776$

$-22.829$

$-16.652$

$-25.492$

$-9.549$

$-11.476$

$-10.695$

-10.876
-29.436

$-31.168$

$-34.798$
BOUGUER

HEOR $O$

MGAL

C C BOUGUER

MGAL

$-46.090 \quad 980025.622$
$-47.0750 \quad 980027.690$

$\begin{array}{lll}-53.971 & 980027.133 & -53.461\end{array}$

$\begin{array}{lll}-53.848 & 980028.292 \quad-53.178\end{array}$

$-47.004 \quad 980028.864 \quad-46.184$

$-48.163 \quad 980030.082-47.673$

$-49.622980030 .332-48.952$

$-53.066 \quad 980031.608-52.146$

$-52.554 \quad 980032.563-51.594$
-50.060

$-50.850 \quad 980033.825-$
-49.690

$-52.245 \quad 980034.427-51.225$

$-51.216 \quad 980035.146-50.796$

$-52.820-980035.234-52.450$

$\begin{array}{lll}-52.703 \quad 980035.396 & -52.453\end{array}$

\begin{tabular}{l}
$-51.017 \quad 980033.913-50.637$ \\
\hline
\end{tabular}

$\begin{array}{lll}-50.489 & 980033.590 \quad-50.219\end{array}$

$\begin{array}{lll}-50.139 & 980034.662 & -49.789\end{array}$

$-49.745 \quad 980035.044 \quad-49.415$

$\begin{array}{lll}-48.933 & 980034.486 & -48.523\end{array}$

$-49.117 \quad 980035.396 \quad-48.947$

$\begin{array}{lll}-48.159 & 980032.621 & -47.779\end{array}$

$\begin{array}{lll}-48.334 & 980032.225 & -47.984\end{array}$

$\begin{array}{lll}-53.841 & 980031.594 & -53.561\end{array}$

$-51.053 \quad 900033.517-51.583$

$-53.122 \quad 980033.010-51.583$

$-52.163 \quad 980032.313-51.953$

$-52: 708$ 900032.724 -51.953

$-52.780-980032.724-52.598$
-50.595

$-51.595 \quad 980031.476-51.265$

$-50.115 \quad 980030.860-49.945$

$\begin{array}{lll}-50.793 & 980029.891 & -50.543\end{array}$

$\begin{array}{lll}-51.004 & 980029.407 & -50.724\end{array}$

$\begin{array}{lll}-50.716 & 980028.218 & -50.566\end{array}$

$\begin{array}{lll}-50.716 & 980027.969 & -50.376\end{array}$

$\begin{array}{lll}-48.994 & 980027.426 & -48.614\end{array}$

$\begin{array}{lll}-50.771 & 980026.634 & -50.351\end{array}$

$\begin{array}{lll}-50.816 & 980026.076 & -50.376\end{array}$

$\begin{array}{lll}-54.301 & 980026.487 & -53.911\end{array}$

$\begin{array}{lll}-54.839 & 980027.294 & -54.689\end{array}$ 
PRINCI PAL PACTBATTGRAVITY ETATION B

STA NAME AND NUM

\begin{tabular}{lr}
3.107 & \\
3.108 & 694 \\
3.109 & 695 \\
3.110 & 696 \\
3.111 & 697 \\
3.112 & 698 \\
3.127 & 699 \\
3.125 & 700 \\
3.124 & 701 \\
3.123 & 702 \\
3.122 & 703 \\
3.121 & 704 \\
3.120 & 705 \\
3.118 & 706 \\
3.119 & 707 \\
3.105 & 708 \\
3.112 & 1079 \\
3.130 & 709 \\
3.113 & 710 \\
3.114 & 711 \\
3.115 & 712 \\
3.116 & 713 \\
3.117 & 714 \\
13.118 & 715 \\
13.416 & 716 \\
13.415 & 718 \\
13.411 & 719 \\
13.412 & 720 \\
13.413 & 721 \\
13.414 & 723 \\
13.318 & 724 \\
13.316 & 725 \\
13.317 & 726 \\
13.101 & 727 \\
13.114 & 728 \\
13.102 & 729 \\
13.113 & 730 \\
13.103 & 731 \\
13.104 & 732 \\
13.105 & 733 \\
& \\
\hline
\end{tabular}

LATITUDE NORTH +

$38 \quad 25.69$

$38 \quad 26.38$

$38 \quad 26.67$

38 26.70

3027.00

8 26.83

3826.09

$\begin{array}{ll}38 & 25.80\end{array}$

$38,25.26$

3824.52

$\begin{array}{lll}38 & 24.10\end{array}$

$\begin{array}{ll}38 & 23.42\end{array}$

$38 \quad 22.80$

$\begin{array}{ll}38 & 23.41\end{array}$

38 . 27.38

38 . 27.33

$\begin{array}{ll}38 & 28.02\end{array}$

$\begin{array}{lll}38 & 27.67\end{array}$

$\begin{array}{ll}38 & 27.73\end{array}$

$\begin{array}{lll}38 & 28.33\end{array}$

828.50

3831.85

3832.38

38 32.69

3833.61

3834.10

$\begin{array}{lll}38 & 35.59\end{array}$

3837.22

$\begin{array}{ll}38 & 37.98\end{array}$

$38 \quad 38.35$

$38 \quad 38.75$

3839.69

$38 \quad 40.26$

\section{EAST +}

$-8129.20$

$\begin{array}{lll}-81 & 29.11 \\ -81 & 28.29\end{array}$

8128.01

$\begin{array}{ll}-81 & 27.42\end{array}$

-81 27.45

$-8126.43$

$\begin{array}{ll}-81 & 25.72\end{array}$

$-8125.50$

-81 25.06

$\begin{array}{ll}-81 & 25.59\end{array}$

$\begin{array}{ll}-81 & 24.35\end{array}$

$\begin{array}{lll}-81 & 25.21\end{array}$

$\begin{array}{ll}-81 & 27.79\end{array}$

$\begin{array}{ll}-81 & 27.14\end{array}$

$\begin{array}{lll}-81 & 26.71 \\ -81 & 26.31\end{array}$

-81 25.40

$\begin{array}{ll}-81 & 25.59\end{array}$

$\begin{array}{ll}-81 & 24.00 \\ -81 & 23.29\end{array}$

3.29
21.55

-81 20.87

$\begin{array}{ll}-81 & 21.32 \\ -81 & 21.49\end{array}$

$-8121.55$

$\begin{array}{ll}-81 & 21.62\end{array}$

$\begin{array}{ll}-81 & 22.35 \\ -81 & 22.78\end{array}$

-81 22.58

$-8123.35$

$\begin{array}{lll}-81 & 23.62\end{array}$

$\begin{array}{ll}-81 & 24.15 \\ -81 & 24.66\end{array}$

-81 23.69

$\begin{array}{ll}-81 & 23.82\end{array}$

$\begin{array}{ll}-81 & 23.55 \\ -81 & 23.22\end{array}$
FEET
FETION

619.000

610.000

612.000

620.000

625.000

624.000

617.000

634.000

636.000

672.000

666.000

652.000

826.000

686.000

681.000

729.000

683.000

610.000

603.000

638.000

651.000

607.000

620.000

620.000

628.000

640.000

636.000

637.000

658.000

641.000

731.000

822.000

820.000

705.000

710.000

716.000

720.000

710.000

719.000

735.000
MGNL
ERR CORR MSPRVE
MGAL
PRFE AIR
MGNL

$.230 \quad 979937.362$

$.190 \quad 979938.545$

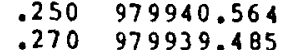

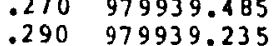

$.290 \quad 979939.235$

$\begin{array}{rr}.320 & 979939.233 \\ .250 & 979939.333\end{array}$

$.250 \quad 979939.333$

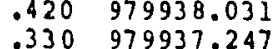

$.330 \quad 979937.247$

$.310 \quad 979935.128$

$.510 \quad 979935.014$

$.420 \quad 979935.813$

.59079923 .694

$.580 \quad 979933.454$

$0.000 \quad 979940.700$

$.590 \quad 979937.358$

$.620 \quad 979938.553$

$.670 \quad 979938.925$

$.270 \quad 979937.257$

$.390 \quad 979940.700$

$.280 \quad 979941.377$

$.460 \quad 979940.842$

$.400 \quad 979945.560$

$.480 \quad 979945.504$

$.510 \quad 979946.336$

$.590 \quad 979946.474$

$.490 \quad 979945.760$

$.460 \quad 979945.799$

$.380 \quad 979944.183$

$.510 \quad 979939.941$

$.290 \quad 979941.140$

$.630 \quad 979949.062$

$.590 \quad 979949.602$

$.580 \quad 979949.477$

$.270 \quad 979950.216$

$.250 \quad 979950.831$

$.190 \quad 979951.356$

$.280 \quad 979951.613$
$-34.144$

$-34.820$

$-33.038$

$-33.629$

$-33.725$

$-34.034$

0.651

$-30.762$

$-30.354$

$-30.256$

$-25.010$

$-29.405$

$-28.875$

30.535

$-37.014$

$-37.506$

$-35.368$

4.080

$-34.159$

$-37.513$

$-34.143$

$-33.848$

$-34.263$

$-33.707$

$-35.987$

-31.326
-28.449

$-28.394$

$-31.939$

$-31.619$

$-32.681$

$-32.146$

$-31.722$
OUGUER
MGAS

EOR G

$-55.256 \quad 980029.730$
-555.625

$-55.625980030 .743$

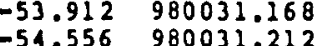

$-54.556 \quad 980031.212$

$-55.008 \quad 980031.652$

55.079900031 .403

-54.275980030 .317
-54.514

$-54.514980029 .891$

$-53.682980029 .099$

$-53.069980028 .013$

$-52.494 \quad 980027.397$
-53.183

$-53.183 \quad 980026.399$

$-52.802 \quad 980025.490$

$-52.102980026 .384$

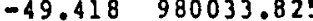

$-53.830 \quad 980032.137$
-57.819

$-57.819 \quad 980032.944$

$-58.073 \quad 980033.150$

$-57.128 \quad 980032.636$

$-56: 287 \quad 980032.724$

$-56.513980033 .605$

$-55.306 \quad 980033.854$

$-58.660 \quad 980036.673$
-55.562

$-55.562980038 .77$

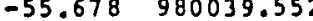

$-55.540 \quad 980040.007$
-55.989

$-55.989 \quad 980040.654$

$-56.149980041 .359$

$-57.849 \quad 980042.079$

$-56.258 \quad 980044.268$

$-56: 485 \quad 980045.708$

$-56.362980046 .664$

$-56.451980047 .781$

$-56.155980048 .325$

$-56.508980048 .913$

$-56.176 \quad 980049.560$

$-56.897980050 .295$

$\begin{array}{ll}-56.670 & 980051.133 \\ -56.791 & 980052.471\end{array}$
T C BOUCUER

$M G \wedge L$

$-55.026$

$-55.435$

$-53.662$

$-54.286$

$-54.656$

$-54.688$

$-54.829$

$-53.855$

$-54.184$

$-53.372$

-52.559
-52.074

$-52.793$

$-52.212$

$-51.522$

$-49.418$

$-53.240$

$-57.199$

$-57.403$

-56.858
-55.977

$-56.123$

$-55.026$

$-58.200$

$-55.162$

$-55.198$

55.030

$-55.399$

$-55.659$

$-57.389$

$-55.878$

$-55.975$

$-56.072$

$-55.821$

$-55.565$

$-55.928$

$-56.647$.

$-56.511$ 
PRINCI PALE ACTBATE GAAVITY BTATIONB

STA NAME AND NUM

\begin{tabular}{|c|c|}
\hline $\begin{array}{l}13.106 \\
13.112 \\
13.107 \\
13.108 \\
13.109 \\
13.110 \\
13.111 \\
13.201 \\
13.202 \\
13.203 \\
13.204 \\
13.205 \\
13.206 \\
13.315 \\
13.314 \\
13.313 \\
13.312 \\
3.131 \\
33.126 \\
3.127 \\
3.128 \\
13.109 \\
13.301 \\
13.302 \\
13.303 \\
13.304 \\
13.305 \\
13.306 \\
13.318 \\
13.307 \\
13.308 \\
13.309 \\
13.416 \\
13.409 \\
13.408 \\
13.407 \\
13.406 \\
13.405 \\
13.404 \\
13.429\end{array}$ & $\begin{array}{l}734 \\
735 \\
736 \\
737 \\
738 \\
739 \\
740 \\
741 \\
742 \\
743 \\
744 \\
745 \\
746 \\
747 \\
748 \\
749 \\
750 \\
751 \\
752 \\
753 \\
754 \\
1071 \\
755 \\
756 \\
757 \\
758 \\
759 \\
760 \\
761 \\
762 \\
763 \\
764 \\
1070 \\
765 \\
766 \\
767 \\
768 \\
769 \\
770 \\
771\end{array}$ \\
\hline
\end{tabular}

LATITUDE

3841.51

3841.95

38 1 32.28

3843.50

3844.02

$38 \quad 44.71$

$\begin{array}{lll}38 & 43.61\end{array}$

3842.98

3842.43

3842.04

3840.40

3840.91

3836.05

3835.83

3835.11

3834.96

3834.96

$38 \quad 28.33$

$\begin{array}{lll}38 & 29.13\end{array}$

3829.66

38 ( 25.73

$\begin{array}{ll}38 & 30.29 \\ 38 & 30.67\end{array}$

3831.09

3831.88

3832.08

3832.50

3832.02

$38 \quad 32.60$

$38 \quad 32.98$

$38 \quad 31.02$

$\begin{array}{ll}38 & 33.07\end{array}$

$\begin{array}{ll}38 & 33.38 \\ 38 & 33.37\end{array}$

$38 \quad 32.92$

3833.48

$38 \quad 33.89$

3833.70

\section{EAST +}

-81 23.62

$\begin{array}{ll}-81 & 23.24 \\ -81 & 24.66\end{array}$

-81 24.14

$-8123.45$

$\begin{array}{rr}-81 & 22.68 \\ -81 & 22.67\end{array}$

$-8122.31$

$-8121.54$

8120.34

8120.55

8119.44

-8) 23.66

-81 24.46

-81 24.21

$\begin{array}{ll}-81 & 24.21\end{array}$

-81 23.94

-81 23.39

$\begin{array}{ll}-81 & 23.85\end{array}$

$\begin{array}{ll}-81 & 28.28 \\ -81 & 28.52\end{array}$

-81 27.76

$\begin{array}{ll}-81 & 27.09\end{array}$

$\begin{array}{ll}-81 & 25.48 \\ -81 & 24.88\end{array}$

$-8124.67$

-81 24.53

$\begin{array}{ll}-81 & 24.04 \\ -81 & 24.69\end{array}$

$\begin{array}{ll}-81 & 23.38\end{array}$

$\begin{array}{lr}-81 & 8.25 \\ -81 & 20.25\end{array}$

-81 20.12

$-8119.16$

-81 18.04

-81 16.94

$-8116.00$

\section{EVATIO
FEET}

747.000

780.000

97.000

883.000

883.000

795.000

785.000

831.000

910.000

902.000

774.000

740.000

807.000

1120.000

2100.000

1100.000

1100.000

1100.000

673.000

675.000

615.000

714.000

681.000

684.000

706.000

712.000

721.000

748.000

1170.000

1260.000

1260.000

740.000

680.000
670.000

670.000

775.000

722.000

809.000

830.000
TERR COR

MGA

$$
\text { MGNL }
$$

PREE A
MGAL

BOUGUER
MGNL

THEOR
MGAL

T C BOUGUER
MGAL

.350 97952.161

$.400 \quad 979949.607$

.300979946 .210

$.360 \quad 979947.976$

$.190 \quad 979952.961$

$.180 \quad 979954.410$

$.220 \quad 979950.483$

$.270 \quad 979945.420$

$.200 \quad 979945.590$

$.480 \quad 979952.078$

$.440 \quad 979951.438$

$.550 \quad 979950.351$

$.270 \quad 979922.517$

$.480 \quad 979920.719$

97920.721

979920.735

.410 979938.333

$.290 \quad 979938.333$

$0.000 \quad 979935.760$

$.380 \quad 979936.932$

$.270 \quad 979937.007$

.400979937 .410

$.460 \quad 979937.469$

$.490 \quad 979937.476$

$.490 \quad 979935.969$

$.280 \quad 979910.755$

$.290 \quad 979905.545$

$.340 \quad 979905.534$

$0.000 \quad 979946.280$

$.210 \quad 979945.828$

$.190 \quad 979946.998$

$.270 \quad 979946.577$

$.300 \quad 979942.185$

$.190 \quad 979944.361$

$.220 \quad 979939.495$

$.360 \quad 979938.114$
$-56.024980052 .971$

$-57.247980053 .618$

$-56.110 \quad 980054.103$

$-55.933980055 .133$

$-54.982980055 .898$

$-56.038980056 .663$

$-56.171980057 .678$

$-55.755 \quad 980056.060$

$-55.155980055 .133$

$-54.655980054 .324$

$-55.268980053 .750$

$-55.534980051 .339$

$-53.355980052 .089$

-55.278 980044.944

$-57.953 \quad 980014.621$

$-57.334980044 .004$

$-56.672980043 .342$

-56.658980043 .342
-57.499

$-57.499 \quad 980033.605$

$-56.097980034 .779$

$-56.756 \quad 980035.558$

$-57.157 \quad 980029.789$

$-56.743 \quad 980036.483$

$-59.205 \quad 980037.041$

$-59.239 \quad 980037.657$

$-59.144980038 .818$

$-58.955 \quad 980039.111$

$-59.025 \quad 980039.728$

$-57.327 \quad 980038.142$

$-58.122 \quad 980039.023$

$\begin{array}{ll}-58.788 & 980039.875\end{array}$

$\begin{array}{ll}-59.357 & 980040.433\end{array}$

$-51.315 \quad 980041.961$

$-53.969980040 .566$

$-53.854980041 .021$

$-54.260 \quad 980041.006$

$-51.696 \quad 980040.345$

$-53.519 \quad 980041.168$

$\begin{array}{ll}-53.773 & 980041.770 \\ -53.615 & 980041.491\end{array}$
$-53.674$

$-56.847$

$-55.900$

$-55.633$

$-54.622$

$-55.994$

$-55.535$

$-54.885$

$-54.455$

$-54.788$

$-55.094$

$-52.805$

$-55.008$

-57.583
-56.054

256.854

-56.412
-56.338

$-57.139$

$-55.687$

.56.466

$-57.157$

56.363

$-58.935$

$-58.634$

$-58.495$

-58.535

$-58.498$

$-59.017$

51.315

.53.759

.

.990

.396

.

-53.553
-53.255 
PRINCIPAL PACTSAT GRAVITY BTATIONB

STA NAME AND NUM

\begin{tabular}{|c|c|}
\hline $\begin{array}{l}13.428 \\
13.427 \\
13.426 \\
13.425 \\
13.424 \\
13.423 \\
13.424 \\
13.421 \\
13.420 \\
13.419 \\
13.441 \\
13.440 \\
13.439 \\
13.438 \\
13.437 \\
13.446 \\
13.445 \\
13.444 \\
13.442 \\
13.436 \\
13.435 \\
13.434 \\
13.433 \\
13.433 \\
13.432 \\
13.431 \\
13.430 \\
13.402 \\
13.401 \\
13.214 \\
13.213 \\
13.212 \\
13.211 \\
13.2110 \\
13.209 \\
13.208 \\
1.791 \\
1.792 \\
1.793 \\
1.794\end{array}$ & $\begin{array}{l}772 \\
773 \\
774 \\
775 \\
776 \\
777 \\
778 \\
779 \\
780 \\
781 \\
782 \\
783 \\
784 \\
785 \\
786 \\
787 \\
788 \\
789 \\
790 \\
791 \\
792 \\
793 \\
794 \\
795 \\
796 \\
797 \\
798 \\
799 \\
800 \\
801 \\
802 \\
803 \\
804 \\
805 \\
806 \\
807 \\
808 \\
809 \\
810 \\
811\end{array}$ \\
\hline
\end{tabular}

LATITUDE
NORTH +

3832.00

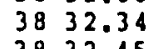
$38 \quad 32.45$ 3831.94 3831.65 3830.69 3830.59 3830.45 3833.61 3834.23 3834.63 3835.12 3837.59 3835.73 3836.73 $38 \quad 36.75$ 3836.83 $\begin{array}{ll}38 & 36.65\end{array}$ 3836.37 3834.62 3834.62 3835.19 3834.73 3833.93 3833.61 $\begin{array}{ll}38 & 36.27 \\ 38 & 37.98\end{array}$ 38.98 3839.55 3839.78 3841.77 $\begin{array}{ll}38 & 40.94 \\ 38 & 39.79\end{array}$ 3834.02 3833.8 3834.95
LONG ITUDE
EAST +

BLEVATIO
FEET

TE

MGAL

MGAL

669.000

750.000

630.000

700.000

635.000

700.000

742.000

742.000

712.000

679.000

679.000

700.000

720.000

778.000

824.000

824.000
1016.000

1140.000

1071.000

869.000

1187.000

1156.000

1156.000

740.000

64.000

693.000

702.000

702.000

830.000

1085.000
789.000

808.000

1068.000

880.000

815.000

840.000

840.000

760.000
900.000

$$
\begin{aligned}
& .2 \\
& .360 \\
& .250 \\
& .3 \\
& .290 \\
& .280 \\
& .3 \\
& .420 \\
& .2 \\
& .36 \\
& .44 \\
& .3 \\
& .350 \\
& .41 \\
& .3 \\
& .43 \\
& .3 \\
& .22 \\
& .1 \\
& .1 \\
& .260 \\
& .2 \\
& .270 \\
& .270 \\
& .3 \\
& .3 \\
& .260 \\
& .450 \\
& .230 \\
& .220 \\
& .170 \\
& .290 \\
& .470 \\
& .350 \\
& .4 \\
& .430 \\
& .490 \\
& .280 \\
& .290 \\
& .3 \\
&
\end{aligned}
$$

$\begin{array}{ll}.240 & 979948.477 \\ .360 & 979947.539\end{array}$

$.250 \quad 979940.222$

979948.482

979944.488

979947.271

979946.373
979939.397

$210 \quad 979939.390$

360979939.838

$.440 \quad 979946.685$

$.390 \quad 979946.559$

$350 \quad 979946.528$

$.410 \quad 979945.568$

.979943 .306

$.320 \quad 979940.684$

$.210 \quad 979930.910$

$.180 \quad 979922.615$

$.180 \quad 979931.128$

$260 \quad 979944.163$

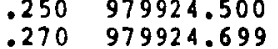
979924.699
979924.699 979938.508 979950.512 979951.715 979952.520 979953.257 979948.857 979934.155 979949.197 979952.239 979940.565 979940.565
979950.748 979552.232 979952.232 979938.207 979938.207 979941.725
979938.289

REE AI
MGNL

$-27.590$ $-30.438$ -28.887
-31.797 $-28.57$ -28.574
-31.480 $-26.70$ $-27.885$ $-27.885$ $-29.907$ $-34.474$ $-31.843$ $-30.487$ $-30.285$ $-30.722$ $-28.539$ $-27.003$ $-16.496$ $-16.245$ $-13.950$ $-19.512$ 0.408 $-9.408$ $-24.750$ $-22.887$ $-24.930$ $-24.689$ $-25.979$ $-20.852$ $-12.378$ $-26.677$ $-22.186$ $-12.331$ $-18.611$ $-24.549$ $-24.743$ $-24.537$ -29.411
-20.383

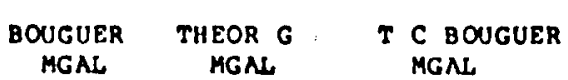

$-50.407 \quad 980038.994$ $-50.167$ $-52.745 \quad 980039.493$ $-54.467 \quad 980039.655 \quad-52.385$ $-53.285 \quad 980039.537 \quad-52.905$
-52.449 $-52.449 \quad 980038.906 \quad-52.159$
-53.1238 $-53.138 \quad 980038.480 \quad-52.858$ $-50.579 \quad 980038.920 \quad-50.269$ $-53.193 \quad 980037.070 \quad-52.773$ $-53.047 \quad 980036.923 \quad-52.837$ $-54.192 \quad 980036.718 \quad-53.832$ $\begin{array}{ll}-56.303 & 980041.359 \quad-55.863\end{array}$ $-55.002 \quad 980042.270 \quad-54.612$ $-54.362 \quad 980042.858 \quad-54.012$
$-54.842 \quad 980043.577 \quad-54.432$ $\begin{array}{lll}-54.842 & 980043.577 \quad-54.432\end{array}$ $\begin{array}{lll}-57.257 & 980047.208 & -56.867\end{array}$ $\begin{array}{lll}-55.825 & 980044.474 \quad-55.395\end{array}$ $\begin{array}{lll}-55.108 & 980045.194 \quad-54.788\end{array}$ $\begin{array}{lll}-54.149 & 980045.973 & -53.939\end{array}$ $\begin{array}{lll}-55.128 & 980046.090 \quad-54.948\end{array}$ $\begin{array}{lll}-50.486 & 980045.826 & -50.306\end{array}$ $-49.152 \quad 980045.414 \quad-48.892$
-450.468 $\begin{array}{lll}-50.468 & 980046.135 \quad-50.218\end{array}$ $-48.836 \quad 980042.843 \quad-48.566$ $-48.836 \quad 98042.843 \quad-48.566$ $-48.126 \quad 900043.004 \quad-47.736$ $-48.5660900041 .8290-48.306$ $-47.951 \quad 980041.359-47.501$ $-49.923 \quad 900045.267-49.693$ $-49.161 \quad 980047.781 \quad-48.941$ $-49.384 \quad 980048.589 \quad-49.214$ $-53.588 \quad 980050.089 \quad-53.298$ $-49.745 \quad 980050.427 \quad-49.275$ $-48.757 \quad 980053.353 \quad-48.407$ $-48.625 \quad 980052.133-048.145$ $-49.347 \quad 980050.442-48.917$ $-53.393 \quad 980041.961-52.903$ $-53.187 \quad 980041.756 \quad-52.907$ $\begin{array}{lll}-55.333 & 980042.622 & -55.043\end{array}$ $\begin{array}{lll}-51.079 & 980043.328 & -50.709\end{array}$ 
PRINCIPAL PACTSAT GRAVITY.STATIONA

GTA NAME AND NUM

1.795
1.796
1.797
1.798
1.791
1.791
7.910
7.911
7.912
7.913
7.914
7.915
7.916
7.917
7.918
7.919
7.920
7.921
7.922
7.923
7.921
7.925
7.926
7.927
7.928
7.929
7.930
7.402
7.401
7.213
7.212
7.211
7.226
7.227
7.228
7.229
7.230
7.203
7.204
7.219
LATITUDE
NORTH +

3835.09

3835.05

3834.72

3834.36

3834.02

3835.28

3835.75

3835.75

38.36 .39

3837.00

$38 \quad 37.15$

$38 \quad 37.10$

$38 \quad 37.02$

3837.35

$38 \quad 37.15$

$\begin{array}{ll}38 & 37.24 \\ 38 & 37.17\end{array}$

$38 \quad 37.05$

3836.69

$38 \quad 36.62$

3836.60

3836.79

3836.92

3835.95

3836.75

$38 \quad 36.76$

3838.40

3839.0

3839.00

3839.17

3839.78

$\begin{array}{lll}38 & 40.45\end{array}$

3841.1

$\begin{array}{rr}38 & 41.59 \\ 38 & 41.35\end{array}$

$\begin{array}{cc}\text { LONGITUDE } \\ \text { EAST + } \\ -81 & 11.50 \\ -81 & 11.13 \\ -81 & 13.43 \\ -81 & 15.00 \\ -81 & 8.25 \\ -81 & 8.25 \\ -81 & 15.67 \\ -81 & 4.57 \\ -81 & 2.72 \\ -81 & 1.68 \\ -81 & 1.12 \\ -81 & .35 \\ -80 & 59.31 \\ -80 & 58.32 \\ -80 & 57.25 \\ -80 & 56.21 \\ -80 & 55.25 \\ -80 & 54.20 \\ -80 & 52.95 \\ -80 & 52.17 \\ -80 & 51.12 \\ -80 & 50.21 \\ -80 & 49.11 \\ -80 & 47.99 \\ -80 & 39.49 \\ -80 & 39.11 \\ -80 & 39.20 \\ -81 & 2.40 \\ -81 & 2.37 \\ -81 & 2.62 \\ -81 & 3.02 \\ -81 & 4.21 \\ -81 & 4.79 \\ -81 & 5.57 \\ -81 & 6.15 \\ -81 & 5.50 \\ -81 & 5.89 \\ -81 & 6.13 \\ -81 & 5.50 \\ -81 & 3.93 \\ & \\ & \end{array}$

ELEVTION
FEET

TERR COR
MGAL

MGAL

900.000

709.000

876.000

740.000

740.000

980.000

920.000

860.000

8.80 .000

902.000

983.000

1140.000

1160.000

1138.000

1117.000

1138.000

1140.000

1000.000

900.000

1075.000

1077.000

1028.000

1490.000

1380.000

1400.000

860.000

959.000

967.000

907.000

846.000

849.000

880.000

960.000

880.000

850.000

850.000

802.000

868.000

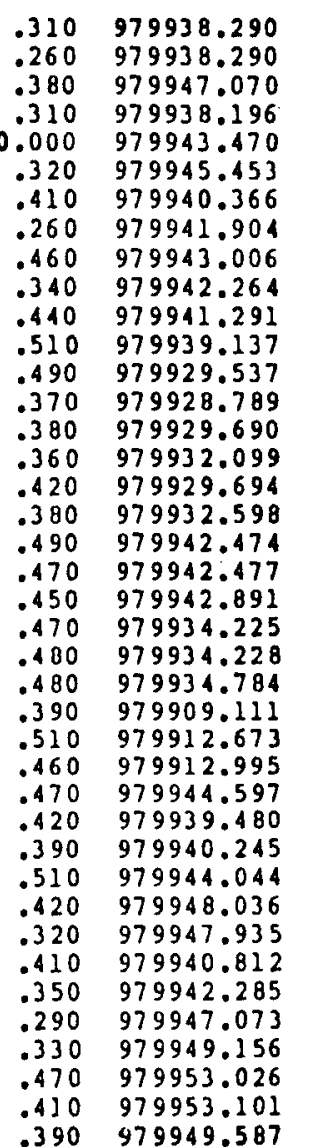

PREE A
MGAL

BOUGUER
MGAL

THEOR G
HGAL

C BOUGUER
MGNL

$-20.588$

$-20.529$

$-29.230$

$-28.886$

$-26.903$

$-11.267$

$-20.604$

$-20.406$

$-20.206$

$-15.255$

$-9.794$

$-8.587$

$-9.638$

$-9.204$

$-10.118$

$-6.733$

$-10.157$

$-10.052$

$-18.867$

$-10.543$

$-10.250$

$-14.273$

3.230

$-3.745$

$-1.438$

$-16.288$

$-14.785$

$-19.040$

$-22.036$

$-21.517$

$-25.694$

$-16.946$

$-20.580$

$-22.304$

$-24.037$

$-23.516$
$-51.285 \quad 980043.533$

$-51.225980043 .475$

$-50.975$

$\begin{array}{lll}-51.745 & 980042.461 & -51.435\end{array}$

$\begin{array}{lll}-54.125 & 980041.961 & -54.125\end{array}$

$\begin{array}{lll}-52.142 & 980041.961 & -51.822\end{array}$

$\begin{array}{lll}-41.692 & 980013.813 \quad-44.282\end{array}$

$\begin{array}{lll}-47.441 & 980044.503 \quad-47.181\end{array}$

$\begin{array}{lll}-49.936 & 980044.503 & -49.476\end{array}$

$\begin{array}{lll}-50.420 & 980045.444 & -50.080\end{array}$

$\begin{array}{lll}-48.783 & 980046.855 & -48.27\end{array}$

$\begin{array}{lll}-48.676 & 980046.561 & -48.186\end{array}$

$\begin{array}{lll}-48.151 & 980046.487 & -47.781\end{array}$

$\begin{array}{lll}48.452 & 980046.370 \quad-48.072\end{array}$

$-47.302 \quad 900046.370 \quad-16.942$
-48.932

$\begin{array}{lll}-48.932 & 980046.855 & -48.512\end{array}$

$\begin{array}{lll}-45.615 & 980046.561 & -45.235\end{array}$

$\begin{array}{lll}-44.159 & 980046.590 & -43.689\end{array}$

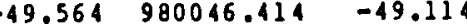

$\begin{array}{lll}-47.208 & 980045.885 & -46.738\end{array}$

$\begin{array}{lll}-46.983 & 980045.782 & -46.503\end{array}$

$\begin{array}{lll}-49.335 & 980045.752 & -48.855\end{array}$

$\begin{array}{lll}-47.589 & 980046.032 & -47.199\end{array}$

$\begin{array}{lll}-50.813 & 980046.223 & -50.30\end{array}$

$\begin{array}{lll}-49.188 & 980046.120 & -48.728\end{array}$

$\begin{array}{lll}-40.639 & 980044.797 & -48.169\end{array}$

$\begin{array}{lll}-48.997 & 980045.973 & -48.577\end{array}$

$\begin{array}{llll}-49.975 & 980048.398 & -49.465\end{array}$

$-50.47$

$\begin{array}{llll}-50.474 & 980049.310 & -50.154\end{array}$

$\begin{array}{lll}-55.708 & 980049.280 & -55.298\end{array}$

$\begin{array}{lll}-49.689 & 980049.530 & -49.339\end{array}$

$-50.595,980050.427 \quad-50.305$

$\begin{array}{lll}-51.295 & 980051.112 & -50.965\end{array}$

$\begin{array}{lll}-51.391 & 980052.500 & -50.921\end{array}$

$\begin{array}{rrr}-51.245 & 980053.089 & -50.835 \\ -51.109 & 980052.736 & -50.719\end{array}$ $\begin{array}{lll}-50.970 & 980046.340 \quad-50.530\end{array}$

$\begin{array}{lll}-44.264 & 980046.693 & -43.774\end{array}$

$\begin{array}{lll}-47.767 & 980045.988 & -47.377\end{array}$ 
PRINCIPAL PACTSATG GAVITY STATION

STA NAME AND NUM

7.220
7.221
7.222
7.223
7.224
7.217
7.218
7.216
7.234
7.215
7.236
7.235
7.233
7.214
7.208
7.207
7.232
7.206
7.205
7.202
7.201
7.204
7.103
7.102
7.122
7.123
7.124
7.125
7.126
7.209
7.210
7.403
7.404
7.405
7.006
7.407
7.408
7.409
7.301
7.326
LATITUDE

$\begin{array}{lll}38 & 41.31 \\ 38 & 41.29\end{array}$

38 3 41.29

38042.0

38 3 31.49

38 30.63

$\begin{array}{lll}38 & 43.78\end{array}$

$\begin{array}{ll}38 & 44.24 \\ 38 & 43.98\end{array}$

$\begin{array}{lll}38 & 43.08\end{array}$

3842.54

$\begin{array}{ll}38 & 44.04 \\ 38 & 44.73\end{array}$

$\begin{array}{lll}38 & 44.91\end{array}$

$\begin{array}{lll}38 & 43.77\end{array}$

$\begin{array}{ll}38 & 41.88 \\ 38 & 41.67\end{array}$

3841.14

$\begin{array}{lll}38 & 40.55\end{array}$

3839.94

3839.89

$\begin{array}{ll}38 & 39.38 \\ 38 & 38.87\end{array}$

$\begin{array}{lll}38 & 40.42\end{array}$

$\begin{array}{ll}38 & 41.18 \\ 38 & 41.83\end{array}$

3842.69

3843.33

$\begin{array}{ll}38 & 41.06 \\ 38 & 40.35\end{array}$

835.15

38.52

3831.94

$38 \quad 31.17$

3830.04

3831.65

$\begin{array}{ll}38 & 30.55 \\ 38 & 31.56\end{array}$

\section{EAST +}

$-81 \quad 3.48$

$\begin{array}{ll}-81 & 3.48 \\ -81 & 2.80\end{array}$

-81 2.39

$-81 \quad 1.44$

-81 1.36

-81 ..14

$-81 \quad 1.32$

-81 2.16

$\begin{array}{ll}-81 & 2.69 \\ -81 & 2.77\end{array}$

$\begin{array}{ll}-81 & 3.25\end{array}$

$-81 \quad 3.92$

$\begin{array}{ll}-81 & 5.15\end{array}$

$\begin{array}{ll}-81 & 5.98 \\ -81 & 6.35\end{array}$

$\begin{array}{ll}-81 & 6.35 \\ -81 & 6.32\end{array}$

$-815.87$

$\begin{array}{ll}-81 & 5.40\end{array}$

$\begin{array}{ll}-81 & 6.89\end{array}$

$\begin{array}{ll}-81 & 8.11\end{array}$

$\begin{array}{lll}-81 & 8.21\end{array}$

$\begin{array}{ll}-81 & 7.90\end{array}$

$\begin{array}{ll}-81 & 8.57 \\ -81 & 8.60\end{array}$

$\begin{array}{ll}-81 & 8.60 \\ -81 & 8.47\end{array}$

$-81 \quad 9.02$

$\begin{array}{ll}-81 & 8.65 \\ -81 & 4.89\end{array}$

$\begin{array}{ll}-81 & 4.89\end{array}$

$\begin{array}{ll}-81 & 4.67 \\ -81 & 2.45\end{array}$

$-81 \quad 1.40$

$\begin{array}{ll}-81 & 1.67 \\ -81 & 1.75\end{array}$

$-81 \quad 1.62$

$-813.86$

$\begin{array}{ll}-81 & 3.86 \\ -81 & 4.56\end{array}$

$\begin{array}{ll}-81 & 7.72 \\ -81 & 8.85\end{array}$

\section{LEVATION}

858.000 883.000 .000 89.000 926.000 85.000 920.000 816.000 806.000 792.000 906.000 957.000 860.000 767.000 767.000 766.000 774.000 787.000 795.000 846.000

1145.000

863.000

877.000

865.000

26.000

814.000

805.000

804.000

810.000

48.000

909.000

799.000

880.000

730.000

892.000

845.000
2126.000 MGAL MLAC

MGN

OUGUER

HEOR

C BOUGUER

$.550 \quad 979951.185$

-20.787
-20.941

$-50.051 \quad 980052.677$ $-51.057 \quad 980052.647$ $-51.2359800$ $-51.18498053 .69$ $-51.140 \quad 90052.94$ -50.692 g80051.083 80051.677 980056.310 $-51.833 \quad 98005$ $-51.672980056 .604$ $-49.428 \quad 980055.280$
-51.948 $-51.948980054 .486$ -52.500 900056.092 -52.058 980057.708 $-51.337980057 .972$ $-53.244980056 .295$ $-51.253980053 .515$ -50.019 980053.206 $-50.18090052 .427$ $-51.767980050 .662$ $-51.571 \quad 980050.589$ -51.328 980051.368 $-51.926980052 .486$ $-52.235980053 .442$ $-52.114900054 .707$ -52.353 .980055.648 52.353 980055.648 $-51.370 \quad 980051.265$ $-48.052980043 .622$ $-48.520 \quad 980042.696$ $-48.340 \quad 900040.771$ $-47.53790038 .906$ $-45.920 \quad 980037.775$ -47.308 98003. $-47.308980038 .480$ $\begin{array}{ll}-47.247 & 980036.864 \\ -50.086 & 980038.348\end{array}$
$-49.501$ $-50.567$ $-50.594$ $-50.594$ $-50.670$ $-50.603$ $-50.032$ $-52.289$ $-51.333$ $-51.162$ $-51.458$ $-51.458$ $-51.968$ $-50.747$ $-52.634$ $-50.483$ $-49.500$ $-50.944$ $-51.177$ $-50.700$ $-50.958$ $-51.516$ -51.765 $-51.643$ $-50.900$ $-50.700$ $-47.802$ $-48.250$ $-48.040$ $-47.257$ $-45.680$ $-47.371$ $-46.958$ $-49.876$ 
PRINCIPAL P.ACTSAT GRAVITY BTATIONS

STA NAME AND NUM

7.325
7.327
7.111
7.302
7.303
7.304
7.399
7.305
7.306
7.307
7.308
7.309
7.310
7.313
7.311
7.305
7.106
7.107
7.108
7.109
7.110
7.111
7.112
7.113
7.114
7.116
7.117
7.118
7.119
7.120
7.121

NATITUDE

EAST +

8118.86

3831.95

3831.95

3831.66

3834.26

3834.01

3835.15

3835.48

3836.05

$38 \quad 36.56$

3836.71

$38 \quad 37.16$

$38 \quad 37.55$

3839.02

$38 \quad 39.86$

3840.75

$38 \quad 41.45$

3841.77

3842.62

$38 \quad 43.04$

3843.14

$38 \quad 42.39$

$\begin{array}{ll}38 & 42.23 \\ 38 & 43.94\end{array}$

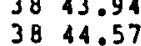

18.86

$-817.14$

-81 7.97

$-817.85$

-81 8.41

-81
-81 $\quad 9.69$

$\begin{array}{ll}-81 & 10.46 \\ -81 & 10.78\end{array}$

$-8111.91$

$\begin{array}{ll}-81 & 11.99 \\ -81 & 12.31\end{array}$

$-8112.82$

$\begin{array}{ll}-81 & 14.69 \\ -81 & 14.72\end{array}$

-81
-81
-814.72

$\begin{array}{ll}-81 & 14.08 \\ -81 & 23.39\end{array}$

$\begin{array}{ll}-81 & 13.01 \\ -81 & 12.20\end{array}$

$-8112.13$

$-8111.82$

$-8111.87$

$\begin{array}{ll}-81 & 11.74 \\ -81 & 12.03\end{array}$

$\begin{array}{lll}-81 & 12.02\end{array}$

$-8112.22$

$-8113.4$

$-8124.48$

$\begin{array}{lll}-81 & 12.43 \\ -81 & 13.31\end{array}$
ELEVATIO

FEET

1285.000

1285.000

1020.000

790.000

765.000

749.000

774.000

721.000

717.000

736.000

764.000

804.000

729.000

739.000

773.000

820.000

934.000

845.000

812.000

811.000

800.000

778.000

774.000

771.000

809.000

864.000

754.000

748.000

:

MGN

MGAL

MGAL

BOUGUER
MGAL

THEOR

MGAL

T C BOUGUER

MGAL

$\begin{array}{lll}.190 & 979913.432 & -4.619\end{array}$

-4.619
-12.771

$-48.147 \quad 980038.920$

980038.920

$-47.560 \quad 980038.495$

$-51.225980054 .324$

980042.314

$-21.943$

$-23.926$

980041.94

980042.637

980043.622

980043.813

980044.944

980045.694

980046.443

980045.914

980046.575

980047.149

980047.825

980049.310

900050.545

980051.603

980051.853

980052.883

980053.353

980053.97

980054.604

980055.22

980055.368

980054.265

980054.030

980056.545

980057.472
$-48.257$

$-48.156$

$-50.845$

$-48.677$

$-49.618$

$-49.043$

$-50.150$

-49.743
-51.214

-51.214
-51.057

$-51.867$

$-52.610$

$-52.430$

$-51.751$

$-52.121$

$-52.083$

-51.970
-51.197

$-51.220$

$-51.893$

$-51.883$

$-52.107$

$-52.281$

$-52.301$

52.110

-52.345
-52.513 


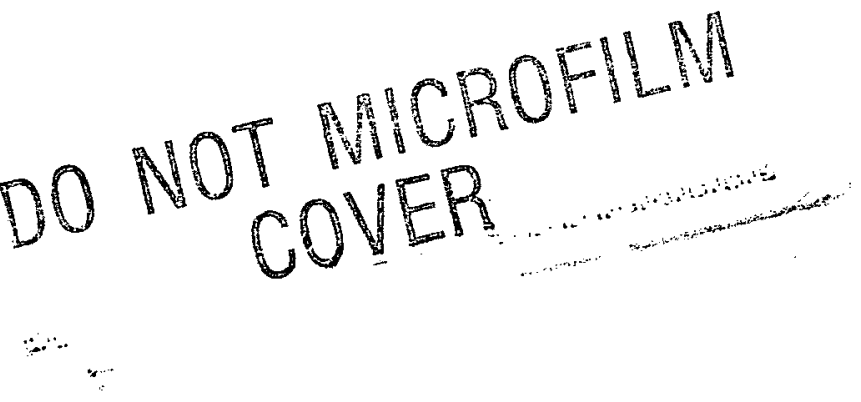

Printed in the -United States of America Available from

National Technical Information Service

US Department of Commerce

5285 Port Royal Road

Springfield, VA 22161

Microfiche (A01)

\begin{tabular}{cc} 
Page Range & $\begin{array}{c}\text { NTIS } \\
\text { Price Code }\end{array}$ \\
\hline $001-025$ & A02 \\
$026-050$ & A03 \\
$051-075$ & A04 \\
$076-100$ & A05 \\
$101-125$ & A06 \\
$126-150$ & A07
\end{tabular}

\begin{tabular}{c:c} 
Page Range & $\begin{array}{c}\text { NTIS } \\
\text { Price Code }\end{array}$ \\
\hline $151-175$ & A08 \\
$176-200$ & A09 \\
$201-225$ & A 10 \\
$226-250$ & A11 \\
$251-275$ & A12 \\
$276-300$ & A13
\end{tabular}

\begin{tabular}{cc} 
Page Range & $\begin{array}{c}\text { NTIS } \\
\text { Price Code }\end{array}$ \\
\hline $301-325$ & A 14 \\
$326-350$ & A 15 \\
$351-375$ & A 16 \\
$376-400$ & A 17 \\
$401-425$ & A18 \\
$426-450$ & A19
\end{tabular}

\begin{tabular}{cc} 
Page Range & $\begin{array}{c}\text { NTIS } \\
\text { Price Code }\end{array}$ \\
\hline $451-475$ & A20 \\
$476-500$ & A21 \\
$501-525$ & A22 \\
$526-550$ & A23 \\
$551-575$ & A24 \\
$576-600$ & A25 \\
$601-$ up $^{*}$ & A99
\end{tabular}

${ }^{*}$ Contact NTIS for a price quote. 
and

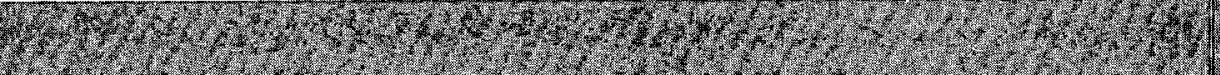

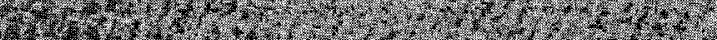

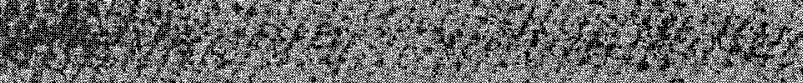

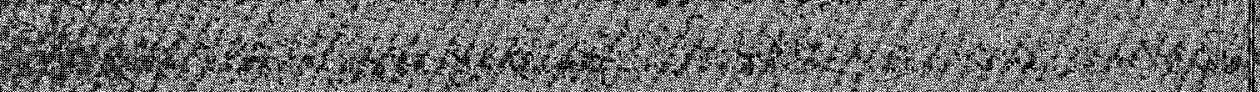 (2) 2. 1.}

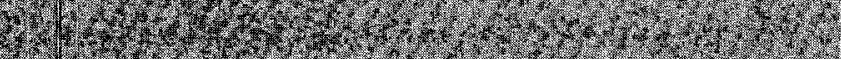

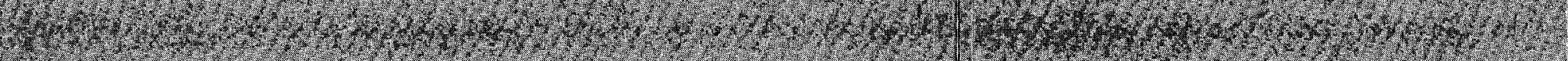

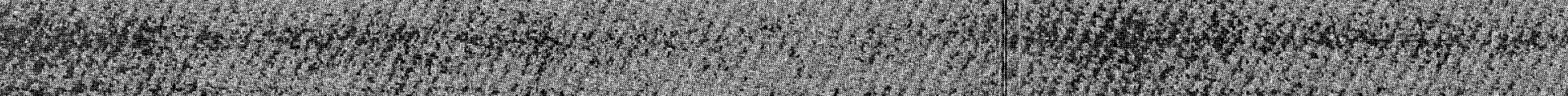

20.5 (6)

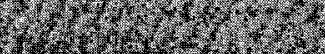

H.t.

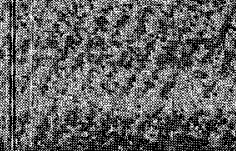

H

H.

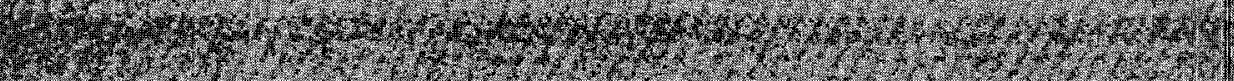

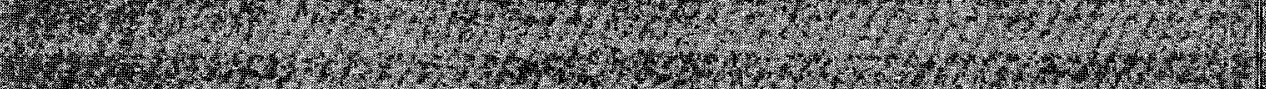

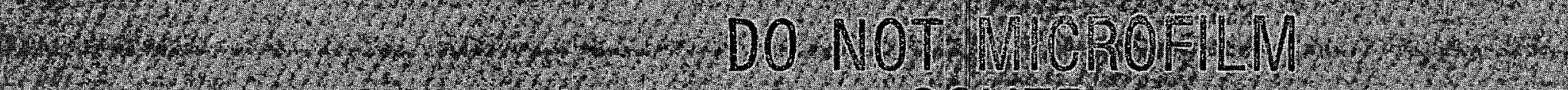

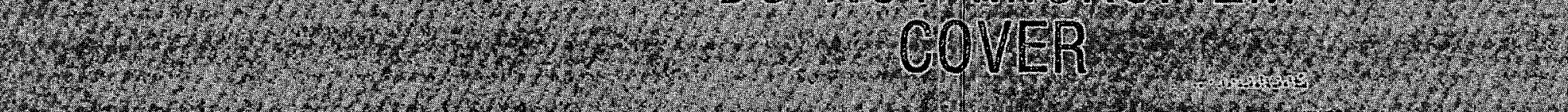
H.

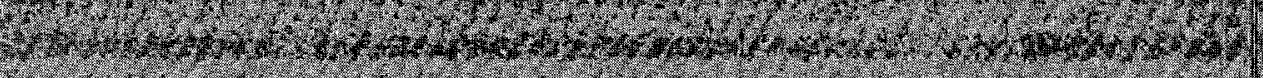
(1.

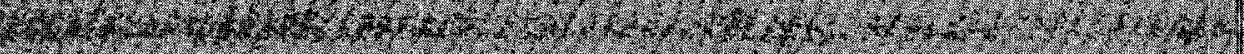
1.

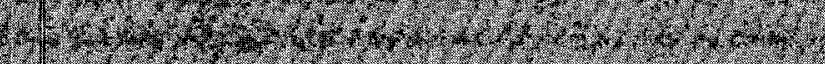

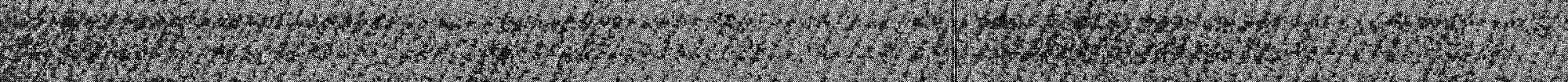
(5)

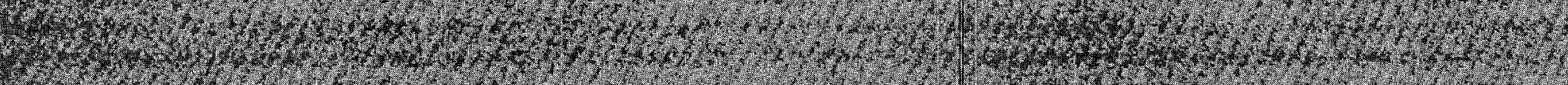

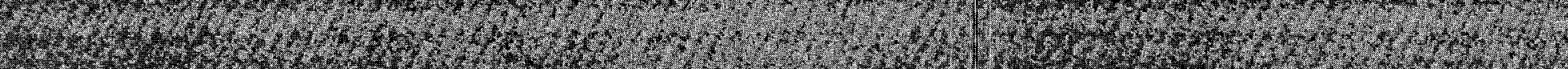

\section{Los Alamos}

